\title{
The Hamiltonian structure of the nonlinear Schrödinger equation and the asymptotic stability of its ground states
}

\author{
Scipio Cuccagna
}

\author{
8.1 .11
}

\begin{abstract}
In this paper we prove that ground states of the NLS which satisfy the sufficient conditions for orbital stability of M.Weinstein, are also asymptotically stable, for seemingly generic equations. Here we assume that the NLS has a smooth short range nonlinearity. We assume also the presence of a very short range and smooth linear potential, to avoid translation invariance. The basic idea is to perform a Birkhoff normal form argument on the hamiltonian, as in a paper by Bambusi and Cuccagna on the stability of the 0 solution for NLKG. But in our case, the natural coordinates arising from the linearization are not canonical. So we need also to apply the Darboux Theorem. With some care though, in order not to destroy some nice features of the initial hamiltonian.
\end{abstract}

\section{Introduction}

We consider the nonlinear Schrödinger equation (NLS)

$$
\mathrm{i} u_{t}=-\Delta u+V u+\beta\left(|u|^{2}\right) u, u(0, x)=u_{0}(x),(t, x) \in \mathbb{R} \times \mathbb{R}^{3}
$$

with $-\Delta+V(x)$ a selfadjoint Schrödinger operator. Here $V(x) \neq 0$ to exclude translation invariance. We assume that both $V(x)$ and $\beta\left(|u|^{2}\right) u$ are short range and smooth. We assume that (1.1) has a smooth family of ground states. We then prove that the sufficient conditions for orbital stability by Weinstein [W1] (which, essentially, represent the correct definition of linear stability, see [Cu3]), imply for a generic (1.1) that the ground states are not only orbitally stable, as proved in [W1] (under less restrictive hypotheses), but that their orbits are also asymptotically stable. That is, a solution $u(t)$ of (1.1) starting sufficiently close to ground states, is asymptotically of the form $e^{i \theta(t)} \phi_{\omega_{+}}(x)+e^{i t \Delta} h_{+}$, for $\omega_{+}$a fixed number and for $h_{+} \in H^{1}\left(\mathbb{R}^{3}\right)$ a small energy function. The problem of stability of ground states has a long history. Orbital stability has been well understood since the 80's, see in the sequence [CL, W1, GSS1, GSS2], and 
has been a very active field afterwards. Asymptotic stability is a more recent, and less explored, field. In the context of the NLS the first results are in the pioneering works [SW1, SW2, BP1, BP2]. Almost all references on asymptotic stability of ground states of the NLS tackle the problem by first linearizing at ground states, and by attempting to deal with the resulting nonlinear problem for the error term. An apparent problem in the linear theory is that the linearization is a not symmetric operator. However the linearization is covered by the scattering theory of non selfadjoint operators developed by T.Kato in the 60 's, see his classical [K], see also [CPV, S]. Dispersive and Strichartz estimates for the linearization, analogous to the theory for short range scalar Schrödinger operators elaborated in [JSS, Y1, Y2], to name only few of many papers, can be proved using similar ideas, see for example [Cu1, S, KS]. It is fair to say that anything that can be proved for short range scalar Schrödinger operators, can also be proved for the linearizations. The only notable exception is the problem of "positive signature" embedded eigenvalues, see [Cu3], which we conjecture not to exist (in analogy to the absence of embedded eigenvalues for short range Schrödinger operators), and which in any case are unstable, see [CPV]. Hence it is reasonable to focus on NLS's where these positive signature embedded eigenvalues do not exist (in the case of ground states, all positive eigenvalues are of positive signature). While linear theory is not a problem in understanding asymptotic stability, the real trouble lies in the difficult NLS like equation one obtains for the error term. Specifically, the linearization has discrete spectrum which, at the level of linear theory, tends not to decay and potentially could yield quasiperiodic solutions. A good analogy with more standard problems, is that the continuous spectrum of the linearization corresponds to stable spectrum while the discrete spectrum corresponds to central directions. Stability cannot be established by linear theory alone. The first intuition on how nonlinear interactions are responsible for loss of energy of the discrete modes, is in a paper by Sigal [Si]. His ideas, inspired by the classical Fermi golden rule in linear theory, are later elaborated in [SW3], to study asymptotic stability of vacuum for the nonlinear Klein Gordon equations with a potential with non empty discrete spectrum. This problem, easier than the one treated in the present paper, to a large extent is solved in [BC]. In reality, the main ideas in [SW3] had already be sketched, for the problem of stability of ground states of NLS, in a deep paper by Buslaev and Perelman [BP2], see also the expanded version [BS]. In the case when the linearization has just one positive eigenvalue close to the continue spectrum, [SW3, BP2], or [Si] in a different context, identify the mechanisms for loss of energy of the discrete modes in the nonlinear coupling of continuous and discrete spectral components. Specifically, in the discrete mode equation there is a key coefficient of the form $\langle D F, F\rangle$ for $D$ a positive operator and $F$ a function. Assuming the generic condition $\langle D F, F\rangle \neq 0$, this gives rise to dissipative effects leading to leaking of energy from the discrete mode to the continuous modes, where energy disperses because of linear dispersion, and to the ground state. After [BP2] there is strong evidence that, generically, linearly stable ground states, in the sense of [W1], should be asymptotically stable. Still, it is a seemingly technically difficult problem to solve rigorously. 
After [BP2, SW3], a number of papers analyze the same ideas in various situations, [TY1, TY2, TY3, T, SW4, Cu2]. In the meantime, a useful series of papers [GNT, M1, M2] shows how to use endpoint Strichartz and smoothing estimates to prove in energy space the result of [SW2, PiW], generalizing the result and simplifying the argument. The next important breakthrough is due to Zhou and Sigal [GS]. They tackle for the first time the case of one positive eigenvalue arbitrarily close to 0, developing further the normal forms analysis of [BP2] and obtaining the rate of leaking conjectured in [SW3] p.69. The argument is improved in $[\mathrm{CM}]$. The crucial coefficient is now of the form $\langle D F, G\rangle$, with $F$ and $G$ not obviously related. In $[\mathrm{CM}]$ it is noticed that $\langle D F, G\rangle<0$ is incompatible with orbital stability (an argument along these lines is suggested in [SW3] p.69). So, for orbitally stable ground states, the generic condition $\langle D F, G\rangle \neq 0$ implies positivity, and hence leaking of energy out of the discrete modes. This yields a result similar to [Si, BP2, SW3] and in particular is a partially positive answer to a conjecture on p.69 in [SW3]. The case with more than one positive eigenvalue is harder. In this case, due to possible cancelations, $[\mathrm{CM}]$ is not able to draw conclusions on the sign of the coefficients under the assumption of orbital stability. But, apart from the issue of positivity of the coefficients, $[\mathrm{CM}]$ shows that the rest of the proof does not depend on the number of positive eigenvalues. Moreover, [T, GW1, Cu3] show that if there are many positive eigenvalues, all close to the continuous spectrum, then the important coefficients are again of the form $\langle D F, F\rangle$. The reason for this lies in the hamiltonian nature of the NLS. The above papers contain normal forms arguments. The hamiltonian structure is somewhat lost in the above papers. When the eigenvalues are close to the continuous spectrum, the normal form argument consists of just one step. This single step does not change the crucial coefficients. Then, the hamiltonian nature of the initial system, yields information on these coefficients (this is emphasized in [Cu3]). In the case treated in $[\mathrm{GS}, \mathrm{CM}]$ though, there are many steps in the normal form. The important coefficients are changed in ways which look very complicated, see $[\mathrm{Gz}]$ which deals with the next two easiest cases after the easiest. The correct way to look at this problem is introduced in $[\mathrm{BC}]$, which deals with the problem introduced in [SW3]. Basically, the positivity can be seen by doing the normal form directly on the hamiltonian. We give a preliminary and heuristic justification on why the hamiltonian structure is crucial at the end of section 3. $[\mathrm{BC}]$ consists in a mixture of a Birkhoff normal forms argument, with the arguments in $[\mathrm{CM}]$. For asymptotic stability of ground states of NLS though, $[\mathrm{BC}]$ is still not enough. Indeed in $[\mathrm{BC}]$ something peculiar happens: the natural coordinates arising by the spectral decomposition of the linearization at the vacuum solution, are also canonical coordinates for the symplectic structure. This is no longer true if instead of vacuum we consider ground states. So we need an extra step, which consists in the search of canonical coordinates, through the Darboux theorem. This step requires care, because we must make sure that our problem remains similar to a semilinear NLS also in the new system of coordinates.

In a forthcoming paper, Zhou and Weinstein [GW2] track precisely in the setting of [GW1] how much of the energy of the discrete modes goes to the 
ground state and how much is dispersed. For another result on asymptotic stability, that is asymptotic stability of the blow up profile, we refer to [MR]. In some respects the situation in $[\mathrm{MR}]$ is harder than here, since there the additional discrete modes are concentrated in the kernel of the linearization. There is important work on asymptotic stability for $\mathrm{KdV}$ equations due to Martel and Merle, see [MM1] and further references therein, which solve a problem initiated by Pego and Weinstein [PW], the latter closer in spirit to our approach to NLS. It is an interesting question to see if elaboration of ideas in [MM1, MMT] can be used for alternative solutions of the problem which we consider here. Our result does not cover important cases, like the pure power NLS, with $\beta\left(|u|^{2}\right)=-|u|^{p-1}$ and $V=0$, where our result is probably false. Indeed it is well known that in $3 \mathrm{D}$ ground states are stable for $p<7 / 3$ and unstable for $p \geq 7 / 3$. In the $p<7 / 3$ case there are ground states of arbitrarily small $H^{1}$ norm. They are counterexamples to the asymptotic stability in $H^{1}$ of the 0 solution. Then for $p>5 / 3$ the 0 solution is asymptotically stabile in a smaller space usually denoted by $\Sigma$, which involves also the $\|x u\|_{L_{x}^{2}}$ norm, see in [St] the comments after Theorem 6 p. 55. In $\Sigma$ there are no small ground states for $p \in(5 / 3,7 / 3)$. Presumably one should be able to prove asymptotic stability of ground states in $\Sigma$. To our knowledge even the following (presumably easier) problem is not solved yet: the asymptotic stability of 0 in $\Sigma$ when $V \neq 0, \sigma_{p}(-\Delta+V)=\emptyset$ and $\beta\left(|u|^{2}\right)=-|u|^{p-1}$ with $p \in(5 / 3,7 / 3)$. In the literature on asymptotic stability of ground states like [BP2, BS, GS, CM], the case of moving solitons is left aside, because in that set up it appears substantially more complex. We do not treat moving solitons here either, but it is possible that our approach might help also with moving solitons. In the step when we perform the Darboux Theorem, the velocity should freeze and we should reduce to the same situation considered from section 8 on. The extra difficulty with moving solitons is that there are more obstructions to the fact that after Darboux we have a semilinear NLS. But it would be surprising if this difficulty had a really deep nature. In any case, the main conceptual problem stemming from [Si, BP2, SW3], which we solve here, is the issue of the positive semidefiniteness of the critical coefficients. There is a growing literature on interaction between solitons, see for example [MM2, HW, M3], and we expect our result to be relevant.

We do not reference all the literature on asymptotic stability of ground states, see $[\mathrm{CT}]$ for more. We like to conclude observing that Sigal [Si], Buslaev and Perelman [BP2] and Soffer and Weinstein [SW3] had identified with great precision the right mechanism of leaking of energy away from the discrete modes.

\section{Statement of the main result}

We will assume the following hypotheses.

(H1) $\beta(0)=0, \beta \in C^{\infty}(\mathbb{R}, \mathbb{R})$. 
(H2) There exists a $p \in(1,5)$ such that for every $k \geq 0$ there is a fixed $C_{k}$ with

$$
\left|\frac{d^{k}}{d v^{k}} \beta\left(v^{2}\right)\right| \leq C_{k}|v|^{p-k-1} \quad \text { if }|v| \geq 1 .
$$

(H3) $V(x)$ is smooth and for any multi index $\alpha$ there are $C_{\alpha}>0$ and $a_{\alpha}>0$ such that $\left|\partial_{x}^{\alpha} V(x)\right| \leq C_{\alpha} e^{-a_{\alpha}|x|}$.

(H4) There exists an open interval $\mathcal{O}$ such that

$$
\Delta u-V u-\omega u+\beta\left(|u|^{2}\right) u=0 \quad \text { for } x \in \mathbb{R}^{3},
$$

admits a $C^{1}$-family of ground states $\phi_{\omega}(x)$ for $\omega \in \mathcal{O}$.

(H5)

$$
\frac{d}{d \omega}\left\|\phi_{\omega}\right\|_{L^{2}\left(\mathbb{R}^{3}\right)}^{2}>0 \quad \text { for } \omega \in \mathcal{O} .
$$

(H6) Let $L_{+}=-\Delta+V+\omega-\beta\left(\phi_{\omega}^{2}\right)-2 \beta^{\prime}\left(\phi_{\omega}^{2}\right) \phi_{\omega}^{2}$ be the operator whose domain is $H^{2}\left(\mathbb{R}^{3}\right)$. Then $L_{+}$has exactly one negative eigenvalue and does not have kernel.

(H7) Let $\mathcal{H}_{\omega}$ be the linearized operator around $e^{i t \omega} \phi_{\omega}$ (see Section 3 for the precise definition). There is a fixed $m \geq 0$ such that $\mathcal{H}_{\omega}$ has $m$ positive eigenvalues $\lambda_{1}(\omega) \leq \lambda_{2}(\omega) \leq \ldots \leq \lambda_{m}(\omega)$. We assume there are fixed integers $m_{0}=0<m_{1}<\ldots<m_{l_{0}}=m$ such that $\lambda_{j}(\omega)=\lambda_{i}(\omega)$ exactly for $i$ and $j$ both in $\left(m_{l}, m_{l+1}\right]$ for some $l \leq l_{0}$. In this case $\operatorname{dim} \operatorname{ker}\left(\mathcal{H}_{\omega}-\right.$ $\left.\lambda_{j}(\omega)\right)=m_{l+1}-m_{l}$. We assume there exist $N_{j} \in \mathbb{N}$ such that $0<$ $N_{j} \lambda_{j}(\omega)<\omega<\left(N_{j}+1\right) \lambda_{j}(\omega)$ with $N_{j} \geq 1$. We set $N=N_{1}$.

(H8) There is no multi index $\mu \in \mathbb{Z}^{m}$ with $|\mu|:=\left|\mu_{1}\right|+\ldots+\left|\mu_{m}\right| \leq 2 N_{1}+3$ such that $\mu \cdot \lambda=\omega$.

(H9) If $\lambda_{j_{1}}<\ldots<\lambda_{j_{k}}$ are $k$ distinct $\lambda$ 's, and $\mu \in \mathbb{Z}^{k}$ satisfies $|\mu| \leq 2 N_{1}+3$, then we have

$$
\mu_{1} \lambda_{j_{1}}+\cdots+\mu_{k} \lambda_{j_{k}}=0 \Longleftrightarrow \mu=0 .
$$

(H10) $\mathcal{H}_{\omega}$ has no other eigenvalues except for 0 and the $\pm \lambda_{j}(\omega)$. The points $\pm \omega$ are not resonances.

(H11) The Fermi golden rule Hypothesis (H11) in subsection 10.1, see (10.24), holds.

Remark 2.1. The novelty of this paper with respect to $[\mathrm{CM}]$ is that we prove that some crucial coefficients are of a specific form, see (10.24). As a consequence, see Lemma 10.5, these coefficients are positive semidefinite. In the analogue of $(10.24)$ in $[\mathrm{CM}]$, see Hypothesis 5.2 p.72 [CM], except for the special case $n=1$ of just one eigenvalue (or of possibly many eigenvalues but all with $N_{j}=1$ ), there is no clue on the sign of the term on the rhs of the key inequality, and the fact that it is positive is an hypothesis. 
Theorem 2.2. Let $\omega_{1} \in \mathcal{O}$ and $\phi_{\omega_{1}}(x)$ be a ground state of (1.1). Let $u(t, x)$ be a solution to (1.1). Assume (H1)-(H10). Then, there exist an $\epsilon_{0}>0$ and $a C>0$ such that if $\epsilon:=\inf _{\gamma \in[0,2 \pi]}\left\|u_{0}-e^{\mathrm{i} \gamma} \phi_{\omega_{1}}\right\|_{H^{1}}<\epsilon_{0}$, there exist $\omega_{ \pm} \in \mathcal{O}$, $\theta \in C^{1}(\mathbb{R} ; \mathbb{R})$ and $h_{ \pm} \in H^{1}$ with $\left\|h_{ \pm}\right\|_{H^{1}}+\left|\omega_{ \pm}-\omega_{1}\right| \leq C \epsilon$ such that

$$
\lim _{t \rightarrow \pm \infty}\left\|u(t, \cdot)-e^{\mathrm{i} \theta(t)} \phi_{\omega_{ \pm}}-e^{\mathrm{i} t \Delta} h_{ \pm}\right\|_{H^{1}}=0 .
$$

It is possible to write $u(t, x)=e^{\mathrm{i} \theta(t)} \phi_{\omega(t)}+A(t, x)+\widetilde{u}(t, x)$ with $|A(t, x)| \leq$ $C_{N}(t)\langle x\rangle^{-N}$ for any $N$, with $\lim _{|t| \rightarrow \infty} C_{N}(t)=0$, with $\lim _{t \rightarrow \pm \infty} \omega(t)=\omega_{ \pm}$, and such that for any pair $(r, p)$ which is admissible, by which we mean that

$$
2 / r+3 / p=3 / 2, \quad 6 \geq p \geq 2, \quad r \geq 2,
$$

we have

$$
\|\widetilde{u}\|_{L_{t}^{r}\left(\mathbb{R}, W_{x}^{1, p}\right)} \leq C \epsilon .
$$

We end the introduction with some notation. Given two functions $f, g$ : $\mathbb{R}^{3} \rightarrow \mathbb{C}$ we set $\langle f, g\rangle=\int_{\mathbb{R}^{3}} f(x) g(x) d x$. Given a matrix $A$, we denote by $A^{*}$, or by ${ }^{t} A$, its transpose. Given two vectors $A$ and $B$, we denote by $A^{*} B=\sum_{j} A_{j} B_{j}$ their inner product. Sometimes we omit the summation symbol, and we use the convention on sum over repeated indexes. Given two functions $f, g: \mathbb{R}^{3} \rightarrow \mathbb{C}^{2}$ we set $\langle f, g\rangle=\int_{\mathbb{R}^{3}} f^{*}(x) g(x) d x$. For any $k, s \in \mathbb{R}$ and any Banach space $K$ with field $\mathbb{C}$

$$
H^{k, s}\left(\mathbb{R}^{3}, K\right)=\left\{f: \mathbb{R}^{3} \rightarrow K \text { s.t. }\|f\|_{H^{s, k}}:=\left\|\langle x\rangle^{s}\right\|(-\Delta+1)^{k} f\left\|_{K}\right\|_{L^{2}}<\infty\right\},
$$

$(-\Delta+1)^{k} f(x)=(2 \pi)^{-\frac{3}{2}} \int e^{\mathrm{i} x \cdot \xi}\left(\xi^{2}+1\right)^{k} \widehat{f}(\xi) d \xi, \widehat{f}(\xi)=(2 \pi)^{-\frac{3}{2}} \int e^{-\mathrm{i} x \cdot \xi} f(x) d x$. In particular we set $L^{2, s}=H^{0, s}, L^{2}=L^{2,0}, H^{k}=H^{2,0}$. Sometimes, to emphasize that these spaces refer to spatial variables, we will denote them by $W_{x}^{k, p}$, $L_{x}^{p}, H_{x}^{k}, H_{x}^{k, s}$ and $L_{x}^{2, s}$. For $I$ an interval and $Y_{x}$ any of these spaces, we will consider Banach spaces $L_{t}^{p}\left(I, Y_{x}\right)$ with mixed norm $\|f\|_{L_{t}^{p}\left(I, Y_{x}\right)}:=\|\| f\left\|_{Y_{x}}\right\|_{L_{t}^{p}(I)}$. Given an operator $A$, we will denote by $R_{A}(z)=(A-z)^{-1}$ its resolvent. We set $\mathbb{N}_{0}=\mathbb{N} \cup\{0\}$. We will consider multi indexes $\mu \in \mathbb{N}_{0}^{n}$. For $\mu \in \mathbb{Z}^{n}$ with $\mu=\left(\mu_{1}, \ldots, \mu_{n}\right)$ we set $|\mu|=\sum_{j=1}^{n}\left|\mu_{j}\right|$. For $X$ and $Y$ two Banach space, we will denote by $B(X, Y)$ the Banach space of bounded linear operators from $X$ to $Y$ and by $B^{\ell}(X, Y)=B\left(\prod_{j=1}^{\ell} X, Y\right)$. We denote by $a^{\otimes \ell}$ the element $\otimes_{j=1}^{\ell} a$ of $\otimes_{j=1}^{\ell} X$ for some $a \in X$. Given a differential form $\alpha$, we denote by $d \alpha$ its exterior differential.

Acknowledgments I wish to thank Dario Bambusi for pointing out a gap in the proof of an earlier version of Theorem 9.1.

\section{$3 \quad$ Linearization and set up}

Let $U={ }^{t}(u, \bar{u})$. We introduce now energy $E(u)$ and mass $Q(u)$. We set 


$$
\begin{aligned}
& E(U)=E_{K}(U)+E_{P}(U) \\
& E_{K}(U)=\int_{\mathbb{R}^{3}} \nabla u \cdot \nabla \bar{u} d x+\int_{\mathbb{R}^{3}} V u \bar{u} d x \\
& E_{P}(U)=\int_{\mathbb{R}^{3}} B(u \bar{u}) d x
\end{aligned}
$$

with $B(0)=0$ and $\partial_{\bar{u}} B\left(|u|^{2}\right)=\beta\left(|u|^{2}\right) u$. We will consider the matrices

$$
\sigma_{1}=\left(\begin{array}{ll}
0 & 1 \\
1 & 0
\end{array}\right), \sigma_{2}=\left(\begin{array}{cc}
0 & \mathrm{i} \\
-\mathrm{i} & 0
\end{array}\right), \sigma_{3}=\left(\begin{array}{cc}
1 & 0 \\
0 & -1
\end{array}\right) .
$$

We introduce the mass

$$
Q(U)=\int_{\mathbb{R}^{3}} u \bar{u} d x=\frac{1}{2}\left\langle U, \sigma_{1} U\right\rangle .
$$

Let

$$
\Phi_{\omega}=\left(\begin{array}{c}
\phi_{\omega} \\
\phi_{\omega}
\end{array}\right), q(\omega)=Q\left(\Phi_{\omega}\right), e(\omega)=E\left(\Phi_{\omega}\right), d(\omega)=e(\omega)+\omega q(\omega) .
$$

Often we will denote $\Phi_{\omega}$ simply by $\Phi$. The (1.1) can be written as

$$
\mathrm{i} \dot{U}=\left(\begin{array}{cc}
0 & 1 \\
-1 & 0
\end{array}\right)\left(\begin{array}{c}
\partial_{u} E \\
\partial_{\bar{u}} E
\end{array}\right)=\sigma_{3} \sigma_{1} \nabla E(U)
$$

with $\nabla E(U)$ defined by (3.5). We have for $\vartheta \in \mathbb{R}$

$$
E\left(e^{-\mathrm{i} \sigma_{3} \vartheta} U\right)=E(U) \text { and } \nabla E\left(e^{-\mathrm{i} \sigma_{3} \vartheta} U\right)=e^{\mathrm{i} \sigma_{3} \vartheta} \nabla E(U) .
$$

Write for $\omega \in \mathcal{O}$

$$
U=e^{\mathrm{i} \sigma_{3} \vartheta}\left(\Phi_{\omega}+R\right)
$$

Then

$$
\mathrm{i} \dot{U}=-\sigma_{3} \dot{\vartheta} e^{\mathrm{i} \sigma_{3} \vartheta}\left(\Phi_{\omega}+R\right)+\mathrm{i} \dot{\omega} e^{\mathrm{i} \sigma_{3} \vartheta} \partial_{\omega} \Phi_{\omega}+\mathrm{i} e^{\mathrm{i} \sigma_{3} \vartheta} \dot{R}
$$

and

$$
-\sigma_{3} \dot{\vartheta} e^{\mathrm{i} \sigma_{3} \vartheta}\left(\Phi_{\omega}+R\right)+\mathrm{i} \dot{\omega} e^{\mathrm{i} \sigma_{3} \vartheta} \partial_{\omega} \Phi_{\omega}+\mathrm{i} e^{\mathrm{i} \sigma_{3} \vartheta} \dot{R}=\sigma_{3} \sigma_{1} e^{-\mathrm{i} \sigma_{3} \vartheta} \nabla E\left(\Phi_{\omega}+R\right) .
$$

Equivalently we get

$$
\begin{aligned}
& -\sigma_{3}(\dot{\vartheta}-\omega)\left(\Phi_{\omega}+R\right)+\mathrm{i} \dot{\omega} \partial_{\omega} \Phi_{\omega}+\mathrm{i} \dot{R}= \\
& =\sigma_{3} \sigma_{1}\left(\nabla E\left(\Phi_{\omega}+R\right)+\omega \nabla Q\left(\Phi_{\omega}+R\right)\right) .
\end{aligned}
$$

We have $\frac{d}{d t} \sigma_{3} \sigma_{1}\left(\nabla E\left(\Phi_{\omega}+t R\right)+\omega \nabla Q\left(\Phi_{\omega}+t R\right)\right)_{\left.\right|_{t=0}}=\mathcal{H}_{\omega} R$ with

$$
\mathcal{H}_{\omega}=\sigma_{3}(-\Delta+V+\omega)+\sigma_{3}\left[\beta\left(\phi_{\omega}^{2}\right)+\beta^{\prime}\left(\phi_{\omega}^{2}\right) \phi_{\omega}^{2}\right]-\mathrm{i} \sigma_{2} \beta^{\prime}\left(\phi_{\omega}^{2}\right) \phi_{\omega}^{2} .
$$


The essential spectrum of $\mathcal{H}_{\omega}$ consists of $(-\infty,-\omega] \cup[\omega,+\infty)$. It is well known (see [W2]) that by (H5) 0 is an isolated eigenvalue of $\mathcal{H}_{\omega}$ with $\operatorname{dim} N_{g}\left(\mathcal{H}_{\omega}\right)=2$ and

$$
\mathcal{H}_{\omega} \sigma_{3} \Phi_{\omega}=0, \quad \mathcal{H}_{\omega} \partial_{\omega} \Phi_{\omega}=-\Phi_{\omega}
$$

Since $\mathcal{H}_{\omega}^{*}=\sigma_{3} \mathcal{H}_{\omega} \sigma_{3}$, we have $N_{g}\left(\mathcal{H}_{\omega}^{*}\right)=\operatorname{span}\left\{\Phi_{\omega}, \sigma_{3} \partial_{\omega} \Phi_{\omega}\right\}$. We consider eigenfunctions $\xi_{j}(\omega)$ with eigenvalue $\lambda_{j}(\omega)$ :

$$
\mathcal{H}_{\omega} \xi_{j}(\omega)=\lambda_{j}(\omega) \xi_{j}(\omega), \quad \mathcal{H}_{\omega} \sigma_{1} \xi_{j}(\omega)=-\lambda_{j}(\omega) \sigma_{1} \xi_{j}(\omega) .
$$

They can be normalized so that $\left\langle\sigma_{3} \xi_{j}(\omega), \bar{\xi}_{\ell}(\omega)\right\rangle=\delta_{j \ell}$, this is based on Proposition $2.4[\mathrm{Cu} 3]$. Furthermore, they can be chosen to be real, that is with real entries, so $\xi_{j}=\bar{\xi}_{j}$ for all $j$.

Both $\phi_{\omega}$ and $\xi_{j}(\omega, x)$ are smooth in $\omega \in \mathcal{O}$ and $x \in \mathbb{R}^{3}$ and satisfy

$$
\sup _{\omega \in \mathcal{K}, x \in \mathbb{R}^{3}} e^{a|x|}\left(\left|\partial_{x}^{\alpha} \phi_{\omega}(x)\right|+\sum_{j=1}^{m}\left|\partial_{x}^{\alpha} \xi_{j}(\omega, x)\right|<\infty\right.
$$

for every $a \in\left(0, \inf _{\omega \in \mathcal{K}} \sqrt{\omega-\lambda_{m}(\omega)}\right)$ and every compact subset $\mathcal{K}$ of $\mathcal{O}$.

For $\omega \in \mathcal{O}$, we have the $\mathcal{H}_{\omega}$-invariant Jordan block decomposition

$$
L^{2}\left(\mathbb{R}^{3}, \mathbb{C}^{2}\right)=N_{g}\left(\mathcal{H}_{\omega}\right) \oplus\left(\oplus_{ \pm} \oplus_{j=1}^{m} \operatorname{ker}\left(\mathcal{H}_{\omega} \mp \lambda_{j}(\omega)\right)\right) \oplus L_{c}^{2}\left(\mathcal{H}_{\omega}\right),
$$

$L_{c}^{2}\left(\mathcal{H}_{\omega}\right):=\left\{N_{g}\left(\mathcal{H}_{\omega}^{*}\right) \oplus\left(\oplus_{\lambda \in \sigma_{d} \backslash\{0\}} \operatorname{ker}\left(\mathcal{H}_{\omega}^{*}-\lambda\right)\right)\right\}^{\perp}$ with $\sigma_{d}=\sigma_{d}\left(\mathcal{H}_{\omega}\right)$. We also set $L_{d}^{2}\left(\mathcal{H}_{\omega}\right):=N_{g}\left(\mathcal{H}_{\omega}\right) \oplus\left(\oplus_{\lambda \in \sigma_{d} \backslash\{0\}} \operatorname{ker}\left(\mathcal{H}_{\omega}-\lambda(\omega)\right)\right)$. By $P_{c}\left(\mathcal{H}_{\omega}\right)$ (resp. $\left.P_{d}\left(\mathcal{H}_{\omega}\right)\right)$, or simply by $P_{c}(\omega)$ (resp. $\left.P_{d}(\omega)\right)$, we denote the projection on $L_{c}^{2}\left(\mathcal{H}_{\omega}\right)$ (resp. $\left.L_{d}^{2}\left(\mathcal{H}_{\omega}\right)\right)$ associated to the above direct sum. The space $L_{c}^{2}\left(\mathcal{H}_{\omega}\right)$ depends continuously on $\omega$. We specify the ansatz imposing that

$$
U=e^{\mathrm{i} \sigma_{3} \vartheta}\left(\Phi_{\omega}+R\right) \text { with } \omega \in \mathcal{O}, \vartheta \in \mathbb{R} \text { and } R \in N_{g}^{\perp}\left(\mathcal{H}_{\omega}^{*}\right) .
$$

We consider coordinates

$$
U=e^{\mathrm{i} \sigma_{3} \vartheta}\left(\Phi_{\omega}+z \cdot \xi(\omega)+\bar{z} \cdot \sigma_{1} \xi(\omega)+P_{c}\left(\mathcal{H}_{\omega}\right) f\right)
$$

where $\omega \in \mathcal{O}, z \in \mathbb{C}$ and $f \in L_{c}^{2}\left(\mathcal{H}_{\omega_{0}}\right)$ where we fixed $\omega_{0} \in \mathcal{O}$ such that $q\left(\omega_{0}\right)=\left\|u_{0}\right\|_{2}^{2}$. (3.13) is a system of coordinates if we use the notation $\mathcal{O}$ to denote a small neighborhood of $\omega_{1}$ in Theorem 2.2. Indeed by Lemma 3.1 below, then the map $P_{c}\left(\mathcal{H}_{\omega}\right)$ is an isomorphism from $L_{c}^{2}\left(\mathcal{H}_{\omega_{0}}\right)$ to $L_{c}^{2}\left(\mathcal{H}_{\omega}\right)$. In particular

$$
\begin{aligned}
& R=\sum_{j=1}^{m} z_{j} \xi_{j}(\omega)+\sum_{j=1}^{m} \bar{z}_{j} \sigma_{1} \xi_{j}(\omega)+P_{c}\left(\mathcal{H}_{\omega}\right) f, \\
& R \in N_{g}^{\perp}\left(\mathcal{H}_{\omega}^{*}\right) \text { and } f \in L_{c}^{2}\left(\mathcal{H}_{\omega_{0}}\right) .
\end{aligned}
$$

We also set $z \cdot \xi=\sum_{j} z_{j} \xi_{j}$ and $\bar{z} \cdot \sigma_{1} \xi=\sum_{j} \bar{z}_{j} \sigma_{1} \xi_{j}$. In the sequel we set

$$
\partial_{\omega} R=\sum_{j=1}^{m} z_{j} \partial_{\omega} \xi_{j}(\omega)+\sum_{j=1}^{m} \bar{z}_{j} \sigma_{1} \partial_{\omega} \xi_{j}(\omega)+\partial_{\omega} P_{c}\left(\mathcal{H}_{\omega}\right) f .
$$

We have: 
Lemma 3.1. We have $P_{c}\left(\mathcal{H}_{\omega}\right)^{*}=P_{c}\left(\mathcal{H}_{\omega}^{*}\right)$ for all $\omega \in \mathcal{O}$. For all $\omega, \widetilde{\omega} \in \mathcal{O}$ the following operators are bounded from $H^{-k,-s}$ to $H^{k^{\prime}, s^{\prime}}$ for all exponents:

$$
\begin{aligned}
& \partial_{\omega}^{\ell} P_{c}\left(\mathcal{H}_{\omega}\right) \text { for any } \ell>0 ; \\
& P_{c}\left(\mathcal{H}_{\omega}\right)-P_{c}\left(\mathcal{H}_{\omega}^{*}\right) ; P_{c}\left(\mathcal{H}_{\omega}\right)-P_{c}\left(\mathcal{H}_{\widetilde{\omega}}\right) .
\end{aligned}
$$

Consider $\omega_{1}$ of Theorem 2.2. There exists $\varepsilon_{1}>0$ such that $\left(\omega_{1}-\varepsilon_{1}, \omega_{1}+\varepsilon_{1}\right) \subset \mathcal{O}$, and for any pair $\widetilde{\omega}, \omega \in\left(\omega_{1}-\varepsilon_{1}, \omega_{1}+\varepsilon_{1}\right)$ we have

$$
P_{c}(\omega) P_{c}(\widetilde{\omega}): L_{c}^{2}\left(\mathcal{H}_{\widetilde{\omega}}\right) \rightarrow L_{c}^{2}\left(\mathcal{H}_{\omega}\right) \text { is an isomorphism }
$$

Furthermore, the following operator is bounded from $H^{-k,-s}$ to $H^{k^{\prime}, s^{\prime}}$ for all exponents:

$$
P_{c}\left(\mathcal{H}_{\widetilde{\omega}}\right)\left(1-\left(P_{c}\left(\mathcal{H}_{\omega}\right) P_{c}\left(\mathcal{H}_{\widetilde{\omega}}\right)\right)^{-1}\right) P_{c}\left(\mathcal{H}_{\omega}\right)
$$

where in the last line and $\left(P_{c}(\omega) P_{c}(\widetilde{\omega})\right)^{-1}$ is the inverse of the operator in (3.18). Finally, for $\epsilon_{0}$ in Theorem 2.2 sufficiently small, we have $\left|\omega_{0}-\omega_{1}\right|<\varepsilon_{1}$, with $\omega_{0}$ defined under (3.13).

Proof. The first statement follows from the definition. We have $P_{c}\left(\mathcal{H}_{\omega}\right)=$ $1-P_{d}\left(\mathcal{H}_{\omega}\right)$ where $P_{d}\left(\mathcal{H}_{\omega}\right)$ are finite linear combinations of rank 1 operators $\Psi\left\langle\Psi^{\prime}, \quad\right\rangle$ with $\Psi, \Psi^{\prime} \in H^{K, S}$ for any $(K, S)$. This implies the statement for the second line of (3.17). $\partial_{\omega}^{\ell} P_{c}\left(\mathcal{H}_{\omega}\right)$ is well defined by the fact that in (H4) the dependence on $\omega$ is in fact smooth (this is seen iterating the argument in Theorem $18[\mathrm{ShS}])$. Assuming (3.18), and for $P_{c}=P_{c}(\omega), \widetilde{P}_{c}=P_{c}(\widetilde{\omega})$, $P_{d}=P_{d}(\omega), \widetilde{P}_{d}=P_{d}(\widetilde{\omega})$, we have

$$
\widetilde{P}_{c}\left(1-\left(P_{c} \widetilde{P}_{c}\right)^{-1}\right) P_{c}=\left(\widetilde{P}_{c}-P_{c}\right) P_{c}-\left(\widetilde{P}_{c}-P_{c}\right)\left(P_{c} \widetilde{P}_{c}\right)^{-1} P_{c},
$$

which yields (3.19). We prove (3.18). First of all the map is 1-1. Indeed if $P_{c} \widetilde{P}_{c} f=0$, then we have $f=P_{d} f=\left(P_{d}-\widetilde{P}_{d}\right) f$. Then $\|f\|_{2} \leq C|\omega-\widetilde{\omega}|\|f\|_{2}$ for some fixed $C>0$. This, for $2 C \varepsilon_{1}<1$, is compatible only with $f=0$. If we knew that the map in (3.18) is onto, then (3.18) would hold by the open mapping theorem. So suppose the map is not onto. Let $\mathcal{R}\left(P_{c} \widetilde{P}_{c}\right)$ be the range of $P_{c} \widetilde{P}_{c}$. If there exists $\widetilde{g} \in L_{c}^{2}\left(\mathcal{H}_{\omega}^{*}\right)$ such that $\widetilde{g} \neq 0$ and $\left\langle\widetilde{g}, P_{c} \widetilde{P}_{c} f\right\rangle=0$ for all $f \in L_{c}^{2}\left(\mathcal{H}_{\widetilde{\omega}}\right)$, then since $\widetilde{g}=\sigma_{3} g$ for a $g \in L_{c}^{2}\left(\mathcal{H}_{\omega}\right)$, we get $0=\left\langle\widetilde{g}, P_{c} \widetilde{P}_{c} f\right\rangle=\left\langle\widetilde{P}_{c} P_{c} g, \sigma_{3} f\right\rangle$ for all $f \in L_{c}^{2}\left(\mathcal{H}_{\widetilde{\omega}}\right)$. This implies $\widetilde{P}_{c} P_{c} g=0$, and since $g \in L_{c}^{2}\left(\mathcal{H}_{\omega}\right)$, by the $1-1$ argument this implies $g=0$. So if the map in (3.18) is not onto, then $\mathcal{R}\left(P_{c} \widetilde{P}_{c}\right)$ is dense in $L_{c}^{2}\left(\mathcal{H}_{\omega}\right)$. We will see in a moment that $\mathcal{R}\left(P_{c} \widetilde{P}_{c}\right)$ is closed in $L_{c}^{2}\left(\mathcal{H}_{\omega}\right)$, hence concluding that the map in (3.18) is also onto. To see that $\mathcal{R}\left(P_{c} \widetilde{P}_{c}\right)$ is closed in $L_{c}^{2}\left(\mathcal{H}_{\omega}\right)$, let $\widetilde{f}_{n} \in L_{c}^{2}\left(\mathcal{H}_{\widetilde{\omega}}\right)$ be a sequence such that $\left\|P_{c} \widetilde{f}_{n}-f\right\|_{2} \rightarrow 0$ for some $f \in L_{c}^{2}\left(\mathcal{H}_{\omega}\right)$. By $\left\|\widetilde{f}_{n}\right\|_{2} \leq\left\|P_{c} \widetilde{f}_{n}\right\|_{2}+C \mid \omega-\widetilde{\omega}\left\|\widetilde{f}_{n}\right\|_{2}$ for some fixed $C$, it follows that for $2 C \varepsilon_{1}<1$ the sequence $\left\|\widetilde{f}_{n}\right\|_{2}$ is bounded. Then by weak compactness there is a subsequence $\widetilde{f}_{n_{j}}$ weakly convergent to a $\widetilde{f} \in L_{c}^{2}\left(\mathcal{H}_{\widetilde{\omega}}\right)$. Since $P_{c} \widetilde{P}_{c}$ is also weakly continuous, $P_{c} \widetilde{P}_{c} \widetilde{f}=f$.

The final statement is elementary by $(2.2)$. 
Using the system of coordinates (3.13) we rewrite system (3.8) as

$$
\begin{aligned}
& -\sigma_{3}(\dot{\vartheta}-\omega)\left(\Phi_{\omega}+z \cdot \xi+\bar{z} \cdot \sigma_{1} \xi+P_{c}\left(\mathcal{H}_{\omega}\right) f\right)+ \\
& +\mathrm{i} \dot{\omega}\left(\partial_{\omega} \Phi_{\omega}+z \cdot \partial_{\omega} \xi+\bar{z} \cdot \sigma_{1} \partial_{\omega} \xi+\partial_{\omega} P_{c}\left(\mathcal{H}_{\omega}\right) f\right)+\mathrm{i} \dot{z} \cdot \xi+ \\
& +\mathrm{i} \dot{\bar{z}} \cdot \sigma_{1} \xi+\mathrm{i} P_{c}\left(\mathcal{H}_{\omega}\right) \dot{f}=\sigma_{3} \sigma_{1} \nabla E\left(\Phi_{\omega}+z \cdot \xi+\bar{z} \cdot \sigma_{1} \xi+P_{c}\left(\mathcal{H}_{\omega}\right) f\right) \\
& +\omega \sigma_{3} \sigma_{1} \nabla Q\left(\Phi_{\omega}+z \cdot \xi+\bar{z} \cdot \sigma_{1} \xi+P_{c}\left(\mathcal{H}_{\omega}\right) f\right),
\end{aligned}
$$

where $z \cdot \xi=\sum_{j} z_{j} \xi_{j}$ and $\bar{z} \cdot \sigma_{1} \xi=\sum_{j} \bar{z}_{j} \sigma_{1} \xi_{j}$, where $\xi=\xi(\omega)$. Notice for future reference, that fixed any $\omega_{0}$ we also have

$$
\begin{aligned}
& -\sigma_{3}\left(\dot{\vartheta}-\omega_{0}\right)\left(\Phi_{\omega}+z \cdot \xi+\bar{z} \cdot \sigma_{1} \xi+P_{c}\left(\mathcal{H}_{\omega}\right) f\right)+ \\
& +\mathrm{i} \dot{\omega}\left(\partial_{\omega} \Phi_{\omega}+z \cdot \partial_{\omega} \xi+\bar{z} \cdot \sigma_{1} \partial_{\omega} \xi+\partial_{\omega} P_{c}\left(\mathcal{H}_{\omega}\right) f\right)+\mathrm{i} \dot{z} \cdot \xi+ \\
& +\mathrm{i} \dot{\bar{z}} \cdot \sigma_{1} \xi+\mathrm{i} P_{c}\left(\mathcal{H}_{\omega}\right) \dot{f}=\sigma_{3} \sigma_{1} \nabla E\left(\Phi_{\omega}+z \cdot \xi+\bar{z} \cdot \sigma_{1} \xi+P_{c}\left(\mathcal{H}_{\omega}\right) f\right) \\
& +\omega_{0} \sigma_{3} \sigma_{1} \nabla Q\left(\Phi_{\omega}+z \cdot \xi+\bar{z} \cdot \sigma_{1} \xi+P_{c}\left(\mathcal{H}_{\omega}\right) f\right),
\end{aligned}
$$

where (3.21) is the same of (3.20) except for $\omega_{0}$ replacing $\omega$ in the first spot where they appear in the first and last line.

We end this section with a short heuristic description about why the crucial property needed to prove asymptotic stability of ground states, is the hamiltonian nature of the (1.1). In terms of (3.13), and oversimplifying, (3.7) splits as

$$
\begin{aligned}
& \mathrm{i} \dot{z}-\lambda z=\sum_{\mu \nu} a_{\mu \nu} z^{\mu} \bar{z}^{\nu}+\sum_{\mu \nu} z^{\mu} \bar{z}^{\nu}\left\langle f(t, x), \bar{G}_{\mu \nu}(x, \omega)\right\rangle_{L_{x}^{2}}+\cdots \\
& \mathrm{i} \dot{f}-\mathcal{H}_{\omega} f=\sum_{\mu \nu} z^{\mu} \bar{z}^{\nu} M_{\mu \nu}(x, \omega)+\cdots
\end{aligned}
$$

Here we are assuming $m=1$. We focus on positive times $t \geq 0$ only. After changes of variables, see $[\mathrm{CM}]$, we obtain

$$
\begin{aligned}
& \mathrm{i} \dot{z}-\lambda z=P\left(|z|^{2}\right) z+\bar{z}^{N}\left\langle f(t, x), \bar{G}_{\mu \nu}(x, \omega)\right\rangle_{L_{x}^{2}}+\cdots \\
& \mathrm{i} \dot{f}-\mathcal{H}_{\omega} f=z^{N+1} M(x, \omega)+\cdots .
\end{aligned}
$$

The next step is to write, for $g$ an error term,

$$
\begin{aligned}
& f=-z^{N+1} R_{\mathcal{H}_{\omega}}^{+}((N+1) \lambda) M+g \\
& \mathrm{i} \dot{z}-\lambda z=P\left(|z|^{2}\right) z-|z|^{2 N} z\left\langle R_{\mathcal{H}_{\omega}}^{+}((N+1) \lambda) M, \bar{G}\right\rangle_{L_{x}^{2}}+\ldots
\end{aligned}
$$

Then, ignoring error terms, by

$$
R_{\mathcal{H}_{\omega}}^{+}((N+1) \lambda)=P \cdot V \cdot \frac{1}{\mathcal{H}_{\omega}-(N+1) \lambda}+\mathrm{i} \pi \delta\left(\mathcal{H}_{\omega}-(N+1) \lambda\right)
$$

the equation for $z$ has solutions such that

$$
\frac{d}{d t}|z|^{2}=-\Gamma|z|^{2 N+2},|z(t)|=\frac{|z(0)|}{\left(|z(0)|^{2 N} N \Gamma t+1\right)^{\frac{1}{2 N}}}
$$


with (the Fourier transforms are associated to $\mathcal{H}_{\omega}$ )

$$
\Gamma=2 \pi\left\langle\delta\left(\mathcal{H}_{\omega}-(N+1) \lambda\right) M, G\right\rangle=\int_{|\xi|=\sqrt{(N+1) \lambda-\omega}} \frac{\widehat{M}(\xi) \cdot \overline{\widehat{G}(\xi)} d \sigma}{\sqrt{(N+1) \lambda-\omega}} .
$$

If $\Gamma>0$, we see that $z(t)$ decays. Notice that $\Gamma<0$ is incompatible with orbital stability, which requires $z$ to remain small, see Corollary $4.6[\mathrm{CM}]$. The latter indirect argument to prove positive semidefiniteness of $\Gamma$, does not seem to work when in (3.7) there are further discrete components. So we need another way to prove that $\Gamma \geq 0$. This is provided by the hamiltonian structure. Indeed, if (3.22) is of the form

$$
\mathrm{i} \dot{z}=\partial_{\bar{z}} K, \quad \mathrm{i} \dot{f}=\nabla_{\bar{f}} K
$$

then by Schwartz lemma $(N+1) ! M=\partial_{z}^{N+1} \nabla_{\bar{f}} K=\overline{\partial_{\bar{z}}^{N} \nabla_{f} \partial_{\bar{z}} K}=N ! G$ at $z=0$ and $f=0$. So $\Gamma$ is positive semidefinite. This very simple idea on system (3.23), inspired $[\mathrm{BC}]$ and inspires the present paper.

\section{Gradient of the coordinates}

We focus on ansatz (3.12) and on the coordinates (3.13). In particular we compute the gradient of the coordinates. Here we recall that given a scalar valued function $F$, the relation between exterior differential and gradient is $d F=\langle\nabla F, \quad\rangle$. Consider the following two functions

$$
\mathcal{F}(U, \omega, \vartheta):=\left\langle e^{-\mathrm{i} \sigma_{3} \vartheta} U-\Phi_{\omega}, \Phi_{\omega}\right\rangle \text { and } \mathcal{G}(U, \omega, \vartheta):=\left\langle e^{-\mathrm{i} \sigma_{3} \vartheta} U, \sigma_{3} \partial_{\omega} \Phi_{\omega}\right\rangle .
$$

Then ansatz $(3.12)$ is obtained by choosing $(\omega, \vartheta)$ s.t. $R:=e^{-\mathrm{i} \sigma_{3} \vartheta} U-\Phi_{\omega}$ satisfies $R \in N_{g}^{\perp}\left(\mathcal{H}_{\omega}^{*}\right)$ by means of the implicit function theorem. In particular:

$$
\begin{aligned}
& \mathcal{F}_{\vartheta}=-\mathrm{i}\left\langle\sigma_{3} e^{-\mathrm{i} \sigma_{3} \vartheta} U, \Phi_{\omega}\right\rangle=-\mathrm{i}\left\langle\sigma_{3} R, \Phi_{\omega}\right\rangle ; \\
& \mathcal{F}_{\omega}=-2 q^{\prime}(\omega)+\left\langle e^{-\mathrm{i} \sigma_{3} \vartheta} U, \partial_{\omega} \Phi_{\omega}\right\rangle=-q^{\prime}(\omega)+\left\langle R, \partial_{\omega} \Phi_{\omega}\right\rangle ; \\
& \nabla_{U} \mathcal{F}=e^{-\mathrm{i} \sigma_{3} \vartheta} \Phi_{\omega}, \nabla_{U} \mathcal{G}=e^{-\mathrm{i} \sigma_{3} \vartheta} \sigma_{3} \partial_{\omega} \Phi_{\omega} ; \\
& \mathcal{G}_{\vartheta}=-\mathrm{i}\left\langle e^{-\mathrm{i} \sigma_{3} \vartheta} U, \partial_{\omega} \Phi_{\omega}\right\rangle=-\mathrm{i}\left(q^{\prime}(\omega)+\left\langle R, \partial_{\omega} \Phi_{\omega}\right\rangle\right) ; \\
& \mathcal{G}_{\omega}=\left\langle e^{-\mathrm{i} \sigma_{3} \vartheta} U, \sigma_{3} \partial_{\omega}^{2} \Phi_{\omega}\right\rangle=\left\langle R, \sigma_{3} \partial_{\omega}^{2} \Phi_{\omega}\right\rangle .
\end{aligned}
$$

By $\mathcal{F}\left(U, \omega(U), \vartheta(U)=\mathcal{G}\left(U, \omega(U), \vartheta(U)=0\right.\right.$ we get $\mathcal{W}_{\omega} \nabla \omega+\mathcal{W}_{\vartheta} \nabla \vartheta=-\nabla_{U} W$ for $\mathcal{W}=\mathcal{F}, \mathcal{G}$. By the above formulas, if we set

$$
\mathcal{A}=\left(\begin{array}{cc}
-q^{\prime}(\omega)+\left\langle R, \partial_{\omega} \Phi_{\omega}\right\rangle & -\mathrm{i}\left\langle\sigma_{3} R, \Phi_{\omega}\right\rangle \\
\left\langle R, \sigma_{3} \partial_{\omega}^{2} \Phi_{\omega}\right\rangle & -\mathrm{i}\left(q^{\prime}(\omega)+\left\langle R, \partial_{\omega} \Phi_{\omega}\right\rangle\right)
\end{array}\right)
$$

we have

$$
\mathcal{A}\left(\begin{array}{c}
\nabla \omega \\
\nabla \vartheta
\end{array}\right)=\left(\begin{array}{c}
-e^{-\mathrm{i} \sigma_{3} \vartheta} \Phi_{\omega} \\
-e^{-\mathrm{i} \sigma_{3} \vartheta} \sigma_{3} \partial_{\omega} \Phi_{\omega}
\end{array}\right)
$$


So

$$
\begin{aligned}
& \nabla \omega=\frac{\left(q^{\prime}(\omega)+\left\langle R, \partial_{\omega} \Phi_{\omega}\right\rangle\right) e^{-\mathrm{i} \sigma_{3} \vartheta} \Phi_{\omega}-\left\langle\sigma_{3} R, \Phi_{\omega}\right\rangle e^{-\mathrm{i} \sigma_{3} \vartheta} \sigma_{3} \partial_{\omega} \Phi_{\omega}}{\left(q^{\prime}(\omega)\right)^{2}-\left\langle R, \partial_{\omega} \Phi_{\omega}\right\rangle^{2}+\left\langle\sigma_{3} R, \Phi_{\omega}\right\rangle\left\langle R, \sigma_{3} \partial_{\omega}^{2} \Phi_{\omega}\right\rangle} \\
& \nabla \vartheta=\frac{\left\langle R, \sigma_{3} \partial_{\omega}^{2} \Phi_{\omega}\right\rangle e^{-\mathrm{i} \sigma_{3} \vartheta} \Phi_{\omega}+\left(q^{\prime}(\omega)-\left\langle R, \partial_{\omega} \Phi_{\omega}\right\rangle\right) e^{-\mathrm{i} \sigma_{3} \vartheta} \sigma_{3} \partial_{\omega} \Phi_{\omega}}{\left.\mathrm{i}\left[q^{\prime}(\omega)\right)^{2}-\left\langle R, \partial_{\omega} \Phi_{\omega}\right\rangle^{2}+\left\langle\sigma_{3} R, \Phi_{\omega}\right\rangle\left\langle R, \sigma_{3} \partial_{\omega}^{2} \Phi_{\omega}\right\rangle\right]} .
\end{aligned}
$$

Notice that along with the decomposition (3.11) we have

$$
L^{2}\left(\mathbb{R}^{3}, \mathbb{C}^{2}\right)=N_{g}\left(\mathcal{H}_{\omega}^{*}\right) \oplus\left(\oplus_{\lambda \in \sigma_{d} \backslash\{0\}} \operatorname{ker}\left(\mathcal{H}_{\omega}^{*}-\lambda(\omega)\right)\right) \oplus L_{c}^{2}\left(\mathcal{H}_{\omega}^{*}\right),
$$

$L_{c}^{2}\left(\mathcal{H}_{\omega}^{*}\right):=\left\{N_{g}\left(\mathcal{H}_{\omega}\right) \oplus\left(\oplus_{\lambda \in \sigma_{d} \backslash\{0\}} \operatorname{ker}\left(\mathcal{H}_{\omega}-\lambda(\omega)\right)\right)\right\}^{\perp}$. We also set $L_{d}^{2}\left(\mathcal{H}_{\omega}^{*}\right):=$ $N_{g}\left(\mathcal{H}_{\omega}^{*}\right) \oplus\left(\oplus_{\lambda \in \sigma_{d} \backslash\{0\}} \operatorname{ker}\left(\mathcal{H}_{\omega}^{*}-\lambda(\omega)\right)\right)$. Notice that $N_{g}\left(\mathcal{H}_{\omega}^{*}\right)=\sigma_{3} N_{g}\left(\mathcal{H}_{\omega}\right)$, $\operatorname{ker}\left(\mathcal{H}_{\omega}^{*}-\lambda\right)=\sigma_{3} \operatorname{ker}\left(\mathcal{H}_{\omega}-\lambda\right), L_{c}^{2}\left(\mathcal{H}_{\omega}^{*}\right)=\sigma_{3} L_{c}^{2}\left(\mathcal{H}_{\omega}\right)$ and $L_{d}^{2}\left(\mathcal{H}_{\omega}^{*}\right)=\sigma_{3} L_{d}^{2}\left(\mathcal{H}_{\omega}\right)$, so that (4.4) is obtained applying $\sigma_{3}$ to decomposition (3.11). We can decompose gradients as

$$
\begin{aligned}
& \nabla F(U)=e^{-\mathrm{i} \sigma_{3} \vartheta}\left[P_{N_{g}\left(\mathcal{H}_{\omega}^{*}\right)}+\right. \\
& \left.\sum_{j}\left(P_{\operatorname{ker}\left(\mathcal{H}_{\omega}^{*}-\lambda_{j}\right)}+P_{\operatorname{ker}\left(\mathcal{H}_{\omega}^{*}+\lambda_{j}\right)}\right)+P_{c}\left(\mathcal{H}_{\omega}^{*}\right)\right] e^{\mathrm{i} \sigma_{3} \vartheta} \nabla F(U)= \\
& \frac{\left\langle\nabla F(U), e^{\mathrm{i} \sigma_{3} \vartheta} \partial_{\omega} \Phi\right\rangle}{q^{\prime}(\omega)} e^{-\mathrm{i} \sigma_{3} \vartheta} \Phi+\frac{\left\langle\nabla F(U), e^{\mathrm{i} \sigma_{3} \vartheta} \sigma_{3} \Phi\right\rangle}{q^{\prime}(\omega)} e^{-\mathrm{i} \sigma_{3} \vartheta} \sigma_{3} \partial_{\omega} \Phi \\
& +\sum_{j}\left\langle\nabla F(U), e^{\mathrm{i} \sigma_{3} \vartheta} \xi_{j}\right\rangle e^{-\mathrm{i} \sigma_{3} \vartheta} \sigma_{3} \xi_{j}+\sum_{j}\left\langle\nabla F(U), e^{\mathrm{i} \sigma_{3} \vartheta} \sigma_{1} \xi_{j}\right\rangle e^{-\mathrm{i} \sigma_{3} \vartheta} \sigma_{1} \sigma_{3} \xi_{j} \\
& +e^{-\mathrm{i} \sigma_{3} \vartheta} P_{c}\left(\mathcal{H}_{\omega}^{*}\right) e^{\mathrm{i} \sigma_{3} \vartheta} \nabla F(U) .
\end{aligned}
$$

Using coordinates (3.13) and notation(3.16), at $U$ we have the following formulas for the vectorfields

$$
\begin{aligned}
& \frac{\partial}{\partial \omega}=e^{\mathrm{i} \sigma_{3} \vartheta} \partial_{\omega}(\Phi+R), \frac{\partial}{\partial \vartheta}=\mathrm{i} e^{\mathrm{i} \sigma_{3} \vartheta} \sigma_{3}(\Phi+R), \\
& \frac{\partial}{\partial z_{j}}=e^{\mathrm{i} \sigma_{3} \vartheta} \xi_{j}, \frac{\partial}{\partial \bar{z}_{j}}=e^{\mathrm{i} \sigma_{3} \vartheta} \sigma_{1} \xi_{j} .
\end{aligned}
$$

Hence, by $\partial_{\omega} F=d F\left(\frac{\partial}{\partial \omega}\right)=\left\langle\nabla F, \frac{\partial}{\partial \omega}\right\rangle$ etc., we have

$$
\begin{aligned}
& \partial_{\omega} F=\left\langle\nabla F, e^{\mathrm{i} \sigma_{3} \vartheta} \partial_{\omega}(\Phi+R)\right\rangle, \partial_{\vartheta} F=\mathrm{i}\left\langle\nabla F, e^{\mathrm{i} \sigma_{3} \vartheta} \sigma_{3}(\Phi+R)\right\rangle, \\
& \partial_{z_{j}} F=\left\langle\nabla F, e^{\mathrm{i} \sigma_{3} \vartheta} \xi_{j}\right\rangle, \partial_{\bar{z}_{j}} F=\left\langle\nabla F, e^{\mathrm{i} \sigma_{3} \vartheta} \sigma_{1} \xi_{j}\right\rangle .
\end{aligned}
$$

Lemma 4.1. We have the following formulas:

$$
\begin{gathered}
\nabla z_{j}=-\left\langle\sigma_{3} \xi_{j}, \partial_{\omega} R\right\rangle \nabla \omega-\mathrm{i}\left\langle\sigma_{3} \xi_{j}, \sigma_{3} R\right\rangle \nabla \vartheta+e^{-\mathrm{i} \sigma_{3} \vartheta} \sigma_{3} \xi_{j} \\
\nabla \bar{z}_{j}=-\left\langle\sigma_{1} \sigma_{3} \xi_{j}, \partial_{\omega} R\right\rangle \nabla \omega-\mathrm{i}\left\langle\sigma_{1} \sigma_{3} \xi_{j}, \sigma_{3} R\right\rangle \nabla \vartheta+e^{-\mathrm{i} \sigma_{3} \vartheta} \sigma_{1} \sigma_{3} \xi_{j} .
\end{gathered}
$$


Proof. Equalities $\frac{\partial z_{j}}{\partial z_{\ell}}=\delta_{j \ell}, \frac{\partial z_{j}}{\partial \bar{z}_{\ell}}=\frac{\partial z_{j}}{\partial \omega}=\frac{\partial z_{j}}{\partial \vartheta}=0$ and $\nabla_{f} z_{j}=0$ are equivalent to

$$
\begin{aligned}
& \left\langle\nabla z_{j}, e^{\mathrm{i} \sigma_{3} \vartheta} \xi_{\ell}\right\rangle=\delta_{j \ell},\left\langle\nabla z_{j}, e^{\mathrm{i} \sigma_{3} \vartheta} \sigma_{1} \xi_{\ell}\right\rangle \equiv 0=\left\langle\nabla z_{j}, e^{\mathrm{i} \sigma_{3} \vartheta} \sigma_{3}(\Phi+R)\right\rangle \\
& \left\langle\nabla z_{j}, e^{\mathrm{i} \sigma_{3} \vartheta} \partial_{\omega}(\Phi+R)\right\rangle=0 \equiv\left\langle\nabla z_{j}, e^{\mathrm{i} \sigma_{3} \vartheta} P_{c}(\omega) P_{c}\left(\omega_{0}\right) g\right\rangle \forall g \in L_{c}^{2}\left(\mathcal{H}_{\omega_{0}}\right) .
\end{aligned}
$$

Notice that the last identity implies $P_{c}\left(\mathcal{H}_{\omega_{0}}^{*}\right) P_{c}\left(\mathcal{H}_{\omega}^{*}\right) e^{\mathrm{i} \sigma_{3} \vartheta} \nabla z_{j}=0$ which in turn implies $P_{c}\left(\mathcal{H}_{\omega}^{*}\right) e^{\mathrm{i} \sigma_{3} \vartheta} \nabla z_{j}=0$. Then, applying (4.5) and using the product row column, we get for some pair of numbers $(a, b)$

$$
\begin{aligned}
& \nabla z_{j}=a e^{-\mathrm{i} \sigma_{3} \vartheta} \Phi+b e^{-\mathrm{i} \sigma_{3} \vartheta} \sigma_{3} \partial_{\omega} \Phi+e^{-\mathrm{i} \sigma_{3} \vartheta} \sigma_{3} \xi_{j} \\
& =(a, b)\left(\begin{array}{c}
e^{-\mathrm{i} \sigma_{3} \vartheta} \Phi \\
e^{-\mathrm{i} \sigma_{3} \vartheta} \sigma_{3} \partial_{\omega} \Phi
\end{array}\right)+e^{-\mathrm{i} \sigma_{3} \vartheta} \sigma_{3} \xi_{j}=-(a, b) \mathcal{A}\left(\begin{array}{c}
\nabla \omega \\
\nabla \vartheta
\end{array}\right)+e^{-\mathrm{i} \sigma_{3} \vartheta} \sigma_{3} \xi_{j},
\end{aligned}
$$

where in the last line we used (4.2). Equating the two extreme sides and applying to the formula $\left\langle, \frac{\partial}{\partial \omega}\right\rangle$ and $\left\langle, \frac{\partial}{\partial \vartheta}\right\rangle$, by $\left\langle\nabla z_{j}, \frac{\partial}{\partial \omega}\right\rangle=\left\langle\nabla z_{j}, \frac{\partial}{\partial \vartheta}\right\rangle=\left\langle\nabla \vartheta, \frac{\partial}{\partial \omega}\right\rangle=$ $\left\langle\nabla \omega, \frac{\partial}{\partial \vartheta}\right\rangle=0$, by $\left\langle\nabla \vartheta, \frac{\partial}{\partial \vartheta}\right\rangle=\left\langle\nabla \omega, \frac{\partial}{\partial \omega}\right\rangle=1$ and by (4.6) and (4.10), we get

$$
\mathcal{A}^{*}\left(\begin{array}{l}
a \\
b
\end{array}\right)=\left(\begin{array}{c}
\left\langle\sigma_{3} \xi_{j}, \partial_{\omega} R\right\rangle \\
\mathrm{i}\left\langle\sigma_{3} \xi_{j}, \sigma_{3} R\right\rangle
\end{array}\right)
$$

This implies

$$
\nabla z_{j}=-\left(\left\langle\sigma_{3} \xi_{j}, \partial_{\omega} R\right\rangle, \mathrm{i}\left\langle\sigma_{3} \xi_{j}, \sigma_{3} R\right\rangle\right)\left(\begin{array}{c}
\nabla \omega \\
\nabla \vartheta
\end{array}\right)+e^{-\mathrm{i} \sigma_{3} \vartheta} \sigma_{3} \xi_{j} .
$$

This yields (4.8). Similarly

$$
\nabla \bar{z}_{j}=a e^{-\mathrm{i} \sigma_{3} \vartheta} \Phi+b e^{-\mathrm{i} \sigma_{3} \vartheta} \sigma_{3} \partial_{\omega} \Phi+e^{-\mathrm{i} \sigma_{3} \vartheta} \sigma_{1} \sigma_{3} \xi_{j},
$$

where

$$
\mathcal{A}^{*}\left(\begin{array}{l}
a \\
b
\end{array}\right)=\left(\begin{array}{c}
\left\langle\sigma_{1} \sigma_{3} \xi_{j}, \partial_{\omega} R\right\rangle \\
\mathrm{i}\left\langle\sigma_{1} \sigma_{3} \xi_{j}, \sigma_{3} R\right\rangle
\end{array}\right)
$$

Lemma 4.2. Consider the map $f(U)=f$ for $U$ and $f$ as in (3.13). Denote by $f^{\prime}(U)$ the Frechét derivative of this map. Then

$$
f^{\prime}(U)=\left(P_{c}(\omega) P_{c}\left(\omega_{0}\right)\right)^{-1} P_{c}(\omega)\left[-\partial_{\omega} R d \omega-\mathrm{i} \sigma_{3} R d \vartheta+e^{-\mathrm{i} \sigma_{3} \vartheta} \mathbb{1}\right] .
$$

Proof. We have

$$
\begin{aligned}
& f^{\prime}(U) e^{\mathrm{i} \sigma_{3} \vartheta} \xi_{\ell} \equiv f^{\prime}(U) e^{\mathrm{i} \sigma_{3} \vartheta} \sigma_{1} \xi_{\ell} \equiv 0=f^{\prime}(U) e^{\mathrm{i} \sigma_{3} \vartheta} \sigma_{3}(\Phi+R)= \\
& \left.f^{\prime}(U) e^{\mathrm{i} \sigma_{3} \vartheta} \partial_{\omega}(\Phi+R)\right\rangle \text { and } f^{\prime}(U) e^{\mathrm{i} \sigma_{3} \vartheta} P_{c}(\omega) g=g \forall g \in L_{c}^{2}\left(\mathcal{H}_{\omega_{0}}\right) .
\end{aligned}
$$


This implies that for a pair of vectors valued functions $A$ and $B$ and with the inverse of $P_{c}\left(\mathcal{H}_{\omega}\right) P_{c}\left(\mathcal{H}_{\omega_{0}}\right): L_{c}^{2}\left(\mathcal{H}_{\omega_{0}}\right) \rightarrow L_{c}^{2}\left(\mathcal{H}_{\omega}\right)$,

$$
\begin{aligned}
& f^{\prime}=(A, B)\left(\begin{array}{c}
\left\langle e^{-\mathrm{i} \sigma_{3} \vartheta} \Phi,\right\rangle \\
\left\langle e^{-\mathrm{i} \sigma_{3} \vartheta} \sigma_{3} \partial_{\omega} \Phi,\right.
\end{array}\right)+\left(P_{c}(\omega) P_{c}\left(\omega_{0}\right)\right)^{-1} P_{c}(\omega) e^{-\mathrm{i} \sigma_{3} \vartheta}= \\
& -(A, B) \mathcal{A}\left(\begin{array}{c}
d \omega \\
d \vartheta
\end{array}\right)+\left(P_{c}(\omega) P_{c}\left(\omega_{0}\right)\right)^{-1} P_{c}(\omega) e^{-\mathrm{i} \sigma_{3} \vartheta} .
\end{aligned}
$$

By (4.11) we obtain that $A$ and $B$ are identified by the following equations (treating the last $\left(P_{c}(\omega) P_{c}\left(\omega_{0}\right)\right)^{-1} P_{c}(\omega)$ like a scalar):

$$
\mathcal{A}^{*}\left(\begin{array}{l}
A \\
B
\end{array}\right)=\left(P_{c}(\omega) P_{c}\left(\omega_{0}\right)\right)^{-1} P_{c}(\omega)\left(\begin{array}{c}
\partial_{\omega} R \\
\mathrm{i} \sigma_{3} R
\end{array}\right) .
$$

\section{Symplectic structure}

Our ambient space is $H^{1}\left(\mathbb{R}^{3}, \mathbb{C}\right) \times H^{1}\left(\mathbb{R}^{3}, \mathbb{C}\right)$. We focus only on points with $\sigma_{1} U=\bar{U}$. The natural symplectic structure for our problem is

$$
\Omega(X, Y)=\left\langle X, \sigma_{3} \sigma_{1} Y\right\rangle .
$$

We will see that the coordinates we introduced in (3.13), which arise naturally from the linearization, are not canonical for (5.1). This is the main difference with $[\mathrm{BC}]$. In this section we exploit the work in section 4 to compute the Poisson brackets for pairs of coordinates. We end the section with a crucial property for $Q$, Lemma 5.4.

The hamiltonian vector field $X_{G}$ of a scalar function $G$ is defined by the equation $\left\langle X_{G}, \sigma_{3} \sigma_{1} Y\right\rangle=-\mathrm{i}\langle\nabla G, Y\rangle$ for any vector $Y$ and is $X_{G}=-\mathrm{i} \sigma_{3} \sigma_{1} \nabla G$. At $U=e^{\mathrm{i} \sigma_{3} \vartheta}\left(\Phi_{\omega}+R\right)$ as in (3.12) we have by (4.5)

$$
\begin{aligned}
& X_{G}(U)=\mathrm{i} \frac{\left\langle\nabla G(U), e^{\mathrm{i} \sigma_{3} \vartheta} \sigma_{3} \Phi\right\rangle}{q^{\prime}(\omega)} e^{\mathrm{i} \sigma_{3} \vartheta} \partial_{\omega} \Phi-\mathrm{i} \frac{\left\langle\nabla G(U), e^{\mathrm{i} \sigma_{3} \vartheta} \partial_{\omega} \Phi\right\rangle}{q^{\prime}(\omega)} e^{\mathrm{i} \sigma_{3} \vartheta} \sigma_{3} \Phi \\
& +\mathrm{i} \sum_{j} \partial_{z_{j}} G(U) e^{\mathrm{i} \sigma_{3} \vartheta} \sigma_{1} \xi_{j}-\mathrm{i} \sum_{j} \partial_{\bar{z}_{j}} G(U) e^{\mathrm{i} \sigma_{3} \vartheta} \xi_{j}- \\
& -\mathrm{i} e^{\mathrm{i} \sigma_{3} \vartheta} \sigma_{3} \sigma_{1} P_{c}\left(\mathcal{H}_{\omega}^{*}\right) e^{\mathrm{i} \sigma_{3} \vartheta} \nabla G(U) .
\end{aligned}
$$

We call Poisson bracket of a pair of scalar valued functions $F$ and $G$ the scalar valued function

$$
\{F, G\}=\left\langle\nabla F, X_{G}\right\rangle=-\mathrm{i}\left\langle\nabla F, \sigma_{3} \sigma_{1} \nabla G\right\rangle=\mathrm{i} \Omega\left(X_{F}, X_{G}\right) .
$$

By $0=\mathrm{i} \frac{d}{d t} Q(U(t))=\left\langle\nabla Q(U(t)), \sigma_{3} \sigma_{1} \nabla E(U(t))\right\rangle$ we have the commutation

$$
\{Q, E\}=0 \text {. }
$$


In terms of spectral components we have

$$
\begin{aligned}
& \mathrm{i}\{F, G\}(U)=\left\langle\nabla F(U), \sigma_{3} \sigma_{1} \nabla G(U)\right\rangle=\left(q^{\prime}\right)^{-1} \times \\
& {\left[\left\langle\nabla F, e^{\mathrm{i} \sigma_{3} \vartheta} \sigma_{3} \Phi\right\rangle\left\langle\nabla G, e^{\mathrm{i} \sigma_{3} \vartheta} \partial_{\omega} \Phi\right\rangle-\left\langle\nabla F, e^{\mathrm{i} \sigma_{3} \vartheta} \partial_{\omega} \Phi\right\rangle\left\langle\nabla G, e^{\mathrm{i} \sigma_{3} \vartheta} \sigma_{3} \Phi\right\rangle\right]} \\
& +\sum_{j}\left[\partial_{z_{j}} F \partial_{\bar{z}_{j}} G-\partial_{\bar{z}_{j}} F \partial_{z_{j}} G\right]+ \\
& +\left\langle\sigma_{3} e^{-\mathrm{i} \sigma_{3} \vartheta} P_{c}\left(\mathcal{H}_{\omega}^{*}\right) e^{\mathrm{i} \sigma_{3} \vartheta} \nabla F, \sigma_{1} e^{-\mathrm{i} \sigma_{3} \vartheta} P_{c}\left(\mathcal{H}_{\omega}^{*}\right) e^{\mathrm{i} \sigma_{3} \vartheta} \nabla G\right\rangle .
\end{aligned}
$$

Lemma 5.1. Let $F(U)$ be a scalar function. We have the following equalities:

$$
\begin{gathered}
\{\omega, \vartheta\}=\frac{q^{\prime}}{\left(q^{\prime}\right)^{2}-\left\langle R, \partial_{\omega} \Phi\right\rangle^{2}+\left\langle\sigma_{3} R, \Phi\right\rangle\left\langle R, \sigma_{3} \partial_{\omega}^{2} \Phi\right\rangle} \\
\left\{z_{j}, F\right\}=\left\langle\sigma_{3} \xi_{j}, \partial_{\omega} R\right\rangle\{F, \omega\}+\mathrm{i}\left\langle\sigma_{3} \xi_{j}, \sigma_{3} R\right\rangle\{F, \vartheta\}-\mathrm{i} \partial_{z_{j}} F \\
\left\{\bar{z}_{j}, F\right\}=\left\langle\sigma_{1} \sigma_{3} \xi_{j}, \partial_{\omega} R\right\rangle\{F, \omega\}+\mathrm{i}\left\langle\sigma_{1} \sigma_{3} \xi_{j}, \sigma_{3} R\right\rangle\{F, \vartheta\}+\mathrm{i} \partial_{z_{j}} F .
\end{gathered}
$$

In particular we have:

$$
\begin{aligned}
& \left\{z_{j}, \omega\right\}=\mathrm{i}\left\langle\sigma_{3} \xi_{j}, \sigma_{3} R\right\rangle\{\omega, \vartheta\} ;\left\{\bar{z}_{j}, \omega\right\}=\mathrm{i}\left\langle\sigma_{1} \sigma_{3} \xi_{j}, \sigma_{3} R\right\rangle\{\omega, \vartheta\} ; \\
& \left\{z_{j}, \vartheta\right\}=\left\langle\sigma_{3} \xi_{j}, \partial_{\omega} R\right\rangle\{\vartheta, \omega\} ;\left\{\bar{z}_{j}, \vartheta\right\}=\left\langle\sigma_{1} \sigma_{3} \xi_{j}, \partial_{\omega} R\right\rangle\{\vartheta, \omega\} ; \\
& \left\{z_{k}, z_{j}\right\}=\mathrm{i}\left(\left\langle\sigma_{3} \xi_{k}, \partial_{\omega} R\right\rangle\left\langle\sigma_{3} \xi_{j}, \sigma_{3} R\right\rangle-\left\langle\sigma_{3} \xi_{j}, \partial_{\omega} R\right\rangle\left\langle\sigma_{3} \xi_{k}, \sigma_{3} R\right\rangle\right)\{\omega, \vartheta\} ; \\
& \left\{\bar{z}_{k}, \bar{z}_{j}\right\}=\mathrm{i}\left(\left\langle\sigma_{1} \sigma_{3} \xi_{k}, \partial_{\omega} R\right\rangle\left\langle\sigma_{1} \sigma_{3} \xi_{j}, \sigma_{3} R\right\rangle-\left\langle\sigma_{1} \sigma_{3} \xi_{j}, \partial_{\omega} R\right\rangle\left\langle\sigma_{1} \sigma_{3} \xi_{k}, \sigma_{3} R\right\rangle\right)\{\omega, \vartheta\} ; \\
& \left\{z_{k}, \bar{z}_{j}\right\}=-\mathrm{i} \delta_{j k}+\mathrm{i}\left(\left\langle\sigma_{3} \xi_{k}, \partial_{\omega} R\right\rangle\left\langle\sigma_{1} \sigma_{3} \xi_{j}, \sigma_{3} R\right\rangle-\left\langle\sigma_{1} \sigma_{3} \xi_{j}, \partial_{\omega} R\right\rangle\left\langle\xi_{k}, R\right\rangle\right)\{\omega, \vartheta\} .
\end{aligned}
$$

Proof. By (4.3) and (5.5) we have i $\{\omega, \vartheta\}=$

$$
\begin{aligned}
& \left(q^{\prime}\right)^{-1}\left[\left\langle\nabla \omega, e^{\mathrm{i} \sigma_{3} \vartheta} \sigma_{3} \Phi\right\rangle\left\langle\nabla \vartheta, e^{\mathrm{i} \sigma_{3} \vartheta} \partial_{\omega} \Phi\right\rangle-\left\langle\nabla \omega, e^{\mathrm{i} \sigma_{3} \vartheta} \partial_{\omega} \Phi\right\rangle\left\langle\nabla \vartheta, e^{\mathrm{i} \sigma_{3} \vartheta} \sigma_{3} \Phi\right\rangle\right]= \\
& \frac{-\left\langle\sigma_{3} R, \Phi_{\omega}\right\rangle q^{\prime}\left\langle R, \sigma_{3} \partial_{\omega}^{2} \Phi_{\omega}\right\rangle q^{\prime}-\left[\left(q^{\prime}(\omega)\right)^{2}-\left\langle R, \partial_{\omega} \Phi_{\omega}\right\rangle^{2}\right]\left(q^{\prime}\right)^{2}}{\left.q^{\prime} \mathrm{i}\left[q^{\prime}(\omega)\right)^{2}-\left\langle R, \partial_{\omega} \Phi_{\omega}\right\rangle^{2}+\left\langle\sigma_{3} R, \Phi_{\omega}\right\rangle\left\langle R, \sigma_{3} \partial_{\omega}^{2} \Phi_{\omega}\right\rangle\right]^{2}} .
\end{aligned}
$$

This yields (5.6). For (5.7), substituting (4.8) in (5.3), we get $\left\{z_{j}, F\right\}=$

$$
\left\langle\nabla z_{j}, X_{F}\right\rangle=-\left\langle\sigma_{3} \xi_{j}, \partial_{\omega} R\right\rangle\{\omega, F\}-\mathrm{i}\left\langle\sigma_{3} \xi_{j}, \sigma_{3} R\right\rangle\{\vartheta, F\}+\left\langle e^{-\mathrm{i} \sigma_{3} \vartheta} \sigma_{3} \xi_{j}, X_{F}\right\rangle .
$$

When we substitute $X_{F}$ with the decomposition in (5.2), the last term in the above sum becomes $\left\langle e^{-\mathrm{i} \sigma_{3} \vartheta} \sigma_{3} \xi_{j}, X_{F}\right\rangle=-\mathrm{i} \partial_{\bar{z}_{j}} F\left\langle e^{-\mathrm{i} \sigma_{3} \vartheta} \sigma_{3} \xi_{j}, e^{\mathrm{i} \sigma_{3} \vartheta} \xi_{j}\right\rangle=-\mathrm{i} \partial_{\bar{z}_{j}} F$. This yields (5.7). (5.8) can be derived by first replacing $F$ with $\bar{F}$ in (5.7) and by taking the complex conjugate of the resulting equation:

$$
\left\{z_{j}, F\right\}=\left\langle\sigma_{3} \xi_{j}, \partial_{\omega} \bar{R}\right\rangle\{F, \omega\}-\mathrm{i}\left\langle\sigma_{3} \xi_{j}, \sigma_{3} \bar{R}\right\rangle\{F, \vartheta\}+\mathrm{i} \partial_{z_{j}} F .
$$

Then (5.8) follows by using that $\bar{R}=\sigma_{1} R$ and $\sigma_{1} \sigma_{3}=-\sigma_{3} \sigma_{1}$. The remaining formulas in the statement follow from $(5.7)-(5.8)$.

Definition 5.2. Given a function $\mathcal{G}(U)$ with values in $L_{c}^{2}\left(\mathcal{H}_{\omega_{0}}\right)$, a symplectic form $\Omega$ and a scalar function $F(U)$, we define

$$
\{\mathcal{G}, F\}:=\mathcal{G}^{\prime}(U) X_{F}(U)
$$

with $X_{F}$ the hamiltonian vector field associated to $F$. We set $\{F, \mathcal{G}\}:=-\{\mathcal{G}, F\}$. 
We have:

Lemma 5.3. For $f(U)$ the functional in Lemma 4.2, we have:

$$
\{f, F\}=\left(P_{c}(\omega) P_{c}\left(\omega_{0}\right)\right)^{-1} P_{c}(\omega)\left[\{F, \omega\} \partial_{\omega} R+\mathrm{i}\{F, \vartheta\} \sigma_{3} R-\mathrm{i} e^{-\mathrm{i} \sigma_{3} \vartheta} \sigma_{3} \sigma_{1} \nabla F\right] .
$$

In particular we have:

$$
\begin{aligned}
& \{f, \omega\}=\mathrm{i}\{\omega, \vartheta\}\left(P_{c}(\omega) P_{c}\left(\omega_{0}\right)\right)^{-1} P_{c}(\omega) \sigma_{3} R ; \\
& \{f, \vartheta\}=\{\vartheta, \omega\}\left(P_{c}(\omega) P_{c}\left(\omega_{0}\right)\right)^{-1} P_{c}(\omega) \partial_{\omega} R ; \\
& \left\{f, z_{j}\right\}=\left(P_{c}(\omega) P_{c}\left(\omega_{0}\right)\right)^{-1} P_{c}(\omega)\left[\left\{z_{j}, \omega\right\} \partial_{\omega} R+\mathrm{i}\left\{z_{j}, \vartheta\right\} \sigma_{3} R\right] ; \\
& \left\{f, \bar{z}_{j}\right\}=\left(P_{c}(\omega) P_{c}\left(\omega_{0}\right)\right)^{-1} P_{c}(\omega)\left[\left\{\bar{z}_{j}, \omega\right\} \partial_{\omega} R+\mathrm{i}\left\{\bar{z}_{j}, \vartheta\right\} \sigma_{3} R\right] .
\end{aligned}
$$

Proof. Using Lemma 4.2 and by (4.2)

$$
\begin{aligned}
& f^{\prime} \sigma_{3} \sigma_{1} \nabla F=-(A, B) \mathcal{A}\left(\begin{array}{l}
\left\langle\nabla \omega, \sigma_{3} \sigma_{1} \nabla F\right\rangle \\
\left\langle\nabla \vartheta, \sigma_{3} \sigma_{1} \nabla F\right\rangle
\end{array}\right) \\
& +\left(P_{c}(\omega) P_{c}\left(\omega_{0}\right)\right)^{-1} P_{c}(\omega) e^{-\mathrm{i} \sigma_{3} \vartheta} \sigma_{3} \sigma_{1} \nabla F .
\end{aligned}
$$

By Lemma 4.2 we have

$$
(A, B) \mathcal{A}\left(\begin{array}{l}
\{\omega, F\} \\
\{\vartheta, F\}
\end{array}\right)=\left(P_{c}(\omega) P_{c}\left(\omega_{0}\right)\right)^{-1} P_{c}(\omega)\left(\partial_{\omega} R, \mathrm{i} \sigma_{3} R\right)\left(\begin{array}{l}
\{\omega, F\} \\
\{\vartheta, F\}
\end{array}\right) .
$$

The following result is important in the sequel.

Lemma 5.4. Let $Q$ be the function defined in (3.3). Then, we have the following formulas:

$$
\begin{gathered}
\{Q, \omega\}=0 ; \\
\{Q, \vartheta\}=1 ; \\
\left\{Q, z_{j}\right\}=\left\{Q, \bar{z}_{j}\right\}=0 ; \\
\{Q, f\}=0 .
\end{gathered}
$$

Denote by $X_{Q}$ the hamiltonian vectorfield of $Q$. Then

$$
X_{Q}=-\frac{\partial}{\partial \vartheta}
$$

Proof. We have by (5.5), (4.3) and $\nabla Q(U)=\sigma_{1} U$,

$$
\begin{aligned}
& \mathrm{i} q^{\prime}\{Q, \omega\}=\left\langle\nabla Q, e^{\mathrm{i} \sigma_{3} \vartheta} \sigma_{3} \Phi\right\rangle\left\langle\nabla \omega, e^{\mathrm{i} \sigma_{3} \vartheta} \partial_{\omega} \Phi\right\rangle-\left\langle\nabla Q, e^{\mathrm{i} \sigma_{3} \vartheta} \partial_{\omega} \Phi\right\rangle\left\langle\nabla \omega, e^{\mathrm{i} \sigma_{3} \vartheta} \sigma_{3} \Phi\right\rangle \\
& =q^{\prime} \frac{-\left\langle R, \sigma_{3} \Phi\right\rangle\left(q^{\prime}(\omega)+\left\langle R, \partial_{\omega} \Phi_{\omega}\right\rangle\right)-\left(q^{\prime}(\omega)+\left\langle R, \partial_{\omega} \Phi_{\omega}\right\rangle\right)(-1)\left\langle R, \sigma_{3} \Phi\right\rangle}{\left(q^{\prime}(\omega)\right)^{2}-\left\langle R, \partial_{\omega} \Phi_{\omega}\right\rangle^{2}+\left\langle\sigma_{3} R, \Phi_{\omega}\right\rangle\left\langle R, \sigma_{3} \partial_{\omega}^{2} \Phi_{\omega}\right\rangle}=0
\end{aligned}
$$


Similarly,

$$
\begin{aligned}
& \mathrm{i} q^{\prime}\{Q, \vartheta\}=\left\langle\nabla Q, e^{\mathrm{i} \sigma_{3} \vartheta} \sigma_{3} \Phi\right\rangle\left\langle\nabla \vartheta, e^{\mathrm{i} \sigma_{3} \vartheta} \partial_{\omega} \Phi\right\rangle-\left\langle\nabla Q, e^{\mathrm{i} \sigma_{3} \vartheta} \partial_{\omega} \Phi\right\rangle\left\langle\nabla \vartheta, e^{\mathrm{i} \sigma_{3} \vartheta} \sigma_{3} \Phi\right\rangle \\
& =q^{\prime} \frac{-\left\langle R, \sigma_{3} \Phi\right\rangle\left\langle R, \sigma_{3} \partial_{\omega}^{2} \Phi\right\rangle-\left(q^{\prime}(\omega)+\left\langle R, \partial_{\omega} \Phi_{\omega}\right\rangle\right)\left(q^{\prime}(\omega)-\left\langle R, \partial_{\omega} \Phi_{\omega}\right\rangle\right)}{\mathrm{i}\left[\left(q^{\prime}(\omega)\right)^{2}-\left\langle R, \partial_{\omega} \Phi_{\omega}\right\rangle^{2}+\left\langle\sigma_{3} R, \Phi_{\omega}\right\rangle\left\langle R, \sigma_{3} \partial_{\omega}^{2} \Phi_{\omega}\right\rangle\right]}=q^{\prime} \mathrm{i} .
\end{aligned}
$$

By (5.7),(5.12) and (5.13) we have

$$
\begin{aligned}
& \mathrm{i}\left\{z_{j}, Q\right\}=-\left\langle\xi_{j}, R\right\rangle+\partial_{\bar{z}_{j}} Q \\
& \mathrm{i}\left\{\bar{z}_{j}, Q\right\}=\left\langle\xi_{j}, \sigma_{1} R\right\rangle-\partial_{z_{j}} Q .
\end{aligned}
$$

By

$$
Q(U)=q+\frac{1}{2}\left\langle z \cdot \xi+\bar{z} \cdot \sigma_{1} \xi+P_{c}(\omega) f, \sigma_{1}\left(z \cdot \xi+\bar{z} \cdot \sigma_{1} \xi+P_{c}(\omega) f\right)\right\rangle
$$

we have

$$
\partial_{z_{j}} Q=\left\langle\xi_{j}, \sigma_{1} R\right\rangle, \quad \partial_{\bar{z}_{j}} Q=\left\langle\xi_{j}, R\right\rangle .
$$

So both lines in (5.17) are 0 and yield (5.14). Finally (5.15) follows by (5.9), Lemma 5.3, (5.12), (5.13) and by

$$
\begin{aligned}
& \{f, Q\}=\left(P_{c}(\omega) P_{c}\left(\omega_{0}\right)\right)^{-1} P_{c}(\omega)\left[\mathrm{i}\{Q, \vartheta\} \sigma_{3} R-\mathrm{i} e^{-\mathrm{i} \sigma_{3} \vartheta} \sigma_{3} \sigma_{1} \nabla Q\right] \\
& =\left(P_{c}(\omega) P_{c}\left(\omega_{0}\right)\right)^{-1} P_{c}(\omega)\left[\mathrm{i} \sigma_{3} R-\mathrm{i} \sigma_{3} \Phi-\mathrm{i} \sigma_{3} R\right]=0 .
\end{aligned}
$$

(5.16) is an immediate consequence of the definition of $X_{Q}$ and of (5.12)-(5.15).

\section{Hamiltonian riformulation of the system}

(3.20) is how the problem is framed in the literature. Yet (3.20) hides the crucial hamiltonian nature of the problem. In the coordinate system (3.13) can be written as follows:

$$
\begin{aligned}
& \dot{\omega}=\{\omega, E\}, \quad \dot{f}=\{f, E\}, \\
& \dot{z}_{j}=\left\{z_{j}, E\right\}, \quad \dot{\bar{z}}_{j}=\left\{\bar{z}_{j}, E\right\}, \\
& \dot{\vartheta}=\{\vartheta, E\} .
\end{aligned}
$$

For the scalar coordinates the equations in (6.1) are due to the hamiltonian nature of (3.5). Exactly for the same reasons we have the equation $\dot{f}=\{f, E\}$, which we now derive in the following standard way. Multiplying (3.20) by $e^{\mathrm{i} \vartheta \sigma_{3}}$ one can rewrite (3.20) by (4.6) and (3.6), as

$$
\begin{aligned}
& -\mathrm{i} \dot{\vartheta} \frac{\partial}{\partial \vartheta}+\mathrm{i} \dot{\omega} \frac{\partial}{\partial \omega}+\mathrm{i} \sum_{j} \dot{z}_{j} \frac{\partial}{\partial z_{j}}+\mathrm{i} \sum_{j} \dot{\bar{z}}_{j} \frac{\partial}{\partial \bar{z}_{j}} \\
& +\mathrm{i} e^{\mathrm{i} \vartheta \sigma_{3}} P_{c}\left(\mathcal{H}_{\omega}\right) \dot{f}=\sigma_{3} \sigma_{1} \nabla E(U) .
\end{aligned}
$$


When we apply the derivative $f^{\prime}(U)$ to $(6.2)$ the first line cancels, so that

$$
\dot{f}=f^{\prime}(U) e^{\mathrm{i} \vartheta \sigma_{3}} P_{c}\left(\mathcal{H}_{\omega}\right) \dot{f}=-f^{\prime}(U) \mathrm{i} \sigma_{3} \sigma_{1} \nabla E(U)=f^{\prime}(U) X_{E}(U)=\{f, E\} .
$$

where the first equality is (4.11), the third the definition of hamiltonian field two lines above (5.2) and the last equality is Definition 5.2.

We now introduce a new hamiltonian. For $u_{0}$ the initial datum in (1.1), set

$$
K(U)=E(U)+\omega(U) Q(U)-\omega(U)\left\|u_{0}\right\|_{L_{x}^{2}}^{2} .
$$

By Lemma 5.4 the solution of the initial value problem in (1.1) solves also

$$
\begin{aligned}
& \dot{\omega}=\{\omega, K\}, \quad \dot{f}=\{f, K\}, \\
& \dot{z}_{j}=\left\{z_{j}, K\right\}, \quad \dot{\bar{z}}_{j}=\left\{\bar{z}_{j}, K\right\}, \\
& \dot{\vartheta}-\omega=\{\vartheta, K\} .
\end{aligned}
$$

By $\frac{\partial}{\partial \vartheta} K=0$ the right hand sides in the equations (6.4) do not depend on $\vartheta$. Hence, if we look at the new system

$$
\begin{aligned}
& \dot{\omega}=\{\omega, K\}, \quad \dot{f}=\{f, K\}, \\
& \dot{z}_{j}=\left\{z_{j}, K\right\}, \quad \dot{\bar{z}}_{j}=\left\{\bar{z}_{j}, K\right\}, \\
& \dot{\vartheta}=\{\vartheta, K\},
\end{aligned}
$$

the evolution of the crucial variables $(\omega, z, \bar{z}, f)$ in (6.1) and (6.5) is the same. Therefore, to prove Theorem 2.2 it is sufficient to consider system (6.5).

\section{Application of the Darboux Theorem}

Since the main obstacle at reproducing the Birkhoff normal forms argument of $[\mathrm{BC}]$ for (6.5) is that the coordinates (3.13) are not canonical, we change coordinates. That is, we apply the Darboux Theorem. We warn the reader not to confuse the variable $t \in[0,1]$ of this section with the time of the evolution equation of the other sections.

We introduce the 2-form, for $q=q(\omega)=\left\|\phi_{\omega}\right\|_{L_{x}^{2}}^{2}$ and summing on repeated indexes,

$$
\Omega_{0}=\mathrm{i} d \vartheta \wedge d q+d z_{j} \wedge d \bar{z}_{j}+\left\langle f^{\prime}(U) \quad, \sigma_{3} \sigma_{1} f^{\prime}(U) \quad\right\rangle,
$$

with $f(U)$ the function in Lemma $4.2, f^{\prime}(U)$ its Frechét derivative and the last term in (7.1) acting on pairs $(X, Y)$ like $\left\langle f^{\prime}(U) X, \sigma_{3} \sigma_{1} f^{\prime}(U) Y\right\rangle$. It is an elementary exercise to show that $\Omega_{0}$ is a closed and non degenerate 2 form. In Lemma 7.1 we check that $\Omega_{0}(U)=\Omega(U)$ at $U=e^{\mathrm{i} \sigma_{3} \vartheta} \Phi_{\omega_{0}}$. Then the proof of the Darboux Theorem goes as follows. One first considers

$$
\Omega_{t}=(1-t) \Omega_{0}+t \Omega=\Omega_{0}+t \widetilde{\Omega} \text { with } \widetilde{\Omega}:=\Omega-\Omega_{0} .
$$

Then one considers a 1- differential form $\gamma(t, U)$ such that (external differentiation will always be on the $U$ variable only) i $d \gamma(t, U)=\widetilde{\Omega}$ with $\gamma(U)=0$ at 
$U=e^{\mathrm{i} \sigma_{3} \vartheta} \Phi_{\omega_{0}}$. Finally one considers the vector field $\mathcal{Y}^{t}$ such that $i_{\mathcal{Y}^{t}} \Omega_{t}=-\mathrm{i} \gamma$ (here for $\Omega$ a 2 form and $Y$ a vector field, $i_{Y} \Omega$ is the 1 form defined by $\left.i_{Y} \Omega(X):=\Omega(Y, X)\right)$ and the flow $\mathfrak{F}_{t}$ generated by $\mathcal{Y}^{t}$, which near the points $e^{\mathrm{i} \sigma_{3} \vartheta} \Phi_{\omega_{0}}$ is defined up to time 1 , and show that $\mathfrak{F}_{1}^{*} \Omega=\Omega_{0}$ by

$$
\begin{aligned}
& \frac{d}{d t}\left(\mathfrak{F}_{t}^{*} \Omega_{t}\right)=\mathfrak{F}_{t}^{*}\left(L_{\mathcal{Y}_{t}} \Omega_{t}\right)+\mathfrak{F}_{t}^{*} \frac{d}{d t} \Omega_{t}= \\
& =\mathfrak{F}_{t}^{*} d\left(i_{\mathcal{Y}^{t}} \Omega_{t}\right)+\mathfrak{F}_{t}^{*} \widetilde{\Omega}=\mathfrak{F}_{t}^{*}(-\mathrm{i} d \gamma+\widetilde{\Omega})=0 .
\end{aligned}
$$

For $\Omega_{0}$, the coordinates (3.13) are canonical. But if one does not choose the 1 form $\gamma$ carefully, then the new hamiltonian $\widetilde{K}=K \circ \mathfrak{F}_{1}$ will not yield a semilinear NLS for coordinates (3.13), which is what we need to perform the argument of $[\mathrm{BC}, \mathrm{CM}]$. In the sequel of this section all the work is finalized to the correct choice if $\gamma$. In Lemma 7.2 we compute explicitly a differential form $\alpha$ and we make the preliminary choice $\gamma=-\mathrm{i} \alpha$. This is not yet the right choice. By the computations in Lemma 7.3 and Remark 7.4, we find the obstruction to the fact that $\widetilde{K}$ is of the desired type. Lemmas $7.5-7.7$ are necessary to find an appropriate solution $F$ of a differential equation in Lemma 7.8. Then $\gamma=-\mathrm{i} \alpha+d F$ is the right choice of $\gamma$. In Lemma 7.10 we collect a number of useful estimates for $\mathfrak{F}_{1}$. Finally, Lemma 7.11 is valid independently of the precise $\gamma$ chosen and contains information necessary for (8.1)-(8.2).

For any vector $Y \in T_{U} L^{2}$ we set

$$
Y=Y_{\vartheta} \frac{\partial}{\partial \vartheta}+Y_{\omega} \frac{\partial}{\partial \omega}+\sum Y_{j} \frac{\partial}{\partial z_{j}}+\sum Y_{\bar{j}} \frac{\partial}{\partial \bar{z}_{j}}+e^{\mathrm{i} \sigma_{3} \vartheta} P_{c}(\omega) Y_{f}
$$

for

$$
\begin{gathered}
Y_{\vartheta}=d \vartheta(Y), \quad Y_{\omega}=d \omega(Y), \quad Y_{j}=d z_{j}(Y) \\
Y_{\bar{j}}=d \bar{z}_{j}(Y), \quad Y_{f}=f^{\prime}(U) Y .
\end{gathered}
$$

Similarly, a differential 1-form $\gamma$ decomposes as

$$
\gamma=\gamma^{\vartheta} d \vartheta+\gamma^{\omega} d \omega+\sum \gamma^{j} d z_{j}+\sum \gamma^{j} d \bar{z}_{j}+\left\langle\gamma^{f}, f^{\prime} \quad\right\rangle,
$$

where: $\left\langle\gamma^{f}, f^{\prime}\right\rangle$ acts on a vector $Y$ as $\left\langle\gamma^{f}, f^{\prime} Y\right\rangle$, with here $\gamma^{f} \in L_{c}^{2}\left(\mathcal{H}_{\omega_{0}}^{*}\right) ; \gamma^{\vartheta}$, $\gamma^{\omega}, \gamma^{j}$ and $\gamma^{\bar{j}}$ are in $\mathbb{C}$. Notice that we are reversing the standard notation on super and subscripts for forms and vector fields. In the sequel, given a differential 1 form $\gamma$ and a point $U$, we will denote by $\gamma_{U}$ the value of $\gamma$ at $U$.

Given a function $\chi$, denote its hamiltonian vector field with respect to $\Omega_{t}$ by $X_{\chi}^{t}: i_{X_{\chi}^{t}} \Omega_{t}=-\mathrm{i} d \chi$. By (7.1) we have the following hamiltonian vectorfield associated to $q(\omega)$ (this is important in Lemma 7.11 later):

$$
X_{q(\omega)}^{0}=-\frac{\partial}{\partial \vartheta} .
$$

We have the following preliminary observation:

Lemma 7.1. At $U=e^{\mathrm{i} \sigma_{3} \vartheta} \Phi_{\omega_{0}}$, for any $\vartheta$, we have $\Omega_{0}(U)=\Omega(U)$. 
Proof. Using the following partition of the identity

$$
\mathbb{1}=e^{\mathrm{i} \sigma_{3} \vartheta}\left[P_{N_{g}\left(\mathcal{H}_{\omega}\right)}+\sum_{\lambda \in \sigma\left(\mathcal{H}_{\omega}\right) \backslash\{0\}} P_{\operatorname{ker}\left(\mathcal{H}_{\omega}-\lambda\right)}+P_{c}\left(\mathcal{H}_{\omega}\right)\right] e^{-\mathrm{i} \sigma_{3} \vartheta}
$$

we get, summing on repeated indexes,

$$
\begin{aligned}
& \Omega(X, Y)=\left\langle X, \sigma_{3} \sigma_{1} Y\right\rangle= \\
& \frac{1}{q^{\prime}}\left[\left\langle X, e^{-\mathrm{i} \sigma_{3} \vartheta} \sigma_{3} \partial_{\omega} \Phi\right\rangle\left\langle Y, e^{-\mathrm{i} \sigma_{3} \vartheta} \Phi\right\rangle-\left\langle X, e^{-\mathrm{i} \sigma_{3} \vartheta} \Phi\right\rangle\left\langle Y, e^{-\mathrm{i} \sigma_{3} \vartheta} \sigma_{3} \partial_{\omega} \Phi\right\rangle\right]+ \\
& {\left[\left\langle X, e^{-\mathrm{i} \sigma_{3} \vartheta} \sigma_{3} \xi_{j}\right\rangle\left\langle Y, e^{-\mathrm{i} \sigma_{3} \vartheta} \sigma_{1} \sigma_{3} \xi_{j}\right\rangle-\left\langle X, e^{-\mathrm{i} \sigma_{3} \vartheta} \sigma_{1} \sigma_{3} \xi_{j}\right\rangle\left\langle Y, e^{-\mathrm{i} \sigma_{3} \vartheta} \sigma_{3} \xi_{j}\right\rangle\right]} \\
& +\left\langle P_{c}\left(\mathcal{H}_{\omega}\right) e^{-\mathrm{i} \sigma_{3} \vartheta} X, \sigma_{3} \sigma_{1} P_{c}\left(\mathcal{H}_{\omega}\right) e^{-\mathrm{i} \sigma_{3} \vartheta} Y\right\rangle .
\end{aligned}
$$

By (4.2) we have

$$
\left\langle, e^{-\mathrm{i} \sigma_{3} \vartheta} \sigma_{3} \partial_{\omega} \Phi\right\rangle \wedge\left\langle, e^{-\mathrm{i} \sigma_{3} \vartheta} \Phi\right\rangle=\operatorname{det} \mathcal{A} d \omega \wedge d \vartheta .
$$

Substituting (4.8)-(4.9) we get

$$
\begin{aligned}
& \left\langle, e^{-\mathrm{i} \sigma_{3} \vartheta} \sigma_{3} \xi_{j}\right\rangle \wedge\left\langle, e^{-\mathrm{i} \sigma_{3} \vartheta} \sigma_{1} \sigma_{3} \xi_{j}\right\rangle= \\
& \left(d z_{j}+\left\langle\sigma_{3} \xi_{j}, \partial_{\omega} R\right\rangle d \omega+\mathrm{i}\left\langle\sigma_{3} \xi_{j}, \sigma_{3} R\right\rangle d \vartheta\right) \\
& \wedge\left(d \bar{z}_{j}+\left\langle\sigma_{1} \sigma_{3} \xi_{j}, \partial_{\omega} R\right\rangle d \omega+\mathrm{i}\left\langle\sigma_{1} \sigma_{3} \xi_{j}, \sigma_{3} R\right\rangle d \vartheta\right) .
\end{aligned}
$$

By Lemma 4.2 we have

$$
\begin{aligned}
& \left\langle P_{c}\left(\mathcal{H}_{\omega}\right) e^{-\mathrm{i} \sigma_{3} \vartheta} \quad, \sigma_{3} \sigma_{1} P_{c}\left(\mathcal{H}_{\omega}\right) e^{-\mathrm{i} \sigma_{3} \vartheta} \quad\right\rangle= \\
& \left\langle P_{c}(\omega) P_{c}\left(\omega_{0}\right) f^{\prime} \quad+P_{c}(\omega) \partial_{\omega} R d \omega+\mathrm{i} P_{c}(\omega) \sigma_{3} R d \vartheta\right. \\
& \left.\sigma_{3} \sigma_{1}\left(P_{c}(\omega) P_{c}\left(\omega_{0}\right) f^{\prime} \quad+P_{c}(\omega) \partial_{\omega} R d \omega+\mathrm{i} P_{c}(\omega) \sigma_{3} R d \vartheta\right)\right\rangle .
\end{aligned}
$$

Then by (7.9)-(7.12) we have

$$
\begin{aligned}
& \Omega=\left(\mathrm{i} q^{\prime}+a_{1}\right) d \vartheta \wedge d \omega+d z_{j} \wedge d \bar{z}_{j}+ \\
& +d z_{j} \wedge\left(\left\langle\sigma_{1} \sigma_{3} \xi_{j}, \partial_{\omega} R\right\rangle d \omega+\mathrm{i}\left\langle\sigma_{1} \sigma_{3} \xi_{j}, \sigma_{3} R\right\rangle d \vartheta\right) \\
& -d \bar{z}_{j} \wedge\left(\left\langle\sigma_{3} \xi_{j}, \partial_{\omega} R\right\rangle d \omega+\mathrm{i}\left\langle\sigma_{3} \xi_{j}, \sigma_{3} R\right\rangle d \vartheta\right)+ \\
& +\left\langle P_{c}(\omega) P_{c}\left(\omega_{0}\right) f^{\prime} \quad, \sigma_{3} \sigma_{1} P_{c}(\omega) P_{c}\left(\omega_{0}\right) f^{\prime} \quad\right\rangle+ \\
& +\left\langle P_{c}(\omega) P_{c}\left(\omega_{0}\right) f^{\prime} \quad, \sigma_{3} \sigma_{1} P_{c}(\omega) \partial_{\omega} R\right\rangle \wedge d \omega+ \\
& +\mathrm{i}\left\langle P_{c}(\omega) P_{c}\left(\omega_{0}\right) f^{\prime} \quad, \sigma_{3} \sigma_{1} P_{c}(\omega) \sigma_{3} R\right\rangle \wedge d \vartheta
\end{aligned}
$$

where

$$
\begin{aligned}
& \mathrm{i} q^{\prime}+a_{1}=\frac{\operatorname{det} \mathcal{A}}{q^{\prime}}+\left\langle P_{c}(\omega) \partial_{\omega} R, \sigma_{3} \sigma_{1} P_{c}(\omega) \sigma_{3} \mathrm{i} R\right\rangle \\
& \quad+\left\langle\sigma_{3} \xi_{j}, \partial_{\omega} R\right\rangle\left\langle\sigma_{1} \sigma_{3} \xi_{j}, \mathrm{i} \sigma_{3} R\right\rangle-\left\langle\sigma_{1} \sigma_{3} \xi_{j}, \partial_{\omega} R\right\rangle\left\langle\sigma_{3} \xi_{j}, \mathrm{i} \sigma_{3} R\right\rangle .
\end{aligned}
$$

In particular we have

$$
a_{1}:=-\mathrm{i} q^{\prime}+\frac{\operatorname{det} \mathcal{A}}{q^{\prime}}+\left\langle P_{N_{g}^{\perp}\left(\mathcal{H}_{\omega}^{*}\right)} \mathrm{i} \sigma_{3} R, \sigma_{3} \sigma_{1} \partial_{\omega} R\right\rangle .
$$


Notice that $a_{1}=a_{1}(\omega, z, f)$ is smooth in the arguments $\omega \in \mathcal{O}, z \in \mathbb{C}^{n}$ and $f \in H^{-K^{\prime},-S^{\prime}}$ for any pair $\left(K^{\prime}, S^{\prime}\right)$ with, for $(z, f)$ near 0 ,

$$
\left|a_{1}\right| \leq C\left(K^{\prime}, S^{\prime}\right)\left(|z|+\|f\|_{H^{-K^{\prime},-S^{\prime}}}\right)^{2} .
$$

At points $U=e^{\mathrm{i} \sigma_{3} \vartheta} \Phi_{\omega}$, that is for $R=0$, we have

$$
\Omega=\mathrm{i} d \vartheta \wedge d q+d z_{j} \wedge d \bar{z}_{j}+\left\langle P_{c}(\omega) P_{c}\left(\omega_{0}\right) f^{\prime} \quad, \sigma_{3} \sigma_{1} P_{c}(\omega) P_{c}\left(\omega_{0}\right) f^{\prime} \quad\right\rangle .
$$

At $\omega=\omega_{0}$ we get $\Omega=\Omega_{0}$.

Lemma 7.2. Consider the following forms:

$$
\begin{aligned}
& \beta(U) Y:=\frac{1}{2}\left\langle\sigma_{1} \sigma_{3} U, Y\right\rangle \\
& \beta_{0}(U):=-\mathrm{i} q d \vartheta-\sum_{j} \frac{\bar{z}_{j} d z_{j}-z_{j} d \bar{z}_{j}}{2}+\frac{1}{2}\left\langle f(U), \sigma_{3} \sigma_{1} f^{\prime}(U) \quad\right\rangle .
\end{aligned}
$$

Then

$$
d \beta_{0}=\Omega_{0}, \quad d \beta=\Omega .
$$

Set

$$
\alpha(U)=\beta(U)-\beta_{0}(U)+d \psi(U) \text { where } \psi(U):=\frac{1}{2}\left\langle\sigma_{3} \Phi, R\right\rangle .
$$

We have $\alpha=\alpha^{\vartheta} d \vartheta+\alpha^{\omega} d \omega+\left\langle\alpha^{f}, f^{\prime}\right\rangle$ with:

$$
\begin{aligned}
\alpha^{\vartheta}+\frac{\mathrm{i}}{2}\|f\|_{2}^{2}= & -\frac{\mathrm{i}}{2}\left\|z \cdot \xi+\bar{z} \cdot \sigma_{1} \xi\right\|_{2}^{2}-\mathrm{i}\left\langle z \cdot \xi+\bar{z} \cdot \sigma_{1} \xi, \sigma_{1} P_{c}(\omega) f\right\rangle \\
& -\frac{\mathrm{i}}{2}\left\langle\left(P_{c}(\omega)-P_{c}\left(\omega_{0}\right)\right) f, \sigma_{1}\left(P_{c}(\omega)+P_{c}\left(\omega_{0}\right)\right) f\right\rangle ; \\
\alpha^{\omega}= & -\frac{1}{2}\left\langle\sigma_{1} R, \sigma_{3} \partial_{\omega} R\right\rangle ; \\
\alpha^{f}= & \frac{1}{2} \sigma_{1} \sigma_{3} P_{c}\left(\omega_{0}\right)\left(P_{c}(\omega)-P_{c}\left(\omega_{0}\right)\right) f .
\end{aligned}
$$

Proof. Everything is straightforward except for (7.20), which we now prove. We will sum over repeated indexes. We substitute $U$ using (3.13) getting

$$
\begin{aligned}
& \beta=\frac{1}{2}\left\langle e^{-\mathrm{i} \sigma_{3} \vartheta} \sigma_{1} \sigma_{3} \Phi, \quad\right\rangle+\frac{1}{2}\left\langle e^{-\mathrm{i} \sigma_{3} \vartheta} \sigma_{1} \sigma_{3} P_{c}(\omega) f,\right\rangle+ \\
& \frac{1}{2}\left[z_{j}\left\langle e^{-\mathrm{i} \sigma_{3} \vartheta} \sigma_{1} \sigma_{3} \xi_{j}, \quad\right\rangle-\bar{z}_{j}\left\langle e^{-\mathrm{i} \sigma_{3} \vartheta} \sigma_{3} \xi_{j}, \quad\right\rangle\right] .
\end{aligned}
$$

When we decompose $\frac{1}{2} e^{-\mathrm{i} \sigma_{3} \vartheta} \sigma_{1} \sigma_{3} \Phi$ like $\nabla F$ in (4.5), we obtain

$$
\begin{aligned}
& \frac{1}{2}\left\langle e^{-\mathrm{i} \sigma_{3} \vartheta} \sigma_{1} \sigma_{3} \Phi, \quad\right\rangle=-\frac{q}{q^{\prime}}\left\langle e^{-\mathrm{i} \sigma_{3} \vartheta} \sigma_{3} \partial_{\omega} \Phi, \quad\right\rangle \\
& -\frac{1}{2}\left\langle\sigma_{3} \Phi, \xi_{j}\right\rangle\left(\left\langle e^{-\mathrm{i} \sigma_{3} \vartheta} \sigma_{3} \xi_{j}, \quad\right\rangle-\left\langle e^{-\mathrm{i} \sigma_{3} \vartheta} \sigma_{1} \sigma_{3} \xi_{j}, \quad\right\rangle\right) \\
& -\frac{1}{2}\left\langle e^{-\mathrm{i} \sigma_{3} \vartheta} P_{c}\left(\mathcal{H}_{\omega}^{*}\right) \sigma_{3} \Phi, \quad\right\rangle
\end{aligned}
$$


with by $(4.2)$

$$
-\frac{q}{q^{\prime}}\left\langle e^{-\mathrm{i} \sigma_{3} \vartheta} \sigma_{3} \partial_{\omega} \Phi, \quad\right\rangle=\frac{q}{q^{\prime}}\left\langle R, \sigma_{3} \partial_{\omega}^{2} \Phi\right\rangle d \omega-\mathrm{i} \frac{q}{q^{\prime}}\left(q^{\prime}+\left\langle R, \partial_{\omega} \Phi\right\rangle\right) d \vartheta .
$$

Substituting slightly manipulated versions of the formulas in Lemmas 4.1-4.2, in particular using $\sigma_{3} P_{c}(\omega)=P_{c}(\omega)^{*} \sigma_{3}, \sigma_{1} P_{c}(\omega)=P_{c}(\omega) \sigma_{1}$ and $\sigma_{1} \sigma_{3}=-\sigma_{1} \sigma_{3}$, and summing over repeated indexes, we get

$$
\begin{aligned}
& \beta_{0}=-\mathrm{i} q d \vartheta+\frac{1}{2} \bar{z}_{j}\left(\left\langle\sigma_{1} \sigma_{1} \xi_{j}, \sigma_{3} \partial_{\omega} R\right\rangle d \omega+\mathrm{i}\left\langle\xi_{j}, R\right\rangle d \vartheta-\left\langle e^{-\mathrm{i} \sigma_{3} \vartheta} \sigma_{3} \xi_{j}, \quad\right\rangle\right) \\
& +\frac{1}{2} z_{j}\left(\left\langle\sigma_{1} \xi_{j}, \sigma_{3} \partial_{\omega} R\right\rangle d \omega+\mathrm{i}\left\langle\xi_{j}, \sigma_{1} R\right\rangle d \vartheta+\left\langle e^{-\mathrm{i} \sigma_{3} \vartheta} \sigma_{1} \sigma_{3} \xi_{j}, \quad\right\rangle\right) \\
& +\frac{1}{2}\left\langle f, \sigma_{3} \sigma_{1}\left(1-P_{c}(\omega) P_{c}\left(\omega_{0}\right)\right) f^{\prime}\right\rangle+\frac{1}{2}\left\langle f, \sigma_{3} \sigma_{1} P_{c}(\omega) e^{-\mathrm{i} \sigma_{3} \vartheta}\right\rangle \\
& +\frac{1}{2}\left\langle\sigma_{1} P_{c}(\omega) f, \sigma_{3} \partial_{\omega} R\right\rangle d \omega+\frac{\mathrm{i}}{2}\left\langle P_{c}(\omega) f, \sigma_{1} R\right\rangle d \vartheta .
\end{aligned}
$$

Hence

$$
\begin{aligned}
& \beta_{0}=\mathrm{i}\left(-q+\frac{1}{2}\left\langle R, \sigma_{1} R\right\rangle\right) d \vartheta+\frac{1}{2}\left\langle\sigma_{1} R, \sigma_{3} \partial_{\omega} R\right\rangle d \omega+ \\
& +\frac{1}{2}\left\langle\sigma_{1} \sigma_{3}\left(1-P_{c}\left(\omega_{0}\right) P_{c}(\omega)\right) f, f^{\prime}\right\rangle+ \\
& +\frac{1}{2}\left(z_{j}\left\langle e^{-\mathrm{i} \sigma_{3} \vartheta} \sigma_{1} \sigma_{3} \xi_{j}, \quad\right\rangle-\bar{z}_{j}\left\langle e^{-\mathrm{i} \sigma_{3} \vartheta} \sigma_{3} \xi_{j}, \quad\right\rangle\right)+ \\
& +\frac{1}{2}\left\langle e^{-\mathrm{i} \sigma_{3} \vartheta} \sigma_{1} \sigma_{3} P_{c}(\omega) f, \quad\right\rangle .
\end{aligned}
$$

By (3.13) we have

$$
d \psi=\frac{1}{2}\left\langle\sigma_{3} \Phi, \partial_{\omega} R\right\rangle d \omega+\frac{1}{2}\left\langle\sigma_{3} \Phi, \xi_{j}\right\rangle\left(d z_{j}-d \bar{z}_{j}\right)+\frac{1}{2}\left\langle\sigma_{3} \Phi, P_{c}(\omega) f^{\prime} \quad\right\rangle .
$$

Applying to (7.25) Lemmas 4.1-4.2, the fact that, in particular, we have

$$
P_{c}(\omega) f^{\prime}(U)=P_{c}(\omega) P_{c}\left(\omega_{0}\right) f^{\prime}(U)=P_{c}(\omega)\left[-\partial_{\omega} R d \omega-\mathrm{i} \sigma_{3} R d \vartheta+e^{-\mathrm{i} \sigma_{3} \vartheta} \mathbb{1}\right],
$$

and the identities (7.27)-(7.28) below, we get $d \psi=$

$$
\begin{aligned}
& =\frac{1}{2}\left\langle\sigma_{3} \Phi, \xi_{j}\right\rangle\left(\left\langle e^{-\mathrm{i} \sigma_{3} \vartheta} \sigma_{3} \xi_{j}, \quad\right\rangle-\left\langle e^{-\mathrm{i} \sigma_{3} \vartheta} \sigma_{1} \sigma_{3} \xi_{j}, \quad\right\rangle\right) \\
& +\frac{1}{2}\left\langle e^{-\mathrm{i} \sigma_{3} \vartheta} P_{c}\left(\mathcal{H}_{\omega}^{*}\right) \sigma_{3} \Phi, \quad\right\rangle \\
& +\frac{q}{q^{\prime}}\left\langle\sigma_{3} \partial_{\omega} \Phi, \partial_{\omega} R\right\rangle d \omega-\frac{\mathrm{i}}{2}\left\langle\sigma_{3} \Phi, P_{N_{g}^{\perp}\left(\mathcal{H}_{\omega}^{*}\right)} \sigma_{3} R\right\rangle d \vartheta .
\end{aligned}
$$

To get the last line of (7.26) we have used: 


$$
\begin{gathered}
\frac{1}{2}\left\langle\sigma_{3} \Phi, \partial_{\omega} R\right\rangle-\frac{1}{2}\left\langle\sigma_{3} \Phi, \xi_{j}\right\rangle\left\langle\sigma_{3} \xi_{j}, \partial_{\omega} R\right\rangle \\
-\frac{1}{2}\left\langle\sigma_{3} \Phi, \sigma_{1} \xi_{j}\right\rangle\left\langle\sigma_{1} \sigma_{3} \xi_{j}, \partial_{\omega} R\right\rangle-\frac{1}{2}\left\langle\sigma_{3} \Phi, P_{c}(\omega) \partial_{\omega} R\right\rangle=\frac{1}{2}\left\langle\sigma_{3} \Phi, \partial_{\omega} R\right\rangle \\
-\frac{1}{2}\left[\left\langle\sigma_{3} \Phi, \partial_{\omega} R\right\rangle-\frac{1}{q^{\prime}}\left\langle\sigma_{3} \Phi, \sigma_{3} \Phi\right\rangle\left\langle\sigma_{3} \partial_{\omega} \Phi, \partial_{\omega} R\right\rangle\right]=\frac{2 q}{2 q^{\prime}}\left\langle\sigma_{3} \partial_{\omega} \Phi, \partial_{\omega} R\right\rangle ; \\
-\frac{\mathrm{i}}{2}\left\langle\sigma_{3} \Phi, \xi_{j}\right\rangle\left\langle\sigma_{3} \xi_{j}, \sigma_{3} R\right\rangle-\frac{\mathrm{i}}{2}\left\langle\sigma_{3} \Phi, \sigma_{1} \xi_{j}\right\rangle\left\langle\sigma_{1} \sigma_{3} \xi_{j}, \sigma_{3} R\right\rangle \\
-\frac{\mathrm{i}}{2}\left\langle\sigma_{3} \Phi, P_{c}(\omega) \sigma_{3} R\right\rangle=-\frac{\mathrm{i}}{2}\left\langle\sigma_{3} \Phi, P_{N_{g}^{\perp}\left(\mathcal{H}_{\omega}^{*}\right)} \sigma_{3} R\right\rangle .
\end{gathered}
$$

Let us consider the sum (7.19). There are various cancelations. The first and second (resp. the first term of the third) line of (7.26) cancel with the second and third lines of (7.22) (resp. the first term of the rhs of (7.23)). The last three terms in $\operatorname{rhs}(7.21)$ cancel with the last two lines of (7.24). The $-\mathrm{i} q d \vartheta$ term in the rhs of (7.24) cancels with the $-\mathrm{i} q d \vartheta$ term in (7.23). Adding the second term of the third line of (7.26) with the last term in the rhs of (7.23) we get the product of $i$ times the following quantities:

$$
\begin{aligned}
& -\frac{1}{2}\left\langle\sigma_{3} \Phi, P_{N_{g}^{\perp}\left(\mathcal{H}_{\omega}^{*}\right)} \sigma_{3} R\right\rangle-\frac{q}{q^{\prime}}\left\langle R, \partial_{\omega} \Phi\right\rangle=-\frac{1}{2}\langle\Phi, R\rangle \\
& +\frac{1}{2}\left\langle\sigma_{3} \Phi, P_{N_{g}\left(\mathcal{H}_{\omega}\right)} \sigma_{3} R\right\rangle-\frac{q}{q^{\prime}}\left\langle R, \partial_{\omega} \Phi\right\rangle \\
& =-\frac{1}{2}\langle\Phi, R\rangle+\frac{1}{2 q^{\prime}}\left\langle\sigma_{3} R, \Phi\right\rangle\left\langle\sigma_{3} \Phi, \partial_{\omega} \Phi\right\rangle \\
& +\frac{1}{2 q^{\prime}}\left\langle\sigma_{3} R, \sigma_{3} \partial_{\omega} \Phi\right\rangle\left\langle\sigma_{3} \Phi, \sigma_{3} \Phi\right\rangle-\frac{q}{q^{\prime}}\left\langle R, \partial_{\omega} \Phi\right\rangle=0,
\end{aligned}
$$

where for the second equality we have used

$$
P_{N_{g}\left(\mathcal{H}_{\omega}\right)}=\frac{1}{q^{\prime}} \sigma_{3} \Phi\left\langle\sigma_{3} \partial_{\omega} \Phi, \quad\right\rangle+\frac{1}{q^{\prime}} \partial_{\omega} \Phi\langle\Phi, \quad\rangle .
$$

The last equality in (7.29) can be seen as follows. The two terms in the third line in (7.29) are both equal to 0. Indeed, $\left\langle\sigma_{3} \Phi, \partial_{\omega} \Phi\right\rangle=0$ and, by $R \in N_{g}^{\perp}\left(\mathcal{H}_{\omega}^{*}\right)$ and $\Phi \in N_{g}\left(\mathcal{H}_{\omega}^{*}\right),\langle R, \Phi\rangle=0$. The two terms in the fourth line in (7.29) cancel each other. Then we get formulas for $\alpha^{\omega}$ and $\alpha^{f}$. We get $\alpha^{\vartheta}$ also by

$$
\left\|P_{c}(\omega) f\right\|_{2}^{2}=\|f\|_{2}^{2}+\left\langle\left(P_{c}(\omega)-P_{c}\left(\omega_{0}\right)\right) f, \sigma_{1}\left(P_{c}(\omega)+P_{c}\left(\omega_{0}\right)\right) f\right\rangle .
$$

We have, summing over repeated indexes (also on $j$ and $\bar{j}$ ):

Lemma 7.3. We have

$$
i_{Y} \Omega_{0}=\mathrm{i} q^{\prime} Y_{\vartheta} d \omega-\mathrm{i} q^{\prime} Y_{\omega} d \vartheta+\left(Y_{j} d \bar{z}_{j}-Y_{\bar{j}} d z_{j}\right)+\left\langle\sigma_{1} \sigma_{3} Y_{f}, f^{\prime} \quad\right\rangle .
$$


For $a_{1}$ given by (7.15), and for $\Gamma=i_{Y} \widetilde{\Omega}$, we have

$$
\begin{aligned}
\Gamma_{\omega}= & a_{1} Y_{\vartheta}+\left\langle\sigma_{1} \sigma_{3} \xi_{j}, \partial_{\omega} R\right\rangle Y_{j}-\left\langle\sigma_{3} \xi_{j}, \partial_{\omega} R\right\rangle Y_{\bar{j}} \\
& +\left\langle Y_{f}, \sigma_{3} \sigma_{1} P_{c}(\omega) \partial_{\omega} R\right\rangle ; \\
-\Gamma_{\vartheta}= & a_{1} Y_{\omega}-\mathrm{i}\left\langle\sigma_{1} \sigma_{3} \xi_{j}, \sigma_{3} R\right\rangle Y_{j}+\mathrm{i}\left\langle\sigma_{3} \xi_{j}, \sigma_{3} R\right\rangle Y_{\bar{j}} \\
& -\mathrm{i}\left\langle Y_{f}, \sigma_{3} \sigma_{1} P_{c}(\omega) \sigma_{3} R\right\rangle ; \\
-\Gamma_{j}= & \left\langle\sigma_{1} \sigma_{3} \xi_{j}, \partial_{\omega} R\right\rangle Y_{\omega}+\mathrm{i}\left\langle\sigma_{1} \sigma_{3} \xi_{j}, \sigma_{3} R\right\rangle Y_{\vartheta} ; \\
\Gamma_{\bar{j}}= & \left\langle\sigma_{3} \xi_{j}, \partial_{\omega} R\right\rangle Y_{\omega}+\mathrm{i}\left\langle\sigma_{3} \xi_{j}, \sigma_{3} R\right\rangle Y_{\vartheta} ; \\
\sigma_{3} \sigma_{1} \Gamma_{f}= & \left(P_{c}\left(\omega_{0}\right) P_{c}(\omega)-1\right) Y_{f} \\
& +Y_{\omega} P_{c}\left(\omega_{0}\right) P_{c}(\omega) \partial_{\omega} R+\mathrm{i} Y_{\vartheta} P_{c}\left(\omega_{0}\right) P_{c}(\omega) \sigma_{3} R .
\end{aligned}
$$

In particular, for $\gamma=i_{Y^{t}} \Omega_{t}=i_{Y^{t}} \Omega_{0}+t i_{Y^{t}} \widetilde{\Omega}$ we have

$$
\begin{aligned}
\gamma_{\omega}= & \left(\mathrm{i} q^{\prime}+t a_{1}\right)\left(Y^{t}\right)_{\vartheta}+t\left\langle\sigma_{1} \sigma_{3} \xi_{j}, \partial_{\omega} R\right\rangle\left(Y^{t}\right)_{j}-t\left\langle\sigma_{3} \xi_{j}, \partial_{\omega} R\right\rangle\left(Y^{t}\right)_{\bar{j}} \\
& +t\left\langle\left(Y^{t}\right)_{f}, \sigma_{3} \sigma_{1} P_{c}\left(\omega_{0}\right) P_{c}(\omega) \partial_{\omega} R\right\rangle ; \\
-\gamma_{\vartheta}= & \left(\mathrm{i} q^{\prime}+t a_{1}\right)\left(Y^{t}\right)_{\omega}-\mathrm{i} t\left\langle\sigma_{1} \sigma_{3} \xi_{j}, \sigma_{3} R\right\rangle\left(Y^{t}\right)_{j}+\mathrm{i} t\left\langle\sigma_{3} \xi_{j}, \sigma_{3} R\right\rangle\left(Y^{t}\right)_{\bar{j}} \\
& -\mathrm{i} t\left\langle\left(Y^{t}\right)_{f}, \sigma_{3} \sigma_{1} P_{c}\left(\omega_{0}\right) P_{c}(\omega) \sigma_{3} R\right\rangle ; \\
-\gamma_{j}= & \left(Y^{t}\right)_{\bar{j}}+t\left\langle\sigma_{1} \sigma_{3} \xi_{j}, \partial_{\omega} R\right\rangle\left(Y^{t}\right)_{\omega}+\mathrm{i} t\left\langle\sigma_{1} \sigma_{3} \xi_{j}, \sigma_{3} R\right\rangle\left(Y^{t}\right)_{\vartheta} ; \\
\gamma_{\bar{j}}= & \left(Y^{t}\right)_{j}+t\left\langle\sigma_{3} \xi_{j}, \partial_{\omega} R\right\rangle\left(Y^{t}\right)_{\omega}+\mathrm{i} t\left\langle\sigma_{3} \xi_{j}, \sigma_{3} R\right\rangle\left(Y^{t}\right)_{\vartheta} ; \\
\sigma_{3} \sigma_{1} \gamma_{f}= & \left(Y^{t}\right)_{f}+t\left(P_{c}\left(\omega_{0}\right) P_{c}(\omega)-1\right)\left(Y^{t}\right)_{f}+ \\
& +t\left(Y^{t}\right)_{\omega} P_{c}\left(\omega_{0}\right) P_{c}(\omega) \partial_{\omega} R+t \mathrm{i}\left(Y^{t}\right)_{\vartheta} P_{c}\left(\omega_{0}\right) P_{c}(\omega) \sigma_{3} R .
\end{aligned}
$$

Proof. (7.30) is trivial. (7.32) follows immediately from (7.30)-(7.31). In the following formulas we denote $P_{c}=P_{c}(\omega), P_{c}^{0}=P_{c}\left(\omega_{0}\right)$ and we sum on repeated indexes. We can split $\widetilde{\Omega}=\widehat{\Omega}+\widehat{\Omega}_{1}$ with, see (7.13),

$$
\begin{aligned}
& \widehat{\Omega}_{1}=\left\langle\left(P_{c}^{0} P_{c}-1\right) f^{\prime}, \sigma_{3} \sigma_{1} f^{\prime}\right\rangle, \\
& \widehat{\Omega}=a_{1} d \vartheta \wedge d \omega+d z_{j} \wedge\left(\left\langle\sigma_{1} \sigma_{3} \xi_{j}, \partial_{\omega} R\right\rangle d \omega+\mathrm{i}\left\langle\sigma_{1} \sigma_{3} \xi_{j}, \sigma_{3} R\right\rangle d \vartheta\right) \\
& -d \bar{z}_{j} \wedge\left(\left\langle\sigma_{3} \xi_{j}, \partial_{\omega} R\right\rangle d \omega+\mathrm{i}\left\langle\sigma_{3} \xi_{j}, \sigma_{3} R\right\rangle d \vartheta\right)+ \\
& \left\langle P_{c} P_{c}^{0} f^{\prime}, \sigma_{3} \sigma_{1} P_{c} \partial_{\omega} R\right\rangle \wedge d \omega+\mathrm{i}\left\langle P_{c} P_{c}^{0} f^{\prime}, \sigma_{3} \sigma_{1} P_{c} \sigma_{3} R\right\rangle \wedge d \vartheta .
\end{aligned}
$$

Then

$$
i_{Y} \widehat{\Omega}_{1}=\left\langle\sigma_{1} \sigma_{3}\left(P_{c}^{0} P_{c}-1\right) Y_{f}, f^{\prime}\right\rangle
$$

and

$$
\begin{aligned}
& i_{Y} \widehat{\Omega}=\left[a_{1} Y_{\vartheta}+Y_{j}\left\langle\sigma_{1} \sigma_{3} \xi_{j}, \partial_{\omega} R\right\rangle-Y_{\bar{j}}\left\langle\sigma_{3} \xi_{j}, \partial_{\omega} R\right\rangle+\left\langle Y_{f}, \sigma_{3} \sigma_{1} P_{c} \partial_{\omega} R\right\rangle\right] d \omega+ \\
& {\left[-a_{1} Y_{\omega}+\mathrm{i} Y_{j}\left\langle\sigma_{1} \sigma_{3} \xi_{j}, \sigma_{3} R\right\rangle-\mathrm{i} Y_{\bar{j}}\left\langle\sigma_{3} \xi_{j}, \sigma_{3} R\right\rangle+\mathrm{i}\left\langle Y_{f}, \sigma_{3} \sigma_{1} P_{c} \sigma_{3} R\right\rangle\right] d \vartheta} \\
& -\left(\left\langle\sigma_{1} \sigma_{3} \xi_{j}, \partial_{\omega} R\right\rangle Y_{\omega}+\mathrm{i}\left\langle\sigma_{1} \sigma_{3} \xi_{j}, \sigma_{3} R\right\rangle Y_{\vartheta}\right) d z_{j} \\
& +\left(\left\langle\sigma_{3} \xi_{j}, \partial_{\omega} R\right\rangle Y_{\omega}+\mathrm{i}\left\langle\sigma_{3} \xi_{j}, \sigma_{3} R\right\rangle Y_{\vartheta}\right) d \bar{z}_{j} \\
& -\left\langle f^{\prime}, Y_{\omega} \sigma_{3} \sigma_{1} P_{c}^{0} P_{c} \partial_{\omega} R+\mathrm{i} Y_{\vartheta} \sigma_{3} \sigma_{1} P_{c}^{0} P_{c} \sigma_{3} R\right\rangle .
\end{aligned}
$$


Remark 7.4. If we choose $\gamma=-\mathrm{i} \alpha$ in Lemma 7.3 with the $\alpha$ of (7.19), and if $\mathcal{F}_{t}$ is the flow of $Y^{t}$, then $\left(Y^{t}\right)_{\vartheta} \neq 0$ is an obstruction to the fact that, for $0<t \leq 1, K \circ \mathcal{F}_{t}$ is the hamiltonian of the sort of semilinear NLS that (6.1) is. Indeed $\left(Y^{t}\right)_{f}=-t \mathrm{i}\left(Y^{t}\right)_{\vartheta} P_{c}\left(\omega_{0}\right) P_{c}(\omega) \sigma_{3} R+\mathcal{S}\left(\mathbb{R}^{3}, \mathbb{C}^{2}\right)$. Then if we substitute $f$ with $f-\mathrm{i}\left(Y^{1}\right)_{\vartheta} P_{c}\left(\omega_{0}\right) P_{c}(\omega) \sigma_{3} R+\ldots$ in $\left\langle\mathcal{H}_{\omega} f, \sigma_{3} \sigma_{1} f\right\rangle$ we obtain a term of the form $\left(Y^{1}\right)_{\vartheta}^{2}\left\langle\mathcal{H}_{\omega} f, \sigma_{3} \sigma_{1} f\right\rangle$. To avoid terms like this, we want flows defined from fields with $\left(Y^{t}\right)_{\vartheta}=0$. To this effect we add a correction to $\alpha$.

We first consider the hamiltonian fields of $\vartheta$ and $\omega$.

Lemma 7.5. Consider the vectorfield $X_{\vartheta}^{t}\left(\right.$ resp. $\left.X_{\omega}^{t}\right)$ defined by $i_{X_{\vartheta}^{t}} \Omega_{t}=-\mathrm{i} d \vartheta$ (resp. $\left.i_{X_{\omega}^{t}} \Omega_{t}=-\mathrm{i} d \omega\right)$. Then we have $\left(\right.$ here $P_{c}=P_{c}\left(\mathcal{H}_{\omega}\right)$ and $P_{c}^{0}=P_{c}\left(\mathcal{H}_{\omega_{0}}\right)$ ):

$$
\begin{aligned}
& X_{\vartheta}^{t}=\left(X_{\vartheta}^{t}\right)_{\omega}\left[\frac{\partial}{\partial \omega}-t\left\langle\sigma_{3} \xi_{j}, \partial_{\omega} R\right\rangle \frac{\partial}{\partial z_{j}}-t\left\langle\sigma_{1} \sigma_{3} \xi_{j}, \partial_{\omega} R\right\rangle \frac{\partial}{\partial \bar{z}_{j}}\right. \\
& \left.-t P_{c}^{0}\left(1+t P_{c}-t P_{c}^{0}\right)^{-1} P_{c}^{0} P_{c} \partial_{\omega} R\right], \\
& X_{\omega}^{t}=\left(X_{\omega}^{t}\right)_{\vartheta}\left[\frac{\partial}{\partial \vartheta}-\mathrm{i} t\left\langle\xi_{j}, R\right\rangle \frac{\partial}{\partial z_{j}}+\mathrm{i} t\left\langle\sigma_{1} \xi_{j}, R\right\rangle \frac{\partial}{\partial \bar{z}_{j}}\right. \\
& \left.-\mathrm{i} t P_{c}^{0}\left(1+t P_{c}-t P_{c}^{0}\right)^{-1} P_{c}^{0} P_{c} \sigma_{3} R\right],
\end{aligned}
$$

where, for the $a_{1}$ of (7.15), we have

$$
\begin{gathered}
\left(X_{\vartheta}^{t}\right)_{\omega}=\frac{\mathrm{i}}{\mathrm{i} q^{\prime}+t a_{1}+t a_{2}}=-\left(X_{\omega}^{t}\right)_{\vartheta} \\
a_{2}:=\mathrm{i} t\left\langle\sigma_{3} \xi_{j}, \partial_{\omega} R\right\rangle\left\langle\sigma_{1} \xi_{j}, R\right\rangle-\mathrm{i} t\left\langle\sigma_{1} \sigma_{3} \xi_{j}, \partial_{\omega} R\right\rangle\left\langle\xi_{j}, R\right\rangle+ \\
+\mathrm{i} t\left\langle P_{c}^{0}\left(1+t P_{c}-t P_{c}^{0}\right)^{-1} P_{c}^{0} P_{c} \partial_{\omega} R, \sigma_{3} \sigma_{1} P_{c} \sigma_{3} R\right\rangle .
\end{gathered}
$$

Proof. By (7.32) for $\gamma=-\mathrm{i} d \vartheta, X_{\vartheta}^{t}$ satisfies

$$
\begin{aligned}
& \left(X_{\vartheta}^{t}\right)_{\vartheta}=0 ; \\
& \mathrm{i}=\left(\mathrm{i} q^{\prime}+t a_{1}\right)\left(X_{\vartheta}^{t}\right)_{\omega}-\mathrm{i} t\left\langle\sigma_{1} \sigma_{3} \xi_{j}, \sigma_{3} R\right\rangle\left(X_{\vartheta}^{t}\right)_{j}+ \\
& +\mathrm{i} t\left\langle\sigma_{3} \xi_{j}, \sigma_{3} R\right\rangle\left(X_{\vartheta}^{t}\right)_{\bar{j}}-\mathrm{i} t\left\langle\left(X_{\vartheta}^{t}\right)_{f}, \sigma_{3} \sigma_{1} P_{c} \sigma_{3} R\right\rangle ; \\
& \left(X_{\vartheta}^{t}\right)_{f}=t\left(1-P_{c}^{0} P_{c}\right)\left(X_{\vartheta}^{t}\right)_{f}-t\left(X_{\vartheta}^{t}\right)_{\omega} P_{c}^{0} P_{c} \partial_{\omega} R ; \\
& \left(X_{\vartheta}^{t}\right)_{\bar{j}}=-t\left(X_{\vartheta}^{t}\right)_{\omega}\left\langle\sigma_{1} \sigma_{3} \xi_{j}, \partial_{\omega} R\right\rangle ;\left(X_{\vartheta}^{t}\right)_{j}=-t\left(X_{\vartheta}^{t}\right)_{\omega}\left\langle\sigma_{3} \xi_{j}, \partial_{\omega} R\right\rangle .
\end{aligned}
$$

This yields (7.33) for $X_{\vartheta}^{t}$ and the first equality in (7.34). By (7.32) for $\gamma=-\mathrm{i} d \omega$, $X_{\omega}^{t}$ satisfies

$$
\begin{aligned}
& \left(X_{\omega}^{t}\right)_{\omega}=0 \\
& -\mathrm{i}-\mathrm{i} q^{\prime}\left(X_{\omega}^{t}\right)_{\vartheta}=t a_{1}\left(X_{\omega}^{t}\right)_{\vartheta}+t\left\langle\sigma_{1} \sigma_{3} \xi_{j}, \partial_{\omega} R\right\rangle\left(X_{\omega}^{t}\right)_{j}- \\
& -t\left\langle\sigma_{1} \sigma_{3} \xi_{j}, \partial_{\omega} R\right\rangle\left(X_{\omega}^{t}\right)_{\bar{j}}+t\left\langle\left(X_{\omega}^{t}\right)_{f}, \sigma_{3} \sigma_{1} P_{c} \partial_{\omega} R\right\rangle ; \\
& \left(X_{\omega}^{t}\right)_{f}=t\left(1-P_{c}^{0} P_{c}\right)\left(X_{\omega}^{t}\right)_{f}-\mathrm{i} t\left(X_{\omega}^{t}\right)_{\vartheta} P_{c}^{0} P_{c} \sigma_{3} R ; \\
& \left(X_{\omega}^{t}\right)_{\bar{j}}=-\mathrm{i} t\left(X_{\omega}^{t}\right)_{\vartheta}\left\langle\sigma_{1} \sigma_{3} \xi_{j}, \sigma_{3} R\right\rangle ; \quad\left(X_{\omega}^{t}\right)_{j}=-\mathrm{i} t\left(X_{\omega}^{t}\right)_{\vartheta}\left\langle\sigma_{3} \xi_{j}, \sigma_{3} R\right\rangle .
\end{aligned}
$$


This yields the rest of (7.33)-(7.34).

The following lemma is an immediate consequence of the formulas in Lemma 7.5 and of (7.16).

Lemma 7.6. For any $\left(K^{\prime}, S^{\prime}, K, S\right)$ we have

$$
\begin{aligned}
& \left|1-\left(X_{\vartheta}^{t}\right)_{\omega} q^{\prime}\right| \lesssim\|R\|_{H^{-K^{\prime},-S^{\prime}}}^{2} \\
& \left|\left(X_{\vartheta}^{t}\right)_{j}\right|+\left|\left(X_{\vartheta}^{t}\right)_{\bar{j}}\right|+\left\|\left(X_{\vartheta}^{t}\right)_{f}\right\|_{H^{K, S}} \lesssim\|R\|_{H^{-K^{\prime},-S^{\prime}}} .
\end{aligned}
$$

and

$$
\begin{aligned}
& \left|1+\left(X_{\omega}^{t}\right)_{\vartheta} q^{\prime}\right| \lesssim\|R\|_{H^{-K^{\prime},-S^{\prime}}}^{2}, \\
& \left|\left(X_{\omega}^{t}\right)_{j}\right|+\left|\left(X_{\omega}^{t}\right)_{j}\right|+\left\|\left(X_{\omega}^{t}\right)_{f}\right\|_{H^{-K^{\prime},-S^{\prime}}} \lesssim\|R\|_{H^{-K^{\prime},-S^{\prime}}} .
\end{aligned}
$$

Set $H_{c}^{K, S}(\omega)=P_{c}(\omega) H^{K, S}$ and denote

$$
\widetilde{\mathcal{P}}^{K, S}=\mathbb{C}^{m} \times H_{c}^{K, S}\left(\omega_{0}\right), \quad \mathcal{P}^{K, S}=\mathbb{R}^{2} \times \widetilde{\mathcal{P}}^{K, S}
$$

with elements $(\vartheta, \omega, z, f) \in \mathcal{P}^{K, S}$ and $(z, f) \in \widetilde{\mathcal{P}}^{K, S}$.

Lemma 7.7. We consider $\forall t \in[0,1]$ the hamiltonian field $X_{\vartheta}^{t}$ and the flow

$$
\frac{d}{d s} \Phi_{s}(t, U)=X_{\vartheta}^{t}\left(\Phi_{s}(t, U)\right), \Phi_{0}(t, U)=U .
$$

(1) For any $\left(K^{\prime}, S^{\prime}\right)$ there is a $s_{0}>0$ and a neighborhood $\mathcal{U}$ of $\mathbb{R} \times\left\{\left(\omega_{0}, 0,0\right)\right\}$ in $\mathcal{P}^{-K^{\prime},-S^{\prime}}$ such that the map $(s, t, U) \rightarrow \Phi_{s}(t, U)$ is smooth

$$
\left(-s_{0}, s_{0}\right) \times[0,1] \times\left(\mathcal{U} \cap\left\{\omega=\omega_{0}\right\}\right) \rightarrow \mathcal{P}^{-K^{\prime},-S^{\prime}} .
$$

(2) $\mathcal{U}$ can be chosen so that for any $t \in[0,1]$ there is another neighborhood $\mathcal{V}_{t}$ of $\mathbb{R} \times\left\{\left(\omega_{0}, 0,0\right)\right\}$ in $\mathcal{P}^{-K^{\prime},-S^{\prime}}$ s.t. the above map establishes a diffeomorphism

$$
\left(-s_{0}, s_{0}\right) \times\left(\mathcal{U} \cap\left\{\omega=\omega_{0}\right\}\right) \rightarrow \mathcal{V}_{t}
$$

(3) $f\left(\Phi_{s}(t, U)\right)-f(U)=G(t, s, z, f)$ is a smooth map for all $(K, S)$

$$
\left(-s_{0}, s_{0}\right) \times[0,1] \times\left(\mathcal{U} \cap\left\{\omega=\omega_{0}\right\}\right) \rightarrow H^{K, S}
$$

with $\|G(t, s, z, f)\|_{H^{K, S}} \leq C|s|\left(|z|+\|f\|_{H^{-K^{\prime},-S^{\prime}}}\right)$.

Proof. Claims (1)-(2) follow by Lemma 7.5 which implies $X_{\vartheta}^{t} \in C^{\infty}\left(\mathcal{U}, \mathcal{P}^{K, S}\right)$ for all $(K, S)$. Let $\zeta$ be any coordinate $z_{j}$ or $f$. Then, for $\zeta$ a scalar coordinate, we have

$$
\begin{aligned}
& \left|\zeta\left(\Phi_{s}(t, U)\right)-\zeta(U)\right| \leq \int_{-s}^{s}\left|\left(X_{\vartheta}^{t}\right)_{\zeta}\left(\Phi_{s^{\prime}}(t, U)\right)\right| d s^{\prime} \\
& \leq C|s| \sup _{\left|s^{\prime}\right| \leq s}\left(\left|z\left(\Phi_{s^{\prime}}(t, U)\right)\right|+\left\|f\left(\Phi_{s^{\prime}}(t, U)\right)\right\|_{H^{-K^{\prime},-S^{\prime}}}\right) .
\end{aligned}
$$


For $\zeta=f$ we have

$$
\left\|f\left(\Phi_{s}(t, U)\right)-f(U)\right\|_{H^{K, S}} \leq \int_{-s}^{s}\left\|\left(X_{\vartheta}^{t}\right)_{f}\left(\Phi_{s^{\prime}}(t, U)\right)\right\|_{H^{K, S}} d s^{\prime} \leq \operatorname{rhs}(7.44) .
$$

The above two formulas imply the following, which yields claim (3),

$$
\begin{aligned}
& \left\|f\left(\Phi_{s}(t, U)\right)-f(U)\right\|_{H^{K, S}} \leq C|s|\left(|z|+\|f\|_{H^{-K^{\prime},-S^{\prime}}}\right), \\
& \left|z\left(\Phi_{s}(t, U)\right)-z(U)\right| \leq C|s|\left(|z|+\|f\|_{H^{-K^{\prime},-S^{\prime}}}\right) .
\end{aligned}
$$

Lemma 7.8. We consider a scalar function $F(t, U)$ defined as follows:

$$
F\left(t, \Phi_{s}(t, U)\right)=\mathrm{i} \int_{0}^{s} \alpha_{\Phi_{s^{\prime}}(t, U)}\left(X_{\vartheta}^{t}\left(\Phi_{s^{\prime}}(t, U)\right)\right) d s^{\prime}, \text { where } \omega(U)=\omega_{0} .
$$

We have $F \in C^{\infty}([0,1] \times \mathcal{U}, \mathbb{R})$ for a neighborhood $\mathcal{U}$ of $\mathbb{R} \times\left\{\left(\omega_{0}, 0,0\right)\right\}$ in $\mathcal{P}^{-K^{\prime},-S^{\prime}}$. We have

$$
|F(t, U)| \leq C\left(K^{\prime}, S^{\prime}\right)\left|\omega-\omega_{0}\right|\left(|z|+\|f\|_{H^{-K^{\prime},-S^{\prime}}}\right)^{2} .
$$

We have (exterior differentiation only in $U$ )

$$
(\alpha+\mathrm{i} d F)\left(X_{\vartheta}^{t}\right)=0 .
$$

Proof. $F$ is smooth by (7.20) and Lemma 7.7. (7.48) follows by (7.41) and by

$$
\alpha_{U}\left(X_{\vartheta}^{t}(U)\right)+\mathrm{i} \frac{d}{d s}{ }_{\mid s=0} F\left(t, \Phi_{s}(t, U)\right)=0 .
$$

By (7.20) and (7.38) we have

$$
\left|\alpha\left(X_{\vartheta}^{t}\right)\right| \leq\left|\alpha^{\omega}\right|\left|\left(X_{\vartheta}^{t}\right)_{\omega}\right|+\left|\left\langle\alpha^{f},\left(X_{\vartheta}^{t}\right)_{f}\right\rangle\right| \lesssim\left(|z|+\|f\|_{H^{-K^{\prime},-S^{\prime}}}\right)^{2} .
$$

Then (7.47) follows by $|s| \approx\left|\omega\left(\Phi_{s}(t, U)\right)-\omega_{0}\right|$.

Lemma 7.9. Denote by $\mathcal{X}^{t}$ the vector field which solves

$$
i_{\mathcal{X}^{t}} \Omega_{t}=-\alpha-\mathrm{i} d F(t) .
$$

Then the following properties hold.

(1) There is a neighborhood $\mathcal{U}$ of $\mathbb{R} \times\left\{\left(\omega_{0}, 0,0\right)\right\}$ in $\mathcal{P}^{1,0}$ such that $\mathcal{X}^{t}(U) \in$ $C^{\infty}\left([0,1] \times \mathcal{U}, \mathcal{P}^{1,0}\right)$.

(2) We have $\left(\mathcal{X}^{t}\right)_{\vartheta} \equiv 0$.

(3) For constants $C\left(K, S, K^{\prime}, S^{\prime}\right)$ we have

$$
\begin{aligned}
& \left|\left(\mathcal{X}^{t}\right)_{\omega}+\frac{\|f\|_{2}^{2}}{2 q^{\prime}(\omega)}\right| \lesssim\left(|z|+\|f\|_{H^{-K^{\prime},-S^{\prime}}}\right)^{2} \\
& \left|\left(\mathcal{X}^{t}\right)_{j}\right|+\left|\left(\mathcal{X}^{t}\right)_{\bar{j}}\right|+\left\|\left(\mathcal{X}^{t}\right)_{f}\right\|_{H^{K, S}} \lesssim\left(|z|+\|f\|_{H^{-K^{\prime},-S^{\prime}}}\right) \times \\
& \times\left(\left|\omega-\omega_{0}\right|+|z|+\|f\|_{H^{-K^{\prime},-S^{\prime}}}+\|f\|_{L^{2}}^{2}\right) .
\end{aligned}
$$


(4) We have

$$
L_{\mathcal{X}^{t}} \frac{\partial}{\partial \vartheta}:=\left[\mathcal{X}^{t}, \frac{\partial}{\partial \vartheta}\right]=0
$$

Proof. Claim (1) follows from the regularity properties of $\alpha, F$ and $\Omega_{t}$ and from equations (7.54) and (7.56) below. (7.48) implies (2) by

$$
\mathrm{i}\left(\mathcal{X}^{t}\right)_{\vartheta}=\mathrm{i} d \vartheta\left(\mathcal{X}^{t}\right)=-i_{X_{\vartheta}^{t}} \Omega_{t}\left(\mathcal{X}^{t}\right)=i_{\mathcal{X}^{t}} \Omega_{t}\left(X_{\vartheta}^{t}\right)=-(\alpha+\mathrm{i} d F)\left(X_{\vartheta}^{t}\right)=0 .
$$

We have $\mathrm{i}\left(\mathcal{X}^{t}\right)_{\omega}=\mathrm{i} d \omega\left(\mathcal{X}^{t}\right)=-i_{X_{\omega}^{t}} \Omega_{t}\left(\mathcal{X}^{t}\right)$, so by (7.51) and (7.33) we get

$$
\begin{aligned}
& \mathrm{i}\left(\mathcal{X}^{t}\right)_{\omega}=i_{\mathcal{X}^{t}} \Omega_{t}\left(X_{\omega}^{t}\right)=-\left(X_{\omega}^{t}\right)_{\vartheta}\left[\alpha^{\vartheta}+t \partial_{j} F\left\langle\xi_{j}, R\right\rangle-t \partial_{j} F\left\langle\sigma_{1} \xi_{j}, R\right\rangle\right. \\
& \left.+t\left\langle\nabla_{f} F+\mathrm{i} \alpha^{f}, P_{c}^{0}\left(1+t P_{c}-t P_{c}^{0}\right)^{-1} P_{c}^{0} P_{c} \sigma_{3} R\right\rangle\right] .
\end{aligned}
$$

Then by $(7.20),(7.34),(7.15)$ and (7.35), we get the first inequality in (7.52):

$$
\left|\left(\mathcal{X}^{t}\right)_{\omega}+\frac{\|f\|_{2}^{2}}{2 q^{\prime}(\omega)}\right| \leq C\left(|z|+\|f\|_{H^{-K^{\prime},-S^{\prime}}}\right)^{2} .
$$

By (7.32) we have the following equations

$$
\begin{aligned}
\mathrm{i} \partial_{j} F & =\left(\mathcal{X}^{t}\right)_{\bar{j}}+t\left\langle\sigma_{1} \sigma_{3} \xi_{j}, \partial_{\omega} R\right\rangle\left(\mathcal{X}^{t}\right)_{\omega} \\
-\mathrm{i} \partial_{\bar{j}} F & =\left(\mathcal{X}^{t}\right)_{j}+t\left\langle\sigma_{3} \xi_{j}, \partial_{\omega} R\right\rangle\left(\mathcal{X}^{t}\right)_{\omega} \\
\sigma_{3} \sigma_{1}\left(\alpha^{f}+\mathrm{i} \nabla_{f} F\right) & =-\left(\mathcal{X}^{t}\right)_{f}-t\left(P_{c}^{0} P_{c}-1\right)\left(\mathcal{X}^{t}\right)_{f} \\
& -t\left(\mathcal{X}^{t}\right)_{\omega} P_{c}^{0} P_{c} \partial_{\omega} R .
\end{aligned}
$$

Formulas (7.56) imply

$$
\begin{aligned}
& \left|\left(\mathcal{X}_{\omega}^{t}\right)_{\bar{j}}\right| \leq\left|\partial_{j} F\right|+C\left(|z|+\|f\|_{H^{-K^{\prime},-S^{\prime}}}\right)\left|\left(\mathcal{X}^{t}\right)_{\omega}\right| \\
& \left|\left(\mathcal{X}_{\omega}^{t}\right)_{j}\right| \leq\left|\partial_{\bar{j}} F\right|+C\left(|z|+\|f\|_{H^{-K^{\prime},-S^{\prime}}}\right)\left|\left(\mathcal{X}^{t}\right)_{\omega}\right| \\
& \left\|\left(\mathcal{X}_{\omega}^{t}\right)_{f}\right\|_{H^{K, S}} \leq\left\|\alpha^{f}\right\|_{H^{K, S}}+\left\|\nabla_{f} F\right\|_{H^{K, S}}+C\left(|z|+\|f\|_{H^{-K^{\prime},-S^{\prime}}}\right)\left|\left(\mathcal{X}^{t}\right)_{\omega}\right|
\end{aligned}
$$

which with (7.55), (7.20) and Lemma (7.47) imply (7.52). (7.53) is a consequence of the following equalities, which we will justify below:

$$
0=L_{\frac{\partial}{\partial \vartheta}}\left(i_{\mathcal{X}^{t}} \Omega_{t}\right)=i_{\left[\frac{\partial}{\partial \vartheta}, \mathcal{X}^{t}\right]} \Omega_{t}+i_{\mathcal{X}^{t}} L_{\frac{\partial}{\partial \vartheta}} \Omega_{t}=i_{\left[\frac{\partial}{\partial \vartheta}, \mathcal{X}^{t}\right]} \Omega_{t} .
$$

The first equality is a consequence of $(7.51)$ and $L_{\frac{\partial}{\partial \vartheta}}(\alpha+\mathrm{i} d F)=0$. The latter is a consequence of $L_{\frac{\partial}{\partial \vartheta}} \alpha=0$ and $\frac{\partial}{\partial \vartheta} F=0$. Notice that $\frac{\partial}{\partial \vartheta} F=0$ can be proved observing that (7.48), (7.20) and Lemma 7.5 imply $X_{\vartheta}^{t} \frac{\partial}{\partial \vartheta} F=0$ and that on $\omega=\omega_{0}$ we have $\frac{\partial}{\partial \vartheta} F=0 . L_{\frac{\partial}{\partial \vartheta}} \alpha=0$ is a consequence of the Cartan "magic" formula $L_{X} \gamma=\left(i_{X} d+d i_{X}\right) \gamma$, of the definition (7.19) and of following equalities: 


$$
\begin{aligned}
& L_{\frac{\partial}{\partial \vartheta}} \beta=d i_{\frac{\partial}{\partial \vartheta}} \beta+i_{\frac{\partial}{\partial \vartheta}} d \beta=-\frac{\mathrm{i}}{2} d\left\langle\sigma_{1} U, U\right\rangle+\mathrm{i}\left\langle\sigma_{1} U, \quad\right\rangle=0 \\
& L_{\frac{\partial}{\partial \vartheta}} \beta_{0}=d i_{\frac{\partial}{\partial \vartheta}} \beta_{0}+i_{\frac{\partial}{\partial \vartheta}} d \beta_{0}=-\mathrm{i} d q-\mathrm{i} i_{\frac{\partial}{\partial \vartheta}}(d q \wedge d \vartheta)=-\mathrm{i} d q+\mathrm{i} d q \wedge i_{\frac{\partial}{\partial \vartheta}} d \vartheta=0 \\
& L_{\frac{\partial}{\partial \vartheta}} d \psi=d i_{\frac{\partial}{\partial \vartheta}} d \psi=\frac{1}{2} d \frac{\partial}{\partial \vartheta}\left\langle\sigma_{3} \Phi, R\right\rangle=0 .
\end{aligned}
$$

The second equality in (7.57) follows by the product rule for the Lie derivative. Finally, the third equality in (7.57) follows by $L_{\frac{\partial}{\partial \vartheta}} \Omega_{t}=(1-t) L_{\frac{\partial}{\partial \vartheta}} \Omega_{0}+t L_{\frac{\partial}{\partial \vartheta}} \Omega=$ 0 , consequence of $L_{\frac{\partial}{\partial \vartheta}} \Omega=0$ (resp. $L_{\frac{\partial}{\partial \vartheta}} \Omega_{0}=0$ ), in turn consequence of the first (resp. second) line in (7.58) and of the identity $L_{X} d \gamma=d L_{X} \gamma$.

We have:

Lemma 7.10. Consider the vectorfield $\mathcal{X}^{t}$ in Lemma 7.8 and denote by $\mathcal{F}_{t}(U)$ the corresponding flow. Then the flow $\mathcal{F}_{t}(U)$ for $U$ near $e^{\mathrm{i} \sigma_{3} \vartheta} \Phi_{\omega_{0}}$ is defined for all $t \in[0,1]$. We have $\vartheta \circ \mathcal{F}_{1}=\vartheta$. We have for $\ell=j, \bar{j}$,

$$
\begin{aligned}
& q\left(\omega\left(\mathcal{F}_{1}(U)\right)\right)=q(\omega(U))-\frac{\|f\|_{2}^{2}}{2}+\mathcal{E}_{\omega}(U) \\
& z_{\ell}\left(\mathcal{F}_{1}(U)\right)=z_{\ell}(U)+\mathcal{E}_{\ell}(U) \\
& f\left(\mathcal{F}_{1}(U)\right)=f(U)+\mathcal{E}_{f}(U)
\end{aligned}
$$

with

$$
\begin{gathered}
\left|\mathcal{E}_{\omega}(U)\right| \lesssim\left(\left|\omega-\omega_{0}\right|+|z|+\|f\|_{H^{-K^{\prime},-S^{\prime}}}\right)^{2} \\
\left|\mathcal{E}_{\ell}(U)\right|+\left\|\mathcal{E}_{f}(U)\right\|_{H^{K, S}} \lesssim\left(\left|\omega-\omega_{0}\right|+|z|+\|f\|_{H^{-K^{\prime},-S^{\prime}}}+\|f\|_{L^{2}}^{2}\right) \\
\times\left(\left|\omega-\omega_{0}\right|+|z|+\|f\|_{H^{-K^{\prime},-S^{\prime}}}\right) .
\end{gathered}
$$

For each $\zeta=\omega, z_{\ell}, f$ we have

$$
\mathcal{E}_{\zeta}(U)=\mathcal{E}_{\zeta}\left(\|f\|_{L^{2}}^{2}, \omega, z, f\right)
$$

with, for a neighborhood $\mathcal{U}^{-K^{\prime},-S^{\prime}}$ of $\mathbb{R} \times\left\{\left(\omega_{0}, 0,0\right)\right\}$ in $\mathcal{P}^{-K^{\prime},-S^{\prime}}$ and for some fixed $a_{0}>0$

$$
\mathcal{E}_{\zeta}(\varrho, \omega, z, f) \in C^{\infty}\left(\left(-a_{0}, a_{0}\right) \times \mathcal{U}^{-K^{\prime},-S^{\prime}}, \mathbb{C}\right)
$$

for $\zeta=\omega, z_{\ell}$ and with

$$
\mathcal{E}_{f}(\varrho, \omega, z, f) \in C^{\infty}\left(\left(-a_{0}, a_{0}\right) \times \mathcal{U}^{-K^{\prime},-S^{\prime}}, H^{K, S}\right) .
$$

Proof. We add a new variable $\varrho$. We define a new field by

$$
\begin{aligned}
& \mathrm{i}\left(Y^{t}\right)_{\omega}=-\left(X_{\omega}^{t}\right)_{\vartheta}\left[\alpha^{\vartheta}+\mathrm{i} \frac{\|f\|_{2}^{2}-\rho}{2}+t \partial_{j} F\left\langle\xi_{j}, R\right\rangle-t \partial_{j} F\left\langle\sigma_{1} \xi_{j}, R\right\rangle\right. \\
& \left.+t\left\langle\nabla_{f} F+\mathrm{i} \alpha^{f}, P_{c}^{0}\left(1+t P_{c}-t P_{c}^{0}\right)^{-1} P_{c}^{0} P_{c} \sigma_{3} R\right\rangle\right]
\end{aligned}
$$


by

$$
\begin{aligned}
\mathrm{i} \partial_{j} F & =\left(Y^{t}\right)_{\bar{j}}+t\left\langle\sigma_{1} \sigma_{3} \xi_{j}, \partial_{\omega} R\right\rangle\left(Y^{t}\right)_{\omega} \\
-\mathrm{i} \partial_{\bar{j}} F & =\left(Y^{t}\right)_{j}+t\left\langle\sigma_{3} \xi_{j}, \partial_{\omega} R\right\rangle\left(Y^{t}\right)_{\omega} \\
\sigma_{3} \sigma_{1}\left(\alpha^{f}+\mathrm{i} \nabla_{f} F\right) & =\left(Y^{t}\right)_{f}+t\left(P_{c}^{0} P_{c}-1\right)\left(Y^{t}\right)_{f} \\
& -t\left(Y^{t}\right)_{\omega} P_{c}^{0} P_{c} \partial_{\omega} R .
\end{aligned}
$$

and by $Y_{\rho}^{t}=2\left\langle\left(Y^{t}\right)_{f}, \sigma_{1} f\right\rangle$. Then $Y^{t}=Y^{t}(\omega, \rho, z, f)$ defines a new flow $\mathcal{G}_{t}(\rho, U)$, which reduces to $\mathcal{F}_{t}(U)$ in the invariant manifold defined by $\rho=\|f\|_{2}^{2}$. Notice that by $\rho(t)=\rho(0)+\int_{0}^{t} Y_{\rho}^{s} d s$ it is easy to conclude $\rho\left(\mathcal{G}_{1}(\rho, U)\right)=(U)+$ $O(\operatorname{rhs}(7.60))$. Using (7.39), (7.20) and (7.65) it is then easy to get

$$
q(\omega(t))=q(\omega(0))+\int_{0}^{t} q^{\prime}(\omega(s)) Y_{\omega}^{s} d s=q(\omega(0))-\int_{0}^{t} \frac{\rho(s)}{2} d s+O(\operatorname{rhs}(7.60)) .
$$

By standard arguments, see for example the proof of Lemma 4.3 [BC], we get

$$
\begin{aligned}
& q\left(\omega\left(\mathcal{G}_{1}(\rho, U)\right)\right)=q(\omega(U))-\frac{\rho}{2}+\mathcal{E}_{\omega}(\rho, U), \\
& z_{\ell}\left(\mathcal{G}_{1}(\rho, U)\right)=z_{\ell}(U)+\mathcal{E}_{\ell}(\rho, U), \\
& f\left(\mathcal{G}_{1}(\rho, U)\right)=f(U)+\mathcal{E}_{f}(\rho, U),
\end{aligned}
$$

with $\mathcal{E}_{\zeta}(\rho, U)$ satisfying (7.63) for $\zeta=\omega, z_{\ell}$ and (7.64) for $\zeta=f$. We have $\mathcal{E}_{\zeta}(U)=\mathcal{E}_{\zeta}\left(\|f\|_{2}, U\right)$ satisfying (7.60) for $\zeta=\omega$ and (7.61) for $\zeta=z_{\ell}, f$.

We have:

Lemma 7.11. Consider the flow $\mathcal{F}_{t}$ of Lemma 7.10. Then we have

$$
\mathcal{F}_{t}^{*} \Omega_{t}=\Omega_{0} .
$$

We have

$$
Q \circ \mathcal{F}_{1}=q .
$$

If $\chi$ is a function with $\partial_{\vartheta} \chi \equiv 0$, then $\partial_{\vartheta}\left(\chi \circ \mathcal{F}_{t}\right) \equiv 0$.

Proof. (7.68) is Darboux Theorem, see (7.3). Let $\mathcal{G}_{t}=\left(\mathcal{F}_{t}\right)^{-1}$. Then $\mathcal{G}_{t}^{*} \Omega_{0}=$ $\Omega_{t}$. We have $\mathcal{G}_{t}^{*} X_{q(\omega)}^{0}=X_{q(\omega) \circ \mathcal{G}_{t}}^{t}$ by

$$
i_{\mathcal{G}_{t}^{*} X_{q(\omega)}^{0}} \Omega_{t}=i_{\mathcal{G}_{t}^{*} X_{q}^{0}(\omega)} \mathcal{G}_{t}^{*} \Omega_{0}=\mathcal{G}_{t}^{*} i_{X_{q}^{0}(\omega)} \Omega_{0}=-\mathrm{i} d\left(q(\omega) \circ \mathcal{G}_{t}\right)=i_{X_{q(\omega)}^{t} \circ \mathcal{G}_{t}} \Omega_{t} .
$$

Then by $\left[\mathcal{X}^{t}, \frac{\partial}{\partial \vartheta}\right]=0$ for all $t$

$$
\frac{d}{d t} X_{q(\omega) \circ \mathcal{G}_{t}}^{t}=\frac{d}{d t} \mathcal{G}_{t}^{*} X_{q(\omega)}^{0}=-\frac{d}{d t} \mathcal{G}_{t}^{*} \frac{\partial}{\partial \vartheta}=-\mathcal{G}_{t}^{*}\left[\mathcal{X}^{1-t}, \frac{\partial}{\partial \vartheta}\right]=0 .
$$

So $X_{q(\omega) \circ \mathcal{G}_{1}}^{1}=X_{q(\omega)}^{0}$. Since by $(5.16)$ and (7.7) this implies $d\left(q \circ \mathcal{G}_{1}\right)=d Q$ and since there are points with $q \circ \mathcal{G}_{1}(U)=Q(U)$, we obtain (7.69). Finally, the last statement of Lemma 7.11 follows by (7.53) and by

$$
\frac{\partial}{\partial \vartheta} \mathcal{F}_{t}^{*} \chi=\left(\mathcal{F}_{t}^{*} \frac{\partial}{\partial \vartheta}\right)\left(\mathcal{F}_{t}^{*} \chi\right)=\mathcal{F}_{t}^{*}\left(\frac{\partial}{\partial \vartheta} \chi\right) .
$$




\section{Reformulation of (6.5) in the new coordinates}

We set

$$
H=K \circ \mathcal{F}_{1} .
$$

In the new coordinates $(6.5)$ becomes

$$
q^{\prime} \dot{\omega}=\frac{\partial H}{\partial \vartheta} \equiv 0, \quad q^{\prime} \dot{\vartheta}=-\frac{\partial H}{\partial \omega}
$$

and

$$
\begin{aligned}
& \mathrm{i} \dot{z}_{j}=\frac{\partial H}{\partial \bar{z}_{j}}, \quad \mathrm{i} \dot{\bar{z}}_{j}=-\frac{\partial H}{\partial z_{j}} \\
& \mathrm{i} \dot{f}=\sigma_{3} \sigma_{1} \nabla_{f} H .
\end{aligned}
$$

Recall that we are solving the initial value problem (1.1) and that we have chosen $\omega_{0}$ with $q\left(\omega_{0}\right)=\left\|u_{0}\right\|_{L_{x}^{2}}^{2}$. Correspondingly it is enough to focus on $(8.3)$ with $\omega=\omega_{0}$. For system (8.3) we prove :

Theorem 8.1. Then there exist $\varepsilon>0$ and $C>0$ such that for $|z(0)|+$ $\|f(0)\|_{H^{1}} \leq \epsilon<\varepsilon$ the corresponding solution of (8.3) is globally defined and there are $f_{ \pm} \in H^{1}$ with $\left\|f_{ \pm}\right\|_{H^{1}} \leq C \epsilon$ such that

$$
\lim _{t \rightarrow \pm \infty}\left\|e^{\mathrm{i} \vartheta(t) \sigma_{3}} f(t)-e^{\mathrm{i} t \sigma_{3} \Delta} f_{ \pm}\right\|_{H^{1}}=0
$$

where $\vartheta(t)$ is the variable associated to $U^{T}(t)=(u(t), \bar{u}(t))$ in (3.12) and (3.13). We also have

$$
\lim _{t \rightarrow \infty} z(t)=0
$$

In particular, it is possible to write $R(t, x)=A(t, x)+\tilde{f}(t, x)$ with $|A(t, x)| \leq$ $C_{N}(t)\langle x\rangle^{-N}$ for any $N$, with $\lim _{t \rightarrow \infty} C_{N}(t)=0$ and such that for any admissible pair $(r, p)$, i.e. (2.4), we have

$$
\|\widetilde{f}\|_{L_{t}^{r}\left(\mathbb{R}, W_{x}^{1, p}\right)} \leq C \epsilon .
$$

By Lemma 7.10, Theorem 8.1 implies Theorem 2.2. Indeed, if we denote $\left(\omega, z^{\prime}, f^{\prime}\right)$ the initial coordinates, and $\left(\omega_{0}, z, f\right)$ the coordinates in $(8.3)$, we have $z^{\prime}=z+O\left(|z|+\|f\|_{L_{x}^{2,-2}}\right)$ and $f^{\prime}=f+O\left(|z|+\|f\|_{L_{x}^{2,-2}}\right)$. The two error terms $O$ converge to 0 as $t \rightarrow \infty$. Hence the asymptotic behavior of $\left(z^{\prime}, f^{\prime}\right)$ and of $(z, f)$ is the same. We also have $q(\omega(t))=q\left(\omega_{0}\right)-\frac{\|f(t)\|_{2}^{2}}{2}+O\left(|z(t)|+\|f(t)\|_{L_{x}^{2,-2}}\right)$ which implies, say at $+\infty$

$$
\lim _{t \rightarrow+\infty} q(\omega(t))=\lim _{t \rightarrow+\infty}\left(q\left(\omega_{0}\right)-\frac{\left\|e^{\mathrm{i} t \sigma_{3} \Delta} f_{+}\right\|_{2}^{2}}{2}\right)=q\left(\omega_{0}\right)-\frac{\left\|f_{+}\right\|_{2}^{2}}{2}=q\left(\omega_{+}\right)
$$

for somewhere $\omega_{+}$is the unique element near $\omega_{0}$ for which the last inequality holds. So $\lim _{t \rightarrow+\infty} \omega(t)=\omega_{+}$.

In the rest of the paper we focus on Theorem 8.1. The main idea is that (8.3) is basically like the system considered in [BC]. Therefore Theorem 8.1 follows by the Birkhoff normal forms argument of $[\mathrm{BC}]$, supplemented with the various dispersive estimates in [CM]. 


\subsection{Taylor expansions}

Consider $U=e^{\mathrm{i} \sigma_{3} \vartheta}\left(\Phi_{\omega}+R\right)$ as in (3.12). Decompose $R$ as in (3.14). Set $u=\varphi+u_{c}$ with ${ }^{t}\left(P_{c}(\omega) f\right)=\left(u_{c}, \overline{u_{c}}\right)$. We have

$$
\begin{aligned}
& B\left(|u|^{2}\right)=B\left(\left|u_{c}\right|^{2}\right)+\int_{0}^{1}\left[\frac{\partial}{\partial u} B\left(|u|^{2}\right)_{\mid u=u_{c}+t \varphi} \varphi+\frac{\partial}{\partial \bar{u}} B\left(|u|^{2}\right)_{\mid u=u_{c}+t \varphi} \bar{\varphi}\right] d t \\
& =B\left(\left|u_{c}\right|^{2}\right)+\int_{0}^{1} d t \sum_{i+j \leq 4} \frac{1}{i ! j !} \partial_{u}^{i+1} \partial_{\bar{u}}^{j} B\left(|u|^{2}\right)_{\mid u=t \varphi} u_{c}^{i}{\overline{u_{c}}}^{j} \varphi+ \\
& \int_{0}^{1} d t \sum_{i+j \leq 4} \frac{1}{i ! j !} \partial_{u}^{i} \partial_{\bar{u}}^{j+1} B\left(|u|^{2}\right)_{\mid u=t \varphi} u_{c}^{i}{\overline{u_{c}}}^{j} \bar{\varphi}+ \\
& 5 \int_{[0,1]^{2}} d t d s(1-s)^{4} \sum_{i+j=5} \frac{1}{i ! j !} \partial_{u}^{i+1} \partial_{\bar{u}}^{j} B\left(|u|^{2}\right)_{\mid u=t \varphi+s u_{c}} u_{c}^{i}{\overline{u_{c}}}^{j} \varphi+ \\
& 5 \int_{[0,1]^{2}} d t d s(1-s)^{4} \sum_{i+j=5} \frac{1}{i ! j !} \partial_{u}^{i} \partial_{\bar{u}}^{j+1} B\left(|u|^{2}\right)_{\mid u=t \varphi+s u_{c}} u_{c}^{i} \bar{u}_{c}^{j} \bar{\varphi} .
\end{aligned}
$$

Lemma 8.2. The following statements hold.

$$
\begin{aligned}
& K=d(\omega)-\omega\left\|u_{0}\right\|_{2}^{2}+K_{2}+K_{P} \\
& K_{2}=\sum_{j} \lambda_{j}(\omega)\left|z_{j}\right|^{2}+\frac{1}{2}\left\langle\sigma_{3} \mathcal{H}_{\omega} f, \sigma_{1} f\right\rangle \\
& K_{P}=\sum_{|\mu+\nu|=3}\left\langle a_{\mu \nu}(\omega, z), 1\right\rangle z^{\mu} \bar{z}^{\nu}+\sum_{|\mu+\nu|=2} z^{\mu} \bar{z}^{\nu}\left\langle G_{\mu \nu}(\omega, z), \sigma_{3} \sigma_{1} P_{c}(\omega) f\right\rangle \\
& +\sum_{d=2}^{4}\left\langle B_{d}(\omega, z),\left(P_{c}(\omega) f\right)^{\otimes d}\right\rangle+\left\langle B_{6}(\omega, f), 1\right\rangle+\int_{\mathbb{R}^{3}} B_{5}(x, \omega, z, f(x)) f^{\otimes 5}(x) d x
\end{aligned}
$$

for $B_{6}(x, \omega, f)=B\left(\frac{\left|P_{c}(\omega) f(x)\right|^{2}}{2}\right)$, where we have what follows.

(1) $a_{\mu \nu}(\cdot, \omega, z) \in C^{\infty}\left(\mathrm{U}, H_{x}^{K, S}\left(\mathbb{R}^{3}, \mathbb{C}\right)\right)$ for any pair $(K, S)$ and a small neighborhood $\mathrm{U}$ of $\left(\omega_{0}, 0\right)$ in $\mathcal{O} \times \mathbb{C}^{m}$.

(2) $G_{\mu \nu}(\cdot, \omega, z) \in C^{\infty}\left(\mathrm{U}, H_{x}^{K, S}\left(\mathbb{R}^{3}, \mathbb{C}^{2}\right)\right)$, for $\mathrm{U}$ like in (1), possibly smaller.

(3) $B_{d}(\cdot, \omega, z) \in C^{\infty}\left(\mathrm{U}, H_{x}^{K, S}\left(\mathbb{R}^{3}, B\left(\left(\mathbb{C}^{2}\right)^{\otimes d}, \mathbb{C}\right)\right)\right)$, for $2 \leq d \leq 4$ for $\mathrm{U}$ possibly smaller.

(4) Let $^{t} \eta=(\zeta, \bar{\zeta})$ for $\zeta \in \mathbb{C}$. Then for $B_{5}(\cdot, \omega, z, \eta)$ we have

$$
\text { for any } l,\left\|\nabla_{\omega, z, \bar{z}, \zeta, \bar{\zeta}}^{l} B_{5}(\omega, z, \eta)\right\|_{H_{x}^{K, S}\left(\mathbb{R}^{3}, B\left(\left(\mathbb{C}^{2}\right)^{\otimes 5}, \mathbb{C}\right)\right.} \leq C_{l} .
$$

(5) We have $a_{\mu \nu}=\bar{a}_{\nu \mu}, G_{\mu \nu}=-\sigma_{1} \bar{G}_{\nu \mu}$. 
Proof. The expansion for $K$ is a consequence of well know cancelations. (1)-(4) follow from (8.7) and elementary calculus. (5) follows from the fact that $K(U)$ is real valued for $\bar{U}=\sigma_{1} U$.

We set $\delta_{j}$ be for $j \in\{1, \ldots m\}$ the multi index $\delta_{j}=\left(\delta_{1 j}, \ldots, \delta_{m j}\right)$. Let $\lambda_{j}^{0}=$ $\lambda_{j}\left(\omega_{0}\right)$ and $\lambda^{0}=\left(\lambda_{1}^{0}, \cdots, \lambda_{m}^{0}\right)$.

Lemma 8.3. Let $H=K \circ \mathcal{F}_{1}$. Then, at $e^{i \sigma_{3} \vartheta} \Phi_{\omega_{0}}$ we have the expansion

$$
H=d\left(\omega_{0}\right)-\omega_{0}\left\|u_{0}\right\|_{2}^{2}+\psi\left(\|f\|_{2}^{2}\right)+H_{2}^{(1)}+\mathcal{R}^{(1)}
$$

for $\omega=\omega_{0}$, where the following holds.

(1) We have for $r=1$

$$
H_{2}^{(r)}=\sum_{\substack{|\mu+\nu|=2 \\ \lambda^{0} \cdot(\mu-\nu)=0}} a_{\mu \nu}^{(r)}\left(\|f\|_{2}^{2}\right) z^{\mu} \bar{z}^{\nu}+\frac{1}{2}\left\langle\sigma_{3} \mathcal{H}_{\omega_{0}} f, \sigma_{1} f\right\rangle .
$$

(2) We have $\mathcal{R}^{(1)}=\widetilde{\mathcal{R}^{(1)}}+\widetilde{\mathcal{R}^{(2)}}$, with $\widetilde{\mathcal{R}^{(1)}}=$

$$
\begin{aligned}
& =\sum_{\substack{|\mu+\nu|=2 \\
\lambda^{0} \cdot(\mu-\nu) \neq 0}} a_{\mu \nu}^{(1)}\left(\|f\|_{2}^{2}\right) z^{\mu} \bar{z}^{\nu}+\sum_{|\mu+\nu|=1} z^{\mu} \bar{z}^{\nu}\left\langle\sigma_{1} \sigma_{3} G_{\mu \nu}\left(\|f\|_{2}^{2}\right), f\right\rangle, \\
& \widetilde{\mathcal{R}^{(2)}}=\sum_{|\mu+\nu|=3} z^{\mu} \bar{z}^{\nu} \int_{\mathbb{R}^{3}} a_{\mu \nu}\left(x, z, f, f(x),\|f\|_{2}^{2}\right) d x \\
& +\sum_{|\mu+\nu|=2} z^{\mu} \bar{z}^{\nu} \int_{\mathbb{R}^{3}}\left[\sigma_{1} \sigma_{3} G_{\mu \nu}\left(x, z, f, f(x),\|f\|_{2}^{2}\right)\right]^{*} f(x) d x \\
& +\sum_{j=2}^{5} \mathcal{R}_{j}^{(1)}+\int_{\mathbb{R}^{3}} B\left(|f(x)|^{2} / 2\right) d x+\widehat{\mathcal{R}}_{2}^{(1)}\left(z, f,\|f\|_{2}^{2}\right) \\
& \text { with } \mathcal{R}_{j}^{(1)}=\int_{\mathbb{R}^{3}} F_{j}\left(x, z, f, f(x),\|f\|_{2}^{2}\right) f^{\otimes j}(x) d x .
\end{aligned}
$$

(3) We have $F_{2}(x, 0,0,0,0)=0$.

(4) $\psi(s)$ is smooth with $\psi(0)=\psi^{\prime}(0)=0$.

(5) $A t\|f\|_{2}=0$ with $r=1$

$$
\begin{aligned}
& a_{\mu \nu}^{(r)}(0)=0 \text { for }|\mu+\nu|=2 \text { with }(\mu, \nu) \neq\left(\delta_{j}, \delta_{j}\right) \text { for all } j, \\
& a_{\delta_{j} \delta_{j}}^{(r)}(0)=\lambda_{j}\left(\omega_{0}\right) \text {, where } \delta_{j}=\left(\delta_{1 j}, \ldots, \delta_{m j}\right), \\
& G_{\mu \nu}(0)=0 \text { for }|\mu+\nu|=1
\end{aligned}
$$

These $a_{\mu \nu}^{(r)}(\varrho)$ and $G_{\mu \nu}(x, \varrho)$ are smooth in all variables with $G_{\mu \nu}(\cdot, \varrho) \in$ $C^{\infty}\left(\mathbb{R}, H_{x}^{K, S}\left(\mathbb{R}^{3}, \mathbb{C}^{2}\right)\right)$ for all $(K, S)$. 
(6) We have for all indexes and for $r=1$

$$
a_{\mu \nu}^{(r)}=\bar{a}_{\nu \mu}^{(r)}, \quad a_{\mu \nu}=\bar{a}_{\nu \mu}, \quad G_{\mu \nu}=-\sigma_{1} \bar{G}_{\nu \mu} .
$$

(7) Let ${ }^{t} \eta=(\zeta, \bar{\zeta})$ for $\zeta \in \mathbb{C}$. For all $\left(K, S, K^{\prime}, S^{\prime}\right)$ there is a neighborhood $\mathcal{U}^{-K^{\prime},-S^{\prime}}$ of $\{(0,0)\}$ in $\widetilde{\mathcal{P}}^{-K^{\prime},-S^{\prime}}$, see $(7.40)$, such that we have, for $a_{\mu \nu}(x, z, f, \eta, \varrho)$ with $(z, f, \zeta, \varrho) \in \mathcal{U}^{-K^{\prime},-S^{\prime}} \times \mathbb{C} \times \mathbb{R}$

$$
\left\|\nabla_{z, \bar{z}, \zeta, \bar{\zeta}, f, \varrho}^{l} a_{\mu \nu}\right\|_{H_{x}^{K, S}\left(\mathbb{R}^{3}, \mathbb{C}\right)} \leq C_{l} \text { for all } l .
$$

(8) Possibly restricting $\mathcal{U}^{-K^{\prime},-S^{\prime}}$, we have also, for $G_{\mu \nu}(x, z, f, g, \varrho)$,

$$
\left\|\nabla_{z, \bar{z}, \zeta, \bar{\zeta}, f, \varrho}^{l} G_{\mu \nu}\right\|_{H_{x}^{K, S}\left(\mathbb{R}^{3}, \mathbb{C}^{2}\right)} \leq C_{l} \text { for all } l .
$$

(9) Restricting $\mathcal{U}^{-K^{\prime},-S^{\prime}}$ further, we have also, for $F_{j}(x, z, f, g, \varrho)$,

$$
\left\|\nabla_{z, \bar{z}, \zeta, \bar{\zeta}, f, \varrho}^{l} F_{j}\right\|_{H_{x}^{K, S}\left(\mathbb{R}^{3}, B\left(\left(\mathbb{C}^{2}\right)^{\otimes j}, \mathbb{C}\right)\right)} \leq C_{l} \text { for all } l .
$$

(10) Restricting $\mathcal{U}^{-K^{\prime},-S^{\prime}}$ further, we have $\widehat{\mathcal{R}}_{2}^{(1)}(z, f, \varrho) \in C^{\infty}\left(\mathcal{U}^{-K^{\prime},-S^{\prime}} \times \mathbb{R}, \mathbb{R}\right)$ with

$$
\left|\widehat{\mathcal{R}}_{2}^{(1)}(z, f, \varrho)\right| \leq C\left(|z|+|\varrho|+\|f\|_{H^{-K^{\prime},-S^{\prime}}}\right)\|f\|_{H^{-K^{\prime},-S^{\prime}}}^{2}
$$

Proof. By $\mathcal{F}_{1}\left(\Phi_{\omega_{0}}\right)=\Phi_{\omega_{0}}, K^{\prime}\left(\Phi_{\omega_{0}}\right)=0$ and $\left\|\mathcal{F}_{1}(U)-U\right\|_{\mathcal{P}^{K, S}} \lesssim\|R\|_{L^{2}}^{2}$ we conclude $H^{\prime}\left(\Phi_{\omega_{0}}\right)=0$ and $H^{\prime \prime}\left(\Phi_{\omega_{0}}\right)=K^{\prime \prime}\left(\Phi_{\omega_{0}}\right)$. In particular, this yields the formula for $H_{2}^{(1)}$ for $\|f\|_{2}=0$. The other terms are obtained by substituting in (8.8) the formulas (7.59). By $\left\langle\sigma_{3} f, \sigma_{1} f\right\rangle=0$ we have $\left\langle\sigma_{3} \mathcal{H}_{\omega_{0}+\delta \omega} f, \sigma_{1} f\right\rangle=$ $\left\langle\sigma_{3} \mathcal{H}_{\omega_{0}} f, \sigma_{1} f\right\rangle+\widetilde{F}_{2}$ where $\widetilde{F}_{2}$ can be absorbed in $j=2$ in $(8.10) . \psi\left(\|f\|_{2}^{2}\right)$ arises from $d\left(\omega \circ \mathcal{F}_{1}\right)-\omega \circ \mathcal{F}_{1}\left\|u_{0}\right\|_{2}^{2}$. Other terms coming from the latter end up in (8.10): in particular there are no monomials $\|f\|_{2}^{j} z^{\mu} \bar{z}^{\nu}\langle G, f\rangle^{i}$ with $|\mu+\nu|+i=1$, because of (7.60) (applied for $\omega=\omega_{0}$ ).

\section{Canonical transformations}

Our goal in this section is to prove the following result.

Theorem 9.1. For any integer $r \geq 2$ there are a neighborhood $\mathcal{U}^{1,0}$ of $\{(0,0)\}$ in $\widetilde{\mathcal{P}}^{1,0}$, see (7.40), and a smooth canonical transformation $\mathcal{T}_{r}: \mathcal{U}^{1,0} \rightarrow \widetilde{\mathcal{P}}^{1,0}$ s.t.

$$
H^{(r)}:=H \circ \mathcal{T}_{r}=d\left(\omega_{0}\right)-\omega_{0}\left\|u_{0}\right\|_{2}^{2}+\psi\left(\|f\|_{2}^{2}\right)+H_{2}^{(r)}+Z^{(r)}+\mathcal{R}^{(r)},
$$

where:

(i) $H_{2}^{(r)}=H_{2}^{(2)}$ for $r \geq 2$, is of the form (8.9) where $a_{\mu \nu}^{(r)}\left(\|f\|_{2}\right)$ satisfy (8.11)-(8.12); 
(ii) $Z^{(r)}$ is in normal form, in the sense of Definition 9.3 below, with monomials of degree $\leq r$ whose coefficients satisfy (8.12);

(iii) the transformation $\mathcal{T}_{r}$ is of the form (see below) (9.9)-(9.10) and satisfies (9.12)-(9.13) for $M_{0}=1$;

(iv) we have $\mathcal{R}^{(r)}=\sum_{d=0}^{6} \mathcal{R}_{d}^{(r)}$ with the following properties:

(iv.0) for all $\left(K, S, K^{\prime}, S^{\prime}\right)$ there is a neighborhood $\mathcal{U}^{-K^{\prime},-S^{\prime}}$ of $\{(0,0)\}$ in $\widetilde{\mathcal{P}}^{-K^{\prime},-S^{\prime}}$ such that

$$
\mathcal{R}_{0}^{(r)}=\sum_{|\mu+\nu|=r+1} z^{\mu} \bar{z}^{\nu} \int_{\mathbb{R}^{3}} a_{\mu \nu}^{(r)}\left(x, z, f, f(x),\|f\|_{2}^{2}\right) d x
$$

and for $a_{\mu \nu}^{(r)}(z, f, \eta, \varrho)$ with ${ }^{t} \eta=(\zeta, \bar{\zeta}), \zeta \in \mathbb{C}$ we have for $(z, f) \in$ $\mathcal{U}^{-K^{\prime},-S^{\prime}}$ and $|\varrho| \leq 1$

$$
\left\|\nabla_{z, \bar{z}, \zeta, \bar{\zeta}, f, \varrho}^{l} a_{\mu \nu}^{(r)}(\cdot, z, f, \eta, \varrho)\right\|_{H^{K, S}\left(\mathbb{R}^{3}, \mathbb{C}\right)} \leq C_{l} \text { for all } l ;
$$

(iv.1) possibly taking $\mathcal{U}^{-K^{\prime},-S^{\prime}}$ smaller, we have

$$
\begin{aligned}
& \mathcal{R}_{1}^{(r)}=\sum_{|\mu+\nu|=r} z^{\mu} \bar{z}^{\nu} \int_{\mathbb{R}^{3}}\left[\sigma_{1} \sigma_{3} G_{\mu \nu}^{(r)}\left(x, z, f, f(x),\|f\|_{2}^{2}\right)\right]^{*} f(x) d x \\
& \text { with }\left\|\nabla_{z, \bar{z}, \zeta, \bar{\zeta}, f, \varrho}^{l} G_{\mu \nu}^{(r)}(\cdot, z, f, \eta, \varrho)\right\|_{H^{K, S}\left(\mathbb{R}^{3}, \mathbb{C}^{2}\right)} \leq C_{l} \text { for all } l ;
\end{aligned}
$$

(iv.2-5) possibly taking $\mathcal{U}^{-K^{\prime},-S^{\prime}}$ smaller, we have for $2 \leq d \leq 5$,

$$
\mathcal{R}_{d}^{(r)}=\int_{\mathbb{R}^{3}} F_{d}^{(r)}\left(x, z, f, f(x),\|f\|_{2}^{2}\right) f^{\otimes d}(x) d x+\widehat{\mathcal{R}}_{d}^{(r)},
$$

with for any $l$

$$
\left\|\nabla_{z, \bar{z}, \zeta, \bar{\zeta}, f, \varrho}^{l} F_{d}^{(r)}(\cdot, z, f, \eta, \varrho)\right\|_{H^{K, S}\left(\mathbb{R}^{3}, B\left(\left(\mathbb{C}^{2}\right)^{\otimes d}, \mathbb{C}\right)\right.} \leq C_{l},
$$

with $F_{2}^{(r)}(x, 0,0,0,0)=0$ and with $\widetilde{\mathcal{R}}_{d}^{(r)}\left(z, f,\|f\|_{2}^{2}\right)$ s.t.

$$
\begin{aligned}
& \widehat{\mathcal{R}}_{d}^{(r)}(z, f, \varrho) \in C^{\infty}\left(\mathcal{U}^{-K^{\prime},-S^{\prime}} \times \mathbb{R}, \mathbb{R}\right), \\
& \left|\widehat{\mathcal{R}}_{d}^{(r)}(z, f, \varrho)\right| \leq C\|f\|_{H^{-K^{\prime},-S^{\prime}}}^{d}, \\
& \left|\widehat{\mathcal{R}}_{2}^{(r)}(z, f, \varrho)\right| \leq C\left(|z|+|\varrho|+\|f\|_{H^{-K^{\prime},-S^{\prime}}}\right)\|f\|_{H^{-K^{\prime},-S^{\prime}}}^{2}
\end{aligned}
$$

(iv.6) $\mathcal{R}_{6}^{(r)}=\int_{\mathbb{R}^{3}} B\left(|f(x)|^{2} / 2\right) d x$.

We develop the proof in the following subsections. The basic ideas are classical. However we need to develop a number of tools, along the lines of [BC]. The situation here is more complicated than in $[\mathrm{BC}]$ because of the dependence of the coefficients on $\|f\|_{2}$. 


\subsection{Lie transform}

We consider functions

$$
\chi=\sum_{|\mu+\nu|=M_{0}+1} b_{\mu \nu}\left(\|f\|_{2}^{2}\right) z^{\mu} \bar{z}^{\nu}+\sum_{|\mu+\nu|=M_{0}} z^{\mu} \bar{z}^{\nu}\left\langle\sigma_{1} \sigma_{3} B_{\mu \nu}\left(\|f\|_{2}^{2}\right), f\right\rangle
$$

where $b_{\mu \nu}(\varrho) \in C^{\infty}\left(\mathbb{R}_{\varrho}, \mathbb{C}\right)$ and $B_{\mu \nu}(x, \varrho) \in C^{\infty}\left(\mathbb{R}, P_{c}\left(\omega_{0}\right) H_{x}^{k, s}\left(\mathbb{R}^{3}, \mathbb{C}^{2}\right)\right)$ for all $k$ and $s$. Assume

$$
b_{\mu \nu}=\bar{b}_{\nu \mu} \text { and } \sigma_{1} B_{\mu \nu}=-\bar{B}_{\nu \mu} \text { for all indexes. }
$$

We set for $K>0$ and $S>0$ fixed and large set

$$
\|\chi\|=\left\|\chi\left(\|f\|_{2}^{2}\right)\right\|=\sum\left|b_{\mu \nu}\left(\|f\|_{2}^{2}\right)\right|+\sum\left\|B_{\mu \nu}\left(\|f\|_{2}^{2}\right)\right\|_{H^{K, S}} .
$$

Denote by $\phi^{t}$ the flow of the Hamiltonian vector field $X_{\chi}$ ( from now on with respect to $\Omega_{0}$ and only in $\left.(z, f)\right)$. The Lie transform $\phi=\left.\phi^{t}\right|_{t=1}$ is defined in a sufficiently small neighborhood of the origin and is a canonical transformation.

Lemma 9.2. Consider the $\chi$ in (9.6) and its Lie transform $\phi$. Set $\left(z^{\prime}, f^{\prime}\right)=$ $\phi(z, f)$. Then there are $\mathcal{G}(z, f, \varrho), \Gamma(z, f, \varrho), \Gamma_{0}(z, f, \rho)$ and $\Gamma_{1}(z, f, \rho)$ with the following properties.

(1) $\Gamma \in C^{\infty}\left(\mathcal{U}^{-K^{\prime},-S^{\prime}}, \mathbb{C}^{m}\right), \Gamma_{0}, \Gamma_{1} \in C^{\infty}\left(\mathcal{U}^{-K^{\prime},-S^{\prime}}, \mathbb{R}\right)$, with $\mathcal{U}^{-K^{\prime},-S^{\prime}} \subset$ $\mathbb{C}^{m} \times H_{c}^{-K^{\prime},-S^{\prime}}\left(\omega_{0}\right) \times \mathbb{R}$ an appropriately small neighborhood of the origin.

(2) $\mathcal{G} \in C^{\infty}\left(\mathcal{U}^{-K^{\prime},-S^{\prime}}, H_{c}^{K, S}\left(\omega_{0}\right)\right)$ for any $K, S$.

(3) The transformation $\phi$ is of the following form:

$$
\begin{gathered}
z^{\prime}=z+\Gamma\left(z, f,\|f\|_{2}^{2}\right), \\
f^{\prime}=e^{\mathrm{i} \Gamma_{0}\left(z, f,\|f\|_{2}^{2}\right) P_{c}\left(\omega_{0}\right) \sigma_{3}} f+\mathcal{G}\left(z, f,\|f\|_{2}^{2}\right) .
\end{gathered}
$$

(4) We have

$$
\begin{gathered}
\left\|f^{\prime}\right\|_{2}^{2}=\|f\|_{2}^{2}+\Gamma_{1}\left(z, f,\|f\|_{2}^{2}\right), \\
\left|\Gamma_{1}\left(z, f,\|f\|_{2}^{2}\right)\right| \leq \\
C|z|^{M_{0}-1}\left(|z|^{M_{0}+2}+|z|^{2}\|f\|_{H^{-K^{\prime},-S^{\prime}}}+\|f\|_{H^{-K^{\prime},-S^{\prime}}}^{3}\right) .
\end{gathered}
$$

(5) There are constants $c_{K^{\prime}, S^{\prime}}$ and $c_{K, S, K^{\prime}, S^{\prime}}$ such that

$$
\begin{aligned}
& \left|\Gamma\left(z, f,\|f\|_{2}^{2}\right)\right| \leq c_{K^{\prime}, S^{\prime}}(\|\chi\|+(9.12))|z|^{M_{0}-1}\left(|z|+\|f\|_{H^{-K^{\prime},-S^{\prime}}}\right), \\
& \left\|\mathcal{G}\left(z, f,\|f\|_{2}^{2}\right)\right\|_{H^{K, S}} \leq c_{K, S, K^{\prime}, S^{\prime}}(\|\chi\|+(9.12))|z|^{M_{0}}, \\
& \left|\Gamma_{0}\left(z, f,\|f\|_{2}^{2}\right)\right| \leq c_{K^{\prime}, S^{\prime}}|z|^{M_{0}-1}\left(|z|+\|f\|_{H^{-K^{\prime},-S^{\prime}}}\right)^{2} .
\end{aligned}
$$


(6) We have

$$
e^{\mathrm{i} \Gamma_{0} P_{c}\left(\omega_{0}\right) \sigma_{3}}=e^{\mathrm{i} \Gamma_{0} \sigma_{3}}+T\left(\Gamma_{0}\right),
$$

where $T(r) \in C^{\infty}\left(\mathbb{R}, B\left(H^{-K^{\prime},-S^{\prime}}, H^{K, S}\right)\right)$ for all $\left(K, S, K^{\prime}, S^{\prime}\right)$, with norm $\|T(r)\|_{B\left(H^{\left.-K^{\prime},-S^{\prime}, H^{K, S}\right)}\right.} \leq C\left(K, S, K^{\prime}, S^{\prime}\right)|r|$. More specifically, the range of $T(r)$ is $R(T(r)) \subseteq L_{d}^{2}(\mathcal{H})+L_{d}^{2}\left(\mathcal{H}^{*}\right), L_{d}^{2}$ defined two lines after (3.11).

Proof. Claim (6) can be proved independently of the properties of $\Gamma_{0}$. Recall that $P_{c}(\omega)=1-P_{d}(\omega)$, see below (3.11), with $P_{d}(\omega)$ smoothing and of finite rank. Exploiting $\sigma_{3} P_{d}(\omega)=P_{d}^{*}(\omega) \sigma_{3}$ it is elementary to prove

$$
\begin{aligned}
& e^{\mathrm{i} \Gamma_{0} P_{c}\left(\omega_{0}\right) \sigma_{3}}=e^{\mathrm{i} \Gamma_{0} \sigma_{3}}+T\left(\Gamma_{0}\right) \text { with } T\left(\Gamma_{0}\right)=-\mathrm{i} \sin \left(\Gamma_{0}\right) P_{d}\left(\omega_{0}\right) \sigma_{3}+ \\
& +\sum_{n=2}^{\infty} \frac{\left(\mathrm{i} \Gamma_{0}\right)^{n}}{n !} \sum_{j=1}^{\left[\frac{n}{2}\right]}\left(\begin{array}{c}
{\left[\frac{n}{2}\right]} \\
j
\end{array}\right) K^{j}\left(P_{c}\left(\omega_{0}\right) \sigma_{3}\right)^{\varepsilon(n)}
\end{aligned}
$$

with $K=P_{d}\left(\omega_{0}\right) P_{d}^{*}\left(\omega_{0}\right)-P_{d}\left(\omega_{0}\right)-P_{d}^{*}\left(\omega_{0}\right)$ and $\varepsilon(n)=\frac{1-(-1)^{n}}{2} . T\left(\Gamma_{0}\right)$ has the properties of Claim (6).

In the sequel we prove Claims (1)-(5). Set $\varrho=\|f\|_{2}^{2}$. For $b_{\mu \nu}^{\prime}$ and $B_{\mu \nu}^{\prime}$ derivatives with respect to $\varrho$, summing on repeated indexes, consider

$$
\gamma(z, f, \varrho):=2\left(b_{\mu \nu}^{\prime}(\varrho) z^{\mu} \bar{z}^{\nu}+\left\langle\sigma_{1} \sigma_{3} B_{\mu \nu}^{\prime}(\varrho), f\right\rangle z^{\mu} \bar{z}^{\nu}\right) .
$$

For $\sigma_{1} f=\bar{f}$, then $\gamma(z, f, \varrho) \in \mathbb{R}$ by $(9.7)$. We set up the following system:

$$
\begin{aligned}
& \mathrm{i} \dot{z}_{j}=\sum_{|\mu+\nu|=M_{0}+1} \nu_{j} \frac{z^{\mu} \bar{z}^{\nu}}{\bar{z}_{j}} b_{\mu \nu}(\varrho)+\sum_{|\mu+\nu|=M_{0}} \nu_{j} \frac{z^{\mu} \bar{z}^{\nu}}{\bar{z}_{j}}\left\langle\sigma_{1} \sigma_{3} B_{\mu \nu}(\varrho), f\right\rangle \\
& \mathrm{i} \dot{f}=\sum_{|\mu+\nu|=M_{0}} z^{\mu} \bar{z}^{\nu} B_{\mu \nu}(\varrho)+\gamma(z, f, \varrho) P_{c}\left(\omega_{0}\right) \sigma_{3} f \\
& \dot{\varrho}=-2 \mathrm{i}\left\langle\sum_{|\mu+\nu|=M_{0}} z^{\mu} \bar{z}^{\nu} B_{\mu \nu}(\varrho)+\gamma(z, f, \varrho)\left(P_{c}\left(\omega_{0}\right)-P_{c}^{*}\left(\omega_{0}\right)\right) \sigma_{3} f, \sigma_{1} f\right\rangle,
\end{aligned}
$$

where in the last equation we exploited $\left\langle\sigma_{3} f, \sigma_{1} f\right\rangle=0$. By (9.7) the flow leaves the set with $\sigma_{1} f=\bar{f}$ and $\varrho \in \mathbb{R}$ invariant. In particular, the set where $\varrho=\|f\|_{2}^{2}$ is invariant under the flow of (9.17). In a neighborhood of 0 the lifespan of the solutions is larger than 1. (9.9) can always been written. For $\gamma$ defined in (9.16), we have

$$
f(t)=e^{-\mathrm{i} \int_{0}^{t} \gamma d s P_{c}\left(\omega_{0}\right) \sigma_{3}} f-\sum_{|\mu+\nu|=M_{0}} \mathrm{i} \int_{0}^{t} z^{\mu} \bar{z}^{\nu} e^{\mathrm{i} \int_{s}^{t} \gamma d s^{\prime} P_{c}\left(\omega_{0}\right) \sigma_{3}} B_{\mu \nu} d s .
$$

This yields (9.10). We can always write

$$
\varrho^{\prime}=\varrho+\Gamma_{1}(z, f, \varrho) .
$$


This yields (9.11). Claims (1)-(2) follow from the regularity of the flow of (9.17) on the initial data. By (9.17) we get

$$
\begin{aligned}
& |\varrho(t)-\varrho| \leq C \sup _{0 \leq t^{\prime} \leq t}\left|z\left(t^{\prime}\right)\right|^{M_{0}-1}\left(\left|z\left(t^{\prime}\right)\right|^{M_{0}+2}+\right. \\
& \left.+\left|z\left(t^{\prime}\right)\right|^{2}\left\|f\left(t^{\prime}\right)\right\|_{H^{-K^{\prime},-S^{\prime}}}+\left\|f\left(t^{\prime}\right)\right\|_{H^{-K^{\prime},-S^{\prime}}}^{3}\right) .
\end{aligned}
$$

Similarly we have

$$
\begin{aligned}
& |z(t)-z| \leq C \sup _{0 \leq t^{\prime} \leq t}\left|z\left(t^{\prime}\right)\right|^{M_{0}-1}\left\|\chi\left(\varrho\left(t^{\prime}\right)\right)\right\|\left(\left|z\left(t^{\prime}\right)\right|+\left\|f\left(t^{\prime}\right)\right\|_{H^{-K^{\prime},-S^{\prime}}}\right), \\
& \left\|\int_{0}^{t} z^{\mu} \bar{z}^{\nu} e^{\mathrm{i} \int_{s}^{t} \gamma d s^{\prime} P_{c}\left(\omega_{0}\right) \sigma_{3}} B_{\mu \nu} d s\right\|_{H^{K, S}} \leq C \sup _{0 \leq t^{\prime} \leq t}\left|z\left(t^{\prime}\right)\right|^{M_{0}}\left\|\chi\left(\varrho\left(t^{\prime}\right)\right)\right\| .
\end{aligned}
$$

Then $|z(t)| \approx|z|+\|f\|_{H^{-K^{\prime},-S^{\prime}}}$ with in particular $|z(t)| \approx|z|$ when $M_{0}>1$. By Claim (6) and by the fact that the exponent $\Gamma_{0}(z, f, \varrho)$ in (9.10) is a uniformly bounded function, we get $\|f(t)\|_{H^{-K^{\prime},-S^{\prime}}} \approx|z|+\|f\|_{H^{-K^{\prime},-S^{\prime}}}$. Then

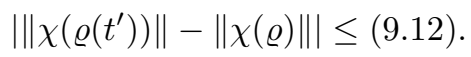

This implies that the right hand sides of (9.19)-(9.21) are bounded by the bounds of $\Gamma_{1}, \Gamma$ and $\mathcal{G}$ in the statement. This yields the desired bounds on $\Gamma_{1}$, $\Gamma$ and $\mathcal{G}$. The bound on $\Gamma_{0}$ follows from

$$
\begin{aligned}
& \left|\int_{0}^{t} \gamma\left(t^{\prime}\right) d t^{\prime}\right| \leq C \sup _{0 \leq t^{\prime} \leq t}\left|z\left(t^{\prime}\right)\right|^{M_{0}}\left(\left|z\left(t^{\prime}\right)\right|+\left\|f\left(t^{\prime}\right)\right\|_{H^{-K^{\prime},-S^{\prime}}}\right) \\
& \leq C_{1}|z|^{M_{0}-1}\left(|z|+\|f\|_{H^{-K^{\prime},-S^{\prime}}}\right)^{2} .
\end{aligned}
$$

\subsection{Normal form}

Recall the notation $\lambda_{j}^{0}=\lambda_{j}\left(\omega_{0}\right)$ and $\delta_{j}=\left(\delta_{1 j}, \ldots, \delta_{m j}\right)$, see before Lemma 8.3. Let $\mathcal{H}=\mathcal{H}_{\omega_{0}} P_{c}\left(\mathcal{H}_{\omega_{0}}\right)$. For $r \geq 1$, using the coefficients in (8.9) of the $H_{2}^{(r)}$ in Theorem 9.1, let

$$
\lambda_{j}^{(r)}=\lambda_{j}^{(r)}\left(\|f\|_{2}^{2}\right)=\lambda_{j}\left(\omega_{0}\right)+a_{\delta_{j} \delta_{j}}^{(r)}\left(\|f\|_{2}^{2}\right), \quad \lambda^{(r)}=\left(\lambda_{1}^{(r)}, \cdots, \lambda_{m}^{(r)}\right) .
$$

Definition 9.3. A function $Z(z, f)$ is in normal form if it is of the form

$$
Z=Z_{0}+Z_{1}
$$

where we have finite sums of the following types:

$$
Z_{1}=\sum_{\left|\lambda^{0} \cdot(\nu-\mu)\right|>\omega_{0}} z^{\mu} \bar{z}^{\nu}\left\langle\sigma_{1} \sigma_{3} G_{\mu \nu}\left(\|f\|_{2}^{2}\right), f\right\rangle
$$


with $G_{\mu \nu}(x, \varrho) \in C^{\infty}\left(\mathbb{R}_{\varrho}, H_{x}^{K, S}\right)$ for all $K, S$;

$$
Z_{0}=\sum_{\lambda^{0} \cdot(\mu-\nu)=0} a_{\mu, \nu}\left(\|f\|_{2}^{2}\right) z^{\mu} \bar{z}^{\nu}
$$

and $a_{\mu, \nu}(\varrho) \in C^{\infty}\left(\mathbb{R}_{\varrho}, \mathbb{C}\right)$. We will always assume the symmetries (8.12).

For an $H_{2}^{(r)}$ as in (8.9) let $H_{2}^{(r)}=D_{2}^{(r)}+\left(H_{2}^{(r)}-D_{2}^{(r)}\right)$ where

$$
D_{2}^{(r)}=\sum_{j=1}^{m} \lambda_{j}^{(r)}\left(\|f\|_{2}^{2}\right)\left|z^{j}\right|+\frac{1}{2}\left\langle\sigma_{3} \mathcal{H}_{\omega_{0}} f, \sigma_{1} f\right\rangle .
$$

In the following formulas we set $\lambda_{j}=\lambda_{j}^{(r)}, \lambda=\lambda^{(r)}$ and $D_{2}=D_{2}^{(r)}$. We recall $\left(\lambda_{j}^{\prime}(\varrho)\right.$ is the derivative in $\left.\varrho\right)$ that by $(5.3)$, summing on repeated indexes,

$$
\begin{aligned}
& \left\{D_{2}, F\right\}:=d D_{2}\left(X_{F}\right)=\partial_{j} D_{2}\left(X_{F}\right)_{j}+\partial_{\bar{j}} D_{2}\left(X_{F}\right)_{\bar{j}}+\left\langle\nabla_{f} D_{2},\left(X_{F}\right)_{f}\right\rangle \\
& =-\mathrm{i} \partial_{j} D_{2} \partial_{\bar{j}} F+\mathrm{i} \partial_{\bar{j}} D_{2} \partial_{j} F-\mathrm{i}\left\langle\nabla_{f} D_{2}, \sigma_{3} \sigma_{1} \nabla_{f} F\right\rangle= \\
& \mathrm{i} \lambda_{j} z_{j} \partial_{j} F-\mathrm{i} \lambda_{j} \bar{z}_{j} \partial_{\bar{j}} F+\mathrm{i}\left\langle\mathcal{H} f, \nabla_{f} F\right\rangle+2 \mathrm{i} \lambda_{j}^{\prime}\left(\|f\|_{2}^{2}\right)\left|z_{j}\right|^{2}\left\langle f, \sigma_{3} \nabla_{f} F\right\rangle .
\end{aligned}
$$

In particular, we have, for $G=G(x)$, (we use $\left.\sigma_{1} \mathrm{i} \sigma_{2}=\sigma_{3}\right)$

$$
\begin{aligned}
& \left\{D_{2}, z^{\mu} \bar{z}^{\nu}\right\}=\mathrm{i} \lambda \cdot(\mu-\nu) z^{\mu} \bar{z}^{\nu}, \\
& \left\{D_{2},\left\langle\sigma_{1} \sigma_{3} G, f\right\rangle\right\}=-\mathrm{i}\left\langle f, \sigma_{1} \sigma_{3} \mathcal{H} G\right\rangle-2 \mathrm{i} \sum_{j=1}^{m} \lambda_{j}^{\prime}\left|z_{j}\right|^{2}\left\langle\sigma_{1} f, G\right\rangle, \\
& \left\{D_{2}, \frac{1}{2}\|f\|_{2}^{2}\right\}=\left\{D_{2}, \frac{1}{2}\left\langle f, \sigma_{1} f\right\rangle\right\}=\mathrm{i}\left\langle\mathcal{H} f, \sigma_{1} f\right\rangle=-\mathrm{i}\left\langle\beta^{\prime}\left(\phi^{2}\right) \phi^{2} \sigma_{3} f, f\right\rangle .
\end{aligned}
$$

In the sequel we will assume (and prove) that $\|f\|_{2}$ is small. We will consider only $|\mu+\nu| \leq 2 N+3$. Then, $\lambda^{0} \cdot(\mu-\nu) \neq 0$ implies $\left|\lambda^{0} \cdot(\mu-\nu)\right| \geq c>0$ for some fixed $c$, and so we can assume also $|\lambda \cdot(\mu-\nu)| \geq c / 2$. Similarly $\left|\lambda^{0} \cdot(\mu-\nu)\right|<\omega_{0}$ (resp. $\left.\left|\lambda^{0} \cdot(\mu-\nu)\right|>\omega_{0}\right)$ will be assumed equivalent to $|\lambda \cdot(\mu-\nu)|<\omega_{0}$ (resp. $\left.|\lambda \cdot(\mu-\nu)|>\omega_{0}\right)$.

Lemma 9.4. Consider

$$
K=\sum_{|\mu+\nu|=M_{0}+1} k_{\mu \nu}\left(\|f\|_{2}^{2}\right) z^{\mu} \bar{z}^{\nu}+\sum_{|\mu+\nu|=M_{0}} z^{\mu} \bar{z}^{\nu}\left\langle\sigma_{1} \sigma_{3} K_{\mu \nu}\left(\|f\|_{2}^{2}\right), f\right\rangle .
$$

Suppose that all the terms in (9.31) are not in normal form and that the symmetries (8.12) hold. Consider

$$
\begin{aligned}
\chi & =\sum_{|\mu+\nu|=M_{0}+1} \frac{k_{\mu \nu}\left(\|f\|_{2}^{2}\right)}{\mathrm{i} \lambda \cdot(\nu-\mu)} z^{\mu} \bar{z}^{\nu} \\
& -\sum_{|\mu+\nu|=M_{0}} z^{\mu} \bar{z}^{\nu}\left\langle\sigma_{1} \sigma_{3} \frac{1}{\mathrm{i}(\lambda \cdot(\mu-\nu)-\mathcal{H})} K_{\mu \nu}\left(\|f\|_{2}^{2}\right), f\right\rangle .
\end{aligned}
$$


Then we have

$$
\left\{\chi, D_{2}\right\}=K+L
$$

with, summing on repeated indexes,

$$
\begin{aligned}
& L=2 \frac{k_{\mu \nu}^{\prime}}{(\mu-\nu) \cdot \lambda} z^{\mu} \bar{z}^{\nu}\left\langle\beta^{\prime}\left(\phi^{2}\right) \phi^{2} \sigma_{3} f, f\right\rangle \\
& +2 \lambda_{j}^{\prime} z^{\mu} \bar{z}^{\nu}\left|z_{j}\right|^{2}\left\langle\sigma_{1} f, \frac{1}{(\mu-\nu) \cdot \lambda-\mathcal{H}} K_{\mu \nu}\right\rangle- \\
& 2 \lambda^{\prime} \cdot(\mu-\nu) z^{\mu} \bar{z}^{\nu}\left|z_{j}\right|^{2}\left\langle\sigma_{1} f, \frac{1}{((\mu-\nu) \cdot \lambda-\mathcal{H})^{2}} K_{\mu \nu}\right\rangle\left\langle\beta^{\prime}\left(\phi^{2}\right) \phi^{2} \sigma_{3} f, f\right\rangle \\
& +2 z^{\mu} \bar{z}^{\nu}\left\langle f, \sigma_{3} \sigma_{1} \frac{1}{(\mu-\nu) \cdot \lambda-\mathcal{H}} K_{\mu \nu}^{\prime}\right\rangle\left\langle\beta^{\prime}\left(\phi^{2}\right) \phi^{2} \sigma_{3} f, f\right\rangle .
\end{aligned}
$$

The coefficients in (9.32) satisfy (8.12).

Proof. The proof follows by the tables (9.30), by the product rule for the derivative and by the symmetry properties of $\mathcal{H}$.

We split the proof of Theorem 9.1 in two stages. We first prove step $r=2$ of Theorem 9.1. We subsequently prove the case $r>2$.

\subsection{Proof of Theorem 9.1: the step $r=2$}

At this step, our goal is to obtain a hamiltonian similar to $H$, but with $\widetilde{\mathcal{R}^{(1)}}=0$. We will need to solve a nonlinear homological equation. We consider a $\chi$ like in (9.6) with $M_{0}=1$ satisfying (9.7). We write

$$
H \circ \phi=d\left(\omega_{0}\right)-\omega_{0}\left\|u_{0}\right\|_{2}^{2}+\psi\left(\left\|f^{\prime}\right\|_{2}^{2}\right)+\left(H_{2}^{(1)}+\widetilde{\mathcal{R}^{(1)}}+\widetilde{\mathcal{R}^{(2)}}\right) \circ \phi,
$$

for $\phi$ the Lie transform of $\chi$. We write (9.9)-(9.10) as follows, where we sum on repeated indexes and $\nabla_{f}$ does not act on $\|f\|_{2}^{2}$ :

$$
\begin{gathered}
z_{j}^{\prime}-z_{j}=\partial_{k} \Gamma_{j}\left(0,0,\|f\|_{2}^{2}\right) z_{k}+\partial_{\bar{k}} \Gamma_{j}\left(0,0,\|f\|_{2}^{2}\right) \bar{z}_{k}+ \\
+\left\langle\nabla_{f} \Gamma_{j}\left(0,0,\|f\|_{2}^{2}\right), f\right\rangle+r_{j} \\
f^{\prime}-e^{\mathrm{i} \Gamma_{0}\left(z, f,\|f\|_{2}^{2}\right) P_{c}\left(\omega_{0}\right) \sigma_{3}} f=\partial_{k} \mathcal{G}\left(0,0,\|f\|_{2}^{2}\right) z_{k}+\partial_{\bar{k}} \mathcal{G}\left(0,0,\|f\|_{2}^{2}\right) \bar{z}_{k}+r_{f} .
\end{gathered}
$$

By (9.13)-(??) the terms in $\operatorname{rhs}(9.36)$ satisfy (see (9.8) for definition of $\|\chi\|$ )

$$
\begin{aligned}
& \left|\partial_{k} \Gamma_{j}\right|+\cdots\left\|\partial_{\bar{k}} \mathcal{G}\right\|_{H^{K, S}} \leq C\|\chi\| \\
& \left|r_{j}\right|+\left\|r_{f}\right\|_{H^{K, S}} \leq C\left(|z|^{2}+\|f\|_{H^{-K^{\prime},-S^{\prime}}}^{2}\right) .
\end{aligned}
$$

We write the $f^{\prime \otimes 2}$ in (8.10) schematically as

$$
\begin{aligned}
& f^{\prime 2}(x)=\sum_{|\mu+\nu|=2} A_{\mu \nu}\left(x,\|f\|_{2}^{2}\right) z^{\mu} \bar{z}^{\nu}+\sum_{|\mu+\nu|=1} z^{\mu} \bar{z}^{\nu} \mathcal{A}_{\mu \nu}\left(\|f\|_{2}^{2}\right)(x) f(x) \\
& +\left(e^{\mathrm{i} \Gamma_{0} \sigma_{3}} f+T\left(\Gamma_{0}\right) f\right)^{2}(x)+\varphi(x) r_{j} f(x)+r_{f}(x) f(x)+\varphi(x) r_{j}^{2}+r_{f}^{2}(x)
\end{aligned}
$$


where $\varphi(x)$ represents an exponentially decreasing smooth function. (9.37) implies

$$
\sum_{\mu, \nu}\left\|A_{\mu \nu}\left(x,\|f\|_{2}^{2}\right)\right\|_{H^{K, S}}+\sum_{\mu, \nu}\left\|\mathcal{A}_{\mu \nu}\left(\|f\|_{2}^{2}\right)\right\|_{H^{K, S}} \leq C\|\chi\| .
$$

We consider

$$
\begin{aligned}
& H_{2}^{(1)} \circ \phi+\widetilde{\mathcal{R}^{(1)}} \circ \phi+\int_{\mathbb{R}^{3}} F_{2}\left(x, 0,0,0,\|f\|_{2}^{2}\right) f^{\prime \otimes 2}(x) d x \\
& +\left\langle\nabla_{f}^{2} \widehat{\mathcal{R}}_{2}^{(1)}\left(0,0,\|f\|_{2}^{2}\right), f^{\prime \otimes 2}\right\rangle .
\end{aligned}
$$

We will assume for the moment Lemma 9.5:

Lemma 9.5. The following difference is formed by terms which satisfy the properties stated for $\mathcal{R}^{(2)}$ in Theorem 9.1:

$$
\psi\left(\left\|f^{\prime}\right\|_{2}^{2}\right)+\left(H_{2}^{(1)}+\widetilde{\mathcal{R}^{(1)}}+\widetilde{\mathcal{R}^{(2)}}\right) \circ \phi-\psi\left(\|f\|_{2}^{2}\right)-(9.40) .
$$

We postpone the proof of Lemma 9.5 and focus on (9.40) and on the choice of $\chi$.

Lemma 9.6. It is possible to choose $\chi$ such that there exists $H_{2}^{(2)}$ as in (i) Theorem 9.1 such that the difference $(9.40)-H_{2}^{(2)}$ is formed by terms which satisfy the properties stated for $\mathcal{R}^{(2)}$ in Theorem 9.1.

Proof. We have by (9.6) and using Definition 5.2

$$
\begin{aligned}
& H_{2}^{(1)} \circ \phi=H_{2}^{(1)}+\int_{0}^{1}\left\{H_{2}^{(1)}, \chi\right\} \circ \phi_{t} d t=H_{2}^{(1)}+ \\
& \sum_{|\mu+\nu|=2} b_{\mu \nu}\left(\|f\|_{2}^{2}\right) \int_{0}^{1}\left\{H_{2}^{(1)}, z^{\mu} \bar{z}^{\nu}\right\} \circ \phi_{t} d t+ \\
& \sum_{|\mu+\nu|=1}\left\langle\sigma_{3} \sigma_{1} B_{\mu \nu}\left(\|f\|_{2}^{2}\right), \int_{0}^{1}\left\{H_{2}^{(1)}, z^{\mu} \bar{z}^{\nu} f\right\} \circ \phi_{t} d t\right\rangle+\widetilde{R}
\end{aligned}
$$

with $|\widetilde{R}| \leq C\left(|z|+\|f\|_{H^{-K^{\prime},-S^{\prime}}}\right)^{3}$, (9.30), Lemma 9.2. Then, by (9.30) for $\lambda=\lambda^{(1)}$, defined in (9.24), and substituting $H_{2}^{(1)}=D_{2}^{(1)}+\left(H_{2}^{(1)}-D_{2}^{(1)}\right)$ in the last two lines of (9.42), we get

$$
\begin{aligned}
& H_{2}^{(1)} \circ \phi=H_{2}^{(1)}+\mathrm{i} \sum_{|\mu+\nu|=2} b_{\mu \nu} \lambda \cdot(\mu-\nu) z^{\mu} \bar{z}^{\nu} \\
& -\mathrm{i} \sum_{|\mu+\nu|=1} z^{\mu} \bar{z}^{\nu}\left\langle f, \sigma_{1} \sigma_{3}(\mathcal{H}-\lambda \cdot(\mu-\nu)) B_{\mu \nu}\right\rangle+\widehat{R},
\end{aligned}
$$

with $D_{2}^{(1)}$ defined in (9.28) and with, by (9.30), (8.11) and Lemma 9.2,

$$
|\widehat{R}| \leq C\left(|z|+\|f\|_{H^{-K^{\prime},-S^{\prime}}}\right)^{3}+C\|\chi\|\left(\|\chi\|+\|f\|_{2}^{2}\right)\left(|z|+\|f\|_{H^{-K^{\prime},-S^{\prime}}}\right)^{2} .
$$


Similarly

$$
\begin{gathered}
\widetilde{\mathcal{R}^{(1)}} \circ \phi=\widetilde{\mathcal{R}^{(1)}}+\sum_{|\mu+\nu|=2} \widetilde{l}_{\mu \nu} z^{\mu} \bar{z}^{\nu}+\sum_{|\mu+\nu|=1} z^{\mu} \bar{z}^{\nu}\left\langle f, \sigma_{1} \sigma_{3} \widetilde{L}_{\mu \nu}\right\rangle+\underline{R}, \\
\text { with } \widetilde{l}_{\mu \nu} \mid+\left\|\widetilde{L}_{\mu \nu}\right\|_{H^{K, S}} \leq C\|\chi\|\left\|\widetilde{\mathcal{R}^{(1)}}\right\| \\
|\underline{R}| \leq \operatorname{rhs}(9.43)+\operatorname{rhs}(9.45) .
\end{gathered}
$$

In (9.40) we substitute $f^{\prime \otimes 2}$ using (9.38). Then

$$
\int_{\mathbb{R}^{3}} F_{2}\left(x, 0,0,0,\|f\|_{2}^{2}\right) f^{\prime \otimes 2}(x) d x=\tilde{\chi}+\mathrm{R}
$$

with: $\tilde{\chi}$ a polynomial like (9.6) with $M_{0}=1$ such that $\|\widetilde{\chi}\| \leq C\|f\|_{2}^{2}\|\chi\|$ by claims (4) and (9) in Lemma 8.3 and by (9.38); $\widetilde{\chi}$ satisfies (9.7) by the fact that the $\operatorname{rhs}(9.47)$ is real valued; $\mathrm{R}$ formed by terms with the properties stated for $\mathcal{R}^{(2)}$ in Theorem 9.1, see second line of (9.38). By an argument similar to the one for (9.47), we have

$$
\left\langle\nabla_{f}^{2} \widehat{\mathcal{R}}_{2}^{(1)}\left(0,0,\|f\|_{2}^{2}\right), f^{\prime \otimes 2}\right\rangle=\tilde{\chi}+\mathrm{R},
$$

with $\tilde{\chi}$ and $\mathrm{R}$ different from the ones in (9.47) but with the same properties. Then we have

$$
\begin{aligned}
& (9.40)=H_{2}^{(1)}+\widetilde{\mathcal{R}}^{(2)}+\widehat{\chi}+\mathrm{i} \sum_{|\mu+\nu|=2} b_{\mu \nu} \lambda \cdot(\mu-\nu) z^{\mu} \bar{z}^{\nu}- \\
& -\mathrm{i} \sum_{|\mu+\nu|=1} z^{\mu} \bar{z}^{\nu}\left\langle f, \sigma_{1} \sigma_{3}(\mathcal{H}-\lambda \cdot(\mu-\nu)) B_{\mu \nu}\right\rangle+\mathbf{R}
\end{aligned}
$$

where $\mathbf{R}$ satisfies the properties stated for $\mathcal{R}^{(2)}$ and $\widehat{\chi}$ is a polynomial like (9.6)(9.7) with $M_{0}=1$ and ( $\widehat{Z}$ and $\widehat{K}$ will be defined in two lines)

$$
\|\widehat{\chi}\|=\|\widehat{Z}\|+\|\widehat{K}\| \leq C\|\chi\|\left(\|f\|_{2}^{2}+\|\chi\|+\left\|\widetilde{\mathcal{R}}^{(2)}\right\|\right) .
$$

Here $\widehat{\chi}=\widehat{Z}+\widehat{K}$, where in $\widehat{Z}=\sum \widehat{b}_{\mu \nu}\left(\|f\|_{2}^{2}\right) z^{\mu} \bar{z}^{\nu}$ we sum over $|\mu+\nu|=2$, $\lambda^{0} \cdot \mu=\lambda^{0} \cdot \nu$, i.e. in $\widehat{Z}$ we collect the null form terms of $\widehat{\chi}$. We set

$$
H_{2}^{(2)}=H_{2}^{(1)}+\widehat{Z} .
$$

Up to now $\chi$ is undetermined. We choose $\chi$ with coefficients $b_{\mu \nu}$ and $B_{\mu \nu}$ such that $b_{\mu \nu}=0$ for $\lambda^{0} \cdot \mu=\lambda^{0} \cdot \nu$ and such that the following system is satisfied:

$$
\begin{aligned}
& \widetilde{\mathcal{R}^{(1)}}+\widehat{K}+\mathrm{i} \sum_{|\mu+\nu|=2} b_{\mu \nu} \lambda \cdot(\mu-\nu) z^{\mu} \bar{z}^{\nu}- \\
& -\mathrm{i} \sum_{|\mu+\nu|=1} z^{\mu} \bar{z}^{\nu}\left\langle f, \sigma_{1} \sigma_{3}(\mathcal{H}-\lambda \cdot(\mu-\nu)) B_{\mu \nu}\right\rangle=0 .
\end{aligned}
$$


In coordinates, $(9.52)$ is

$$
\begin{aligned}
& a_{\mu \nu}^{(1)}+\widehat{k}_{\mu \nu}+\mathrm{i} b_{\mu \nu} \lambda \cdot(\mu-\nu)=0,|\mu+\nu|=2, \lambda^{0} \cdot \mu \neq \lambda^{0} \cdot \nu, \\
& G_{\mu \nu}+\widehat{K}_{\mu \nu}-\mathrm{i}(\mathcal{H}-\lambda \cdot(\mu-\nu)) B_{\mu \nu}=0,|\mu+\nu|=1
\end{aligned}
$$

where: $a_{\mu \nu}^{(1)}$ and $G_{\mu \nu}$ are coefficients of $\widetilde{\mathcal{R}^{(1)}}$, they are smooth functions of $\varrho=$ $\|f\|_{2}^{2}$, and are equal to 0 for $\varrho=0 ; \widehat{k}_{\mu \nu} \in \mathbb{C}$ and $\widehat{K}_{\mu \nu} \in H^{K, S}$ are coefficients of $\widehat{K}$, and are smooth functions of $\varrho=\|f\|_{2}^{2}$ and of the coefficients of $\chi$, where $b_{\mu \nu} \in \mathbb{C}$ and $B_{\mu \nu} \in H^{K, S}$. By $(9.50)$

$$
\left|\widehat{k}_{\mu \nu}\right|+\left\|\widehat{K}_{\mu \nu}\right\|_{H^{K, S}} \leq C\|\chi\|\left(\|f\|_{2}^{2}+\|\chi\|+\left\|\widetilde{\mathcal{R}^{(1)}}\right\|\right) .
$$

Then by the implicit function theorem we can solve the nonlinear system (9.53) with unknown $\chi$ obtaining (we consider $b_{\mu \nu}$ only for $\lambda^{0} \cdot \mu \neq \lambda^{0} \cdot \nu$ )

$$
\begin{aligned}
& \left|b_{\mu \nu}+\frac{a_{\mu \nu}^{(1)}}{\mathrm{i} \lambda \cdot(\mu-\nu)}\right|+\left\|B_{\mu \nu}+\mathrm{i} R_{\mathcal{H}}(\lambda \cdot(\mu-\nu)) G_{\mu \nu}\right\|_{H^{K, S}} \\
& \leq C\left\|\widetilde{\mathcal{R}^{(1)}}\right\|\left(\left\|\widetilde{\mathcal{R}^{(1)}}\right\|+\|f\|_{2}^{2}\right) .
\end{aligned}
$$

Notice that with the above choice of $\chi$ and with (9.51), (9.49) yields

$$
(9.40)=H_{2}^{(2)}+\mathbf{R},
$$

where $\mathbf{R}$ has the properties stated for $\mathcal{R}^{(2)}$ in Theorem 9.1. Hence Lemma 9.5 is proved.

\section{Proof of Lemma 9.5}

By (9.11)-(9.12), and with the big O smooth in $z \in \mathbb{C}^{m}, f \in H_{c}^{-K^{\prime},-S^{\prime}}$,

$$
\psi\left(\left\|f^{\prime}\right\|_{2}^{2}\right)=\psi\left(\|f\|_{2}^{2}\right)+O\left(|z|^{2}\|f\|_{H^{-K^{\prime},-S^{\prime}}}+\|f\|_{H^{-K^{\prime},-S^{\prime}}}^{3}\right) .
$$

The error term in (9.57) has the properties stated for $\mathcal{R}^{(2)}$ in Theorem 9.1. We consider the terms $\widetilde{\mathcal{R}^{(2)}} \circ \phi$. Terms, for $|\mu+\nu|=3$, like

$$
z^{\prime \mu} \bar{z}^{\prime \nu} \int_{\mathbb{R}^{3}} a\left(x, z^{\prime}, f^{\prime}, f^{\prime}(x),\left\|f^{\prime}\right\|_{2}^{2}\right) d x
$$

by (9.9) and (9.13) can be written as

$$
\left(z^{\mu} \bar{z}^{\nu}+O\left(\left(|z|+\|f\|_{H^{-K^{\prime},-S^{\prime}}}\right)^{3}\right)\right) \int_{\mathbb{R}^{3}} a\left(x, z^{\prime}, f^{\prime}, f^{\prime}(x),\left\|f^{\prime}\right\|_{2}^{2}\right) d x,
$$

In the notation of Lemma 9.2 we have

$$
\begin{aligned}
& a\left(x, z^{\prime}, f^{\prime}, f^{\prime}(x),\left\|f^{\prime}\right\|_{2}^{2}\right)=a\left(x, z+\Gamma, e^{\mathrm{i} \Gamma_{0} P_{c}\left(\omega_{0}\right) \sigma_{3}} f+\mathcal{G},\right. \\
& \left.e^{\mathrm{i} \Gamma_{0} \sigma_{3}} f(x)+\left[T\left(\Gamma_{0}\right) f\right](x)+\mathcal{G}\left(z, f,\|f\|_{2}^{2}\right)(x),\|f\|_{2}^{2}+\Gamma_{1}\right) \\
& =a\left(x, z, f, f(x),\|f\|_{2}^{2}\right)+O\left(|z|+\|f\|_{H^{-K^{\prime},-S^{\prime}}}\right) .
\end{aligned}
$$


The big O's in (9.59)-(9.60) are smooth in $z \in \mathbb{C}^{m}, f \in H_{c}^{-K^{\prime},-S^{\prime}}$. Then (9.58) has the properties stated for $\mathcal{R}^{(2)}$ in Theorem 9.1. Similar formulas can be used for

$$
\begin{aligned}
& \sum_{|\mu+\nu|=2} z^{\prime \mu} \bar{z}^{\prime \nu} \int_{\mathbb{R}^{3}}\left[\sigma_{1} \sigma_{3} G_{\mu \nu}\left(x, z^{\prime}, f^{\prime}, f^{\prime}(x),\left\|f^{\prime}\right\|_{2}^{2}\right)\right]^{*} f^{\prime}(x) d x+ \\
& \sum_{j=3}^{5} \int_{\mathbb{R}^{3}} F_{j}\left(x, z^{\prime}, f^{\prime}, f^{\prime}(x),\left\|f^{\prime}\right\|_{2}^{2}\right) f^{\prime \otimes j}(x) d x+\int_{\mathbb{R}^{3}} B\left(\left|f^{\prime}(x)\right|^{2} / 2\right) d x .
\end{aligned}
$$

We treat with some detail these terms in the step $r>2$, Subsection 9.4. Next we consider the term with $\int F_{2} f^{\prime \otimes 2}(x) d x$. First of all, we can apply to $F_{2}$ an analogue of (9.60) to obtain for $d=2$

$$
\begin{aligned}
& F_{d}\left(x, z^{\prime}, f^{\prime}, f^{\prime}(x),\left\|f^{\prime}\right\|_{2}^{2}\right)=F_{d}\left(x, z+\Gamma, e^{\mathrm{i} \Gamma_{0} P_{c}\left(\omega_{0}\right) \sigma_{3}} f+\mathcal{G},\right. \\
& \left.e^{\mathrm{i} \Gamma_{0} \sigma_{3}} f(x)+\left[T\left(\Gamma_{0}\right) f\right](x)+\mathcal{G}\left(z, f,\|f\|_{2}^{2}\right)(x),\|f\|_{2}^{2}+\Gamma_{1}\right) \\
& =F_{d}\left(x, 0,0, f(x),\|f\|_{2}^{2}\right)+O\left(|z|+\|f\|_{H^{-K^{\prime}},-S^{\prime}}\right) .
\end{aligned}
$$

Then, modulo terms with the properties stated for $\mathcal{R}^{(2)}$ in Theorem 9.1, we get

$$
\begin{aligned}
& \int_{\mathbb{R}^{3}} F_{2}\left(x, 0,0, f(x),\|f\|_{2}^{2}\right) f^{\prime \otimes 2}(x) d x=\int_{\mathbb{R}^{3}} F_{2}\left(x, 0,0,0,\|f\|_{2}^{2}\right) f^{\prime \otimes 2}(x) d x \\
& +\int_{\mathbb{R}^{3}} G_{2}\left(x, f(x),\|f\|_{2}^{2}\right) f(x) \otimes f^{\prime \otimes 2}(x) d x
\end{aligned}
$$

where first term in rhs has been treated in Lemma 9.6 and second term has the properties stated for $\mathcal{R}_{3}^{(2)}$ in Theorem 9.1. By a similar argument

$$
\widehat{\mathcal{R}}_{2}^{(1)}\left(z^{\prime}, f^{\prime},\left\|f^{\prime}\right\|_{2}\right)-\left\langle\nabla_{f}^{2} \widehat{\mathcal{R}}_{2}^{(1)}\left(0,0,\|f\|_{2}^{2}\right), f^{\prime \otimes 2}\right\rangle
$$

has the properties stated for $\mathcal{R}^{(2)}$ in Theorem 9.1.

We denote: $\chi_{2}=\chi, \mathcal{T}_{2}$ the Lie transformation of $\chi_{2}, Z^{(2)}=0 . H_{2}^{(2)}$ has been defined in (9.51). We denote $\mathcal{R}^{(2)}=(9.41)+(9.40)-H_{2}^{(2)}$. This $\mathcal{R}^{(2)}$ satisfies the conditions in Theorem 9.1. This ends the proof of case $r=2$ in Theorem 9.1.

\subsection{Proof of Theorem 9.1: the step $r>2$}

Case $r=2$ has been treated in Subsection 9.3. We have defined $H_{2}^{(2)}$ in (9.51). We proceed by induction to complete the proof of Theorem 9.1. From the argument below one can see that $H_{2}^{(r)}=H_{2}^{(2)}$ for all $r \geq 2$. For $r \geq 2$, write Taylor expansions

$$
\mathcal{R}_{0}^{(r)}-\mathcal{R}_{02}^{(r)}=\sum_{|\mu+\nu|=r+1} z^{\mu} \bar{z}^{\nu} \int_{\mathbb{R}^{3}} a_{\mu \nu}^{(r)}\left(x, 0,0,0,\|f\|_{2}^{2}\right) d x
$$




$$
\mathcal{R}_{1}^{(r)}-\mathcal{R}_{12}^{(r)}=\sum_{|\mu+\nu|=r} z^{\mu} \bar{z}^{\nu} \int_{\mathbb{R}^{3}}\left[\sigma_{1} \sigma_{3} G_{\mu \nu}^{(r)}\left(x, 0,0,0,\|f\|_{2}^{2}\right)\right]^{*} f(x) d x .
$$

We have

$$
\begin{gathered}
\mathcal{R}_{02}^{(r)}+\mathcal{R}_{12}^{(r)}=\sum_{|\mu+\nu|=r+2} z^{\mu} \bar{z}^{\nu} \int_{\mathbb{R}^{3}} \widetilde{a}_{\mu \nu}^{(r)}\left(x, z, f, 0,\|f\|_{2}^{2}\right) d x+ \\
\sum_{|\mu+\nu|=r+1} z^{\mu} \bar{z}^{\nu} \int_{\mathbb{R}^{3}}\left[\sigma_{1} \sigma_{3} \widetilde{G}_{\mu \nu}^{(r)}\left(x, z, f, f(x),\|f\|_{2}^{2}\right)\right]^{*} f(x) d x+ \\
\sum_{|\mu+\nu|=r} z^{\mu} \bar{z}^{\nu} \int_{\mathbb{R}^{3}} \widetilde{F}_{2}^{(r)}\left(x, z, f, f(x),\|f\|_{2}^{2}\right) \cdot(f(x))^{\otimes 2} d x
\end{gathered}
$$

with $\widetilde{a}_{\mu \nu}^{(r)}$ satisfying $(9.2), \widetilde{G}_{\mu \nu}^{(r)}(9.3)$ and $\widetilde{F}_{2}^{(r)}(9.4)$. Since $H^{(r)}=H \circ \mathcal{T}_{r}$ is real valued (because $H$ is real valued), then both sides of equations (9.65)-(9.67) are real valued. In particular, $a_{\mu \nu}^{(r)}$ and $G_{\mu \nu}^{(r)}$ satisfy (8.12). Set

$$
\widetilde{K}_{r+1}:=\operatorname{rhs}(9.65)+\operatorname{rhs}(9.66) \text {. }
$$

Split $\widetilde{K}_{r+1}=K_{r+1}+Z_{r+1}$ collecting inside $Z_{r+1}$ all the terms of $\widetilde{K}_{r+1}$ in null form. The coefficients of $K_{r+1}$ and of $Z_{r+1}$ satisfy (8.12), by the argument just before (9.68). We consider a (momentarily unknown) polynomial $\chi$ like (9.6)(9.7), $M_{0}=r$. Denote by $\phi$ its Lie transformation. Let $\left(z^{\prime}, f^{\prime}\right)=\phi(z, f)$. For $d=2$, in the notation of Lemma 9.2 we have

$$
\left(\mathcal{R}_{d}^{(r)}-\widehat{\mathcal{R}}_{d}^{(r)}\right)\left(z^{\prime}, f^{\prime}\right)=\left\langle F_{d}^{(r)}\left(z^{\prime}, f^{\prime}, f^{\prime}(\cdot),\left\|f^{\prime}\right\|_{2}^{2}\right),\left(e^{\mathrm{i} \Gamma_{0} P_{c}\left(\omega_{0}\right) \sigma_{3}} f+\mathcal{G}\right)^{\otimes d}\right\rangle
$$

Then $\operatorname{rhs}(9.69)=$

$$
\begin{aligned}
& =\sum_{j=0}^{d}\left(\begin{array}{l}
d \\
j
\end{array}\right)\left\langle F_{d}^{(r)}\left(z^{\prime}, f^{\prime}, f^{\prime}(\cdot),\left\|f^{\prime}\right\|_{2}^{2}\right), \mathcal{G}^{\otimes(d-j)} \otimes\left[e^{\mathrm{i} \Gamma_{0} P_{c}\left(\omega_{0}\right) \sigma_{3}} f\right]^{\otimes j}\right\rangle= \\
& \sum_{j=0}^{d}\left(\begin{array}{l}
d \\
j
\end{array}\right) \sum_{\ell=0}^{j}\left(\begin{array}{l}
j \\
\ell
\end{array}\right)\left\langle F_{d}^{(r)}(\cdots), \mathcal{G}^{\otimes(d-j)} \otimes\left[T\left(\Gamma_{0}\right) f\right]^{\otimes(j-\ell)} \otimes\left[e^{\mathrm{i} \Gamma_{0} \sigma_{3}} f\right]^{\otimes \ell}\right\rangle .
\end{aligned}
$$

In the notation of Lemma 9.2 we have for $d=2$

$$
\begin{aligned}
& F_{d}^{(r)}\left(z^{\prime}, f^{\prime}, f^{\prime}(x),\left\|f^{\prime}\right\|_{2}^{2}\right)(x)= \\
& F_{d}^{(r)}\left(z+\Gamma, e^{\mathrm{i} \Gamma_{0} P_{c}\left(\omega_{0}\right) \sigma_{3}} f+\mathcal{G}, e^{\mathrm{i} \Gamma_{0} \sigma_{3}} f(x)+\left[T\left(\Gamma_{0}\right) f\right](x),\|f\|_{2}^{2}+\Gamma_{1}\right)(x) .
\end{aligned}
$$

Then

$$
\begin{aligned}
& F_{2}^{(r)}\left(z^{\prime}, f^{\prime}, f^{\prime}(x),\left\|f^{\prime}\right\|_{2}^{2}\right)(x)=F_{2}^{(r)}\left(0,0, f(x),\|f\|_{2}^{2}\right)(x)+ \\
& O\left(|z|+\|f\|_{H^{-K^{\prime},-S^{\prime}}}\right)=F_{2}^{(r)}\left(0,0,0,\|f\|_{2}^{2}\right)(x)+ \\
& G\left(0,0, f(x),\|f\|_{2}^{2}\right)(x) f(x)+O\left(|z|+\|f\|_{H^{-K^{\prime},-S^{\prime}}}\right),
\end{aligned}
$$


where the big $\mathrm{O}$ are smooth in $z \in \mathbb{C}^{m}$ and $f \in H^{-K^{\prime},-S^{\prime}}$ with values in $H^{K, S}\left(\mathbb{R}^{3}, B\left(\left(\mathbb{C}^{2}\right)^{\otimes 2}, \mathbb{C}\right)\right.$ and where $G$ has values in $H^{K, S}\left(\mathbb{R}^{3}, B\left(\left(\mathbb{C}^{2}\right)^{\otimes 3}, \mathbb{C}\right)\right.$ and satisfies estimates (9.4). So the last line of (9.72) when plugged in (9.70) for $d=2$ yields terms with the properties of $\sum_{d=0}^{3} \mathcal{R}_{d}^{(r+1)}$. We focus now on the first term in the rhs of (9.72). Schematically, in analogy to (9.38) we write

$$
\begin{aligned}
& f^{\prime 2}(x)=\sum_{|\mu+\nu|=r} z^{\mu} \bar{z}^{\nu} \mathcal{A}_{\mu \nu}\left(\|f\|_{2}^{2}\right)(x) f(x) \\
& +\sum_{|\mu+\nu|=2 r} A_{\mu \nu}\left(x,\|f\|_{2}^{2}\right) z^{\mu} \bar{z}^{\nu}+\left(e^{\mathrm{i} \Gamma_{0} \sigma_{3}} f+T\left(\Gamma_{0}\right) f\right)^{2}(x) \\
& +\varphi(x) r_{j} f(x)+r_{f}(x) f(x)+\varphi(x) r_{j}^{2}+r_{f}^{2}(x),
\end{aligned}
$$

where we have (9.39) and $\left|r_{j}\right|+\left\|r_{f}\right\|_{H^{K, S}} \leq C\left(|z|+\|f\|_{H^{-K^{\prime},-S^{\prime}}}\right)^{r+1}$. Then

$$
\int_{\mathbb{R}^{3}} F_{2}^{(r)}\left(x, 0,0,0,\|f\|_{2}^{2}\right) f^{\prime \otimes 2}(x) d x=\tilde{\chi}_{1}+\mathrm{R}_{1}
$$

with: $R_{1}$ formed by terms obtained by the last two lines of $(9.73)$ has the properties stated for $\mathcal{R}^{(r+1)}$ in Theorem $9.1 ; \widetilde{\chi}_{1}$ a polynomial like (9.6) with $M_{0}=r$ arising from the first line of rhs of (9.73) is such that $\left\|\widetilde{\chi}_{1}\right\| \leq C\|f\|_{2}^{2}\|\chi\|$ by the inductive hypothesis $F_{2}^{(r)}(x, 0,0,0,0)=0$ in (iv.2-5) Theorem 9.1 and by (9.39); $\widetilde{\chi}_{1}$ satisfies (9.7) because each side in (9.74) is real valued. We have

$$
\begin{aligned}
& \widehat{\mathcal{R}}_{2}^{(r)}\left(z^{\prime}, f^{\prime},\left\|f^{\prime}\right\|_{2}^{2}\right)=\left\langle\nabla_{f}^{2} \widehat{\mathcal{R}}_{2}^{(1)}\left(0,0,\|f\|_{2}^{2}\right), f^{\prime \otimes 2}\right\rangle+ \\
& +\left(\widehat{\mathcal{R}}_{2}^{(r)}\left(z^{\prime}, f^{\prime},\left\|f^{\prime}\right\|_{2}^{2}\right)-\left\langle\nabla_{f}^{2} \widehat{\mathcal{R}}_{2}^{(r)}\left(0,0,\|f\|_{2}^{2}\right), f^{\prime \otimes 2}\right\rangle\right),
\end{aligned}
$$

where the second line on rhs of (9.75) yields terms which have the properties of elements of $\mathcal{R}^{(r+1)}$. We have

$$
\left\langle\nabla_{f}^{2} \widehat{\mathcal{R}}_{2}^{(r)}\left(0,0,\|f\|_{2}^{2}\right), f^{\prime \otimes 2}\right\rangle=\widetilde{\chi}_{2}+\mathrm{R}_{2}
$$

where $\widetilde{\chi}_{2}$ and $\mathrm{R}_{2}$ have the same properties of $\widetilde{\chi}_{1}$ and $\mathrm{R}_{1}$ in (9.76). Split $H_{2}^{(r)}=$ $D_{2}^{(r)}+\left(H_{2}^{(r)}-D_{2}^{(r)}\right)$ for $D_{2}^{(r)}$ in (9.28). Then

$$
\left\{\chi, H_{2}^{(r)}-D_{2}^{(r)}\right\}=\widetilde{\chi}_{3}+\mathrm{R}_{3}
$$

where $\widetilde{\chi}_{3}$ and $R_{3}$ have the same properties of $\widetilde{\chi}_{1}$ and $R_{1}$ in (9.76). Set $\tilde{\chi}=$ $\sum_{j=1}^{3} \tilde{\chi}_{j}$. Split now $\widetilde{\chi}=\widetilde{Z}+\widehat{K}$ collecting in $\widetilde{Z}$ the null form terms in $\widetilde{\chi}$. Then we choose the yet unknown $\chi$ such that its coefficients $b_{\mu \nu}$ and $B_{\mu \nu}$ satisfy the system

$$
\begin{aligned}
& \widetilde{K}_{r+1}+\widehat{K}+\mathrm{i} \sum_{|\mu+\nu|=2} b_{\mu \nu} \lambda \cdot(\mu-\nu) z^{\mu} \bar{z}^{\nu}- \\
& -\mathrm{i} \sum_{|\mu+\nu|=1} z^{\mu} \bar{z}^{\nu}\left\langle f, \sigma_{1} \sigma_{3}(\mathcal{H}-\lambda \cdot(\mu-\nu)) B_{\mu \nu}\right\rangle=0 .
\end{aligned}
$$


Notice that for $\widehat{K} \equiv 0$ system (9.78) would be linear and admit exactly one solution. By $\|\widetilde{\chi}\| \leq C\|f\|_{2}^{2}\|\chi\|$ we get $\|\widehat{K}\| \leq C\|f\|_{2}^{2}\|\chi\|$. So by the implicit function theorem there exists exactly one solution of (9.78). This solution is close to the solution of system (9.78) when $\widehat{K} \equiv 0$. Furthermore, this system has solution $\chi_{r+1}=\chi$ which satisfies (8.12), or what is the same, (9.7). For $L_{r+1}$ of type (9.34), $\chi_{r+1}$ satisfies

$$
\left\{\chi_{r+1}, H_{2}^{(r)}\right\}=\widetilde{K}_{r+1}+\widehat{K}+L_{r+1} .
$$

Call $\phi_{r+1}=\phi$ the Lie transform of $\chi_{r+1}$. For $\mathcal{T}_{r+1}=\mathcal{T}_{r} \circ \phi_{r+1}$ set

$$
H^{(r+1)}:=H^{(r)} \circ \phi_{r+1}=H \circ\left(\mathcal{T}_{r} \circ \phi_{r+1}\right)=H \circ \mathcal{T}_{r+1} .
$$

Since $\chi_{r+1}$ satisfies $(8.12), H^{(r+1)}$ is well defined and real valued. Split

$$
\begin{gathered}
H^{(r)} \circ \phi_{r+1}=H_{2}^{(r)}+Z^{(r)}+Z_{r+1}+\widetilde{Z} \\
+\left(Z^{(r)} \circ \phi_{r+1}-Z^{(r)}\right)+\left(\widetilde{Z} \circ \phi_{r+1}-\widetilde{Z}\right) \\
+\left(\widetilde{K}_{r+1}+\widehat{K}\right) \circ \phi_{r+1}-\widetilde{K}_{r+1}-\widehat{K} \\
+H_{2}^{(r)} \circ \phi_{r+1}-\left(H_{2}^{(r)}+\left\{H_{2}^{(r)}, \chi_{r+1}\right\}\right) \\
\quad+\left(\mathcal{R}_{02}^{(r)}+\mathcal{R}_{12}^{(r)}\right) \circ \phi_{r+1} \\
+\sum_{d=3}^{5}\left(\mathcal{R}_{d}^{(r)}-\widehat{\mathcal{R}}_{d}^{(r)}\right) \circ \phi_{r+1}+\widehat{\mathcal{R}}_{d}^{(r)} \circ \phi_{r+1} \\
+\left(\mathcal{R}_{2}^{(r)}-\widetilde{Z}-\widehat{K}\right) \circ \phi_{r+1} \\
+\psi \circ \phi_{r+1}+\mathcal{R}_{6}^{(r)} \circ \phi_{r+1} .
\end{gathered}
$$

Define $H_{2}^{(r+1)}=H_{2}^{(r)}$ (this proves $H_{2}^{(r)}=H_{2}^{(2)}$ ) and $Z^{(r+1)}:=Z^{(r)}+Z_{r+1}+\widetilde{Z}$. Its coefficients satisfy (8.12) (because $H^{(r+1)}$ is real valued) and it is a normal form. We have already discussed that (9.87) has the properties stated for $\mathcal{R}^{(r+1)}$. By expansions (9.69)-(9.71) we get that the first summation in (9.86) has the properties stated for $\mathcal{R}^{(r+1)}$. By an analogous argument, terms $\widehat{\mathcal{R}}_{d}^{(r)}\left(z^{\prime}, f^{\prime}\right)$ have the properties stated for $\mathcal{R}^{(r+1)}$. We have, for $T=T\left(\Gamma_{0}\right)$,

$$
\begin{aligned}
& \left|f^{\prime}(x)\right|^{2}=|f(x)|^{2}+\mathcal{E}(x) \text { with } \mathcal{E}(x):=2\left(T\left(\Gamma_{0}\right) f(x)\right)^{*} \sigma_{1} e^{\mathrm{i} \Gamma_{0} \sigma_{3}} f(x) \\
& +\left|T\left(\Gamma_{0}\right) f(x)\right|^{2}+2 \mathcal{G}^{*}(x) \sigma_{1} e^{\mathrm{i} \Gamma_{0} \sigma_{3}} f(x)+2 \mathcal{G}^{*}(x) \sigma_{1} T\left(\Gamma_{0}\right) f(x)+|\mathcal{G}(x)|^{2} .
\end{aligned}
$$

Then

$$
\begin{aligned}
& \mathcal{R}_{6}^{(r)} \circ \phi_{r+1}=\int_{\mathbb{R}^{3}} B\left(\left|f^{\prime}(x)\right|^{2} / 2\right) d x=\int_{\mathbb{R}^{3}} B\left(|f(x)|^{2} / 2\right) d x \\
& +\frac{1}{2} \int_{\mathbb{R}^{3}} d x \mathcal{E}(x) \int_{0}^{1} B^{\prime}\left(|f(x)|^{2} / 2+s \mathcal{E}(x) / 2\right) d s .
\end{aligned}
$$

The last line in (9.90) has the properties stated for $\mathcal{R}^{(r+1)}-\mathcal{R}_{6}^{(r+1)}$ by Lemma 9.2. By (9.67) and by the fact that $\widetilde{a}_{\mu \nu}^{(r)}$ satisfies $(9.2), \widetilde{G}_{\mu \nu}^{(r)}(9.3)$ and $\widetilde{F}_{2}^{(r)}(9.4)$, 
the terms $\mathcal{R}_{02}^{(r)}+\mathcal{R}_{12}^{(r)}$ has the properties stated for $\sum_{d=0}^{2} \mathcal{R}_{d}^{(r+1)}$. The same conclusion holds for (9.85). By Lemma 9.2 and by an analogue of (9.57), we have that $\psi \circ \phi_{r}=\psi+\widetilde{\psi}$ where $\widetilde{\psi}$ has the properties stated for $\sum_{d=1}^{3} \mathcal{R}_{d}^{(r+1)}$ by (9.12). We have

$$
Z^{(r)} \circ \phi_{r+1}-Z^{(r)}=\int_{0}^{1}\left\{Z^{(r)}, \chi_{r+1}\right\} \circ \phi_{r+1}^{t} d t .
$$

We have

$$
\left|\left\{\chi_{r+1}, Z^{(r)}\right\}\right| \leq C\left(|z|^{r+2}+|z|^{r+1}\|f\|_{H^{-K^{\prime},-S^{\prime}}}\right) .
$$

By (9.92) we conclude that (9.91) has the properties stated for $\mathcal{R}^{(r+1)}$. The same is true for the other terms in (9.82)-(9.83). We have, for $H_{2}=H_{2}^{(r)}$,

$$
\begin{aligned}
& H_{2} \circ \phi_{r+1}-\left(H_{2}+\left\{H_{2}, \chi_{r+1}\right\}\right)=\int_{0}^{1} \frac{t^{2}}{2 !}\left\{\left\{H_{2}, \chi_{r+1}\right\}, \chi_{r+1}\right\} \circ \phi_{r+1}^{t} d t \\
& =-\int_{0}^{1} \frac{t^{2}}{2 !}\left\{K_{r+1}+\widehat{K}+L_{r+1}, \chi_{r+1}\right\} \circ \phi_{r+1}^{t} d t .
\end{aligned}
$$

Then $\left|\left\{K_{r+1}+\widehat{K}+L_{r+1}, \chi_{r+1}\right\}\right| \leq$ rhs (9.92) implies that (9.93) has the properties stated for $\mathcal{R}^{(r+1)}$.

\section{Dispersion}

We apply Theorem 9.1 for $r=2 N+1$ (recall $N=N_{1}$ where $N_{j} \lambda_{j}<\omega_{0}<$ $\left.\left(N_{j}+1\right) \lambda_{j}\right)$. In the rest of the paper we work with the hamiltonian $H^{(r)}$. We will drop the upper index. So we will set $H=H^{(r)}, H_{2}=H_{2}^{(r)}, \lambda_{j}=\lambda_{j}^{(r)}$, $\lambda=\lambda^{(r)}, Z_{a}=Z_{a}^{(r)}$ for $a=0,1$ and $\mathcal{R}=\mathcal{R}^{(r)}$. In particular we will denote by $G_{\mu \nu}$ the coefficients $G_{\mu \nu}^{(r)}$ of $Z_{1}^{(r)}$. We will show:

Theorem 10.1. There is a fixed $C>0$ such that for $\varepsilon_{0}>0$ sufficiently small and for $\epsilon \in\left(0, \varepsilon_{0}\right)$ we have

$$
\begin{gathered}
\|f\|_{L_{t}^{r}\left([0, \infty), W_{x}^{1, p}\right)} \leq C \epsilon \text { for all admissible pairs }(r, p) \\
\left\|z^{\mu}\right\|_{L_{t}^{2}([0, \infty))} \leq C \epsilon \text { for all multi indexes } \mu \text { with } \lambda \cdot \mu>\omega_{0} \\
\left\|z_{j}\right\|_{W_{t}^{1, \infty}([0, \infty))} \leq C \epsilon \text { for all } j \in\{1, \ldots, m\} .
\end{gathered}
$$

Estimate (10.3) is a consequence of the classical proof of orbital stability in Weinstein [W1]. Notice that (1.1) is time reversible, so in particular (10.1)(10.3) are true over the whole real line. The proof, though, exploits that $t \geq 0$, specifically when for $\lambda \in \sigma_{c}(\mathcal{H})$ we choose $R_{\mathcal{H}}^{+}(\lambda)=R_{\mathcal{H}}(\lambda+\mathrm{i} 0)$ rather than $R_{\mathcal{H}}^{-}(\lambda)=R_{\mathcal{H}}(\lambda-\mathrm{i} 0)$ in formula (10.11). See the discussion on p.18 [SW3]. 
The proof of Theorem 10.1 involves a standard continuation argument. We assume

$$
\begin{gathered}
\|f\|_{L_{t}^{r}\left([0, T], W_{x}^{1, p}\right)} \leq C_{1} \epsilon \text { for all admissible pairs }(r, p) \\
\left\|z^{\mu}\right\|_{L_{t}^{2}([0, T])} \leq C_{2} \epsilon \text { for all multi indexes } \mu \text { with } \omega \cdot \mu>\omega_{0}
\end{gathered}
$$

for fixed sufficiently large constants $C_{1}, C_{2}$ and then we prove that for $\epsilon$ sufficiently small, (10.4) and (10.5) imply the same estimate but with $C_{1}, C_{2}$ replaced by $C_{1} / 2, C_{2} / 2$. Then $(10.4)$ and $(10.5)$ hold with $[0, T]$ replaced by $[0, \infty)$.

The proof consists in three main steps.

(i) Estimate $f$ in terms of $z$.

(ii) Substitute the variable $f$ with a new "smaller" variable $g$ and find smoothing estimates for $g$.

(iii) Reduce the system for $z$ to a closed system involving only the $z$ variables, by insulating the part of $f$ which interacts with $z$, and by decoupling the rest (this reminder is $g$ ). Then clarify the nonlinear Fermi golden rule.

The first two steps are the same of $[\mathrm{CM}]$. The only novelty of the proof with respect to $[\mathrm{CM}]$ is step (iii), specifically the part on the Fermi golden rule. At issue is the non negativity of some crucial coefficients in the equations of $z$. This point is solved using the same ideas in Lemma $5.2[\mathrm{BC}]$. The fact that they are not 0 is assumed by hypothesis (H11). The fact that if not 0 they are positive, is proved here.

Step (i) is encapsulated by the following proposition:

Proposition 10.2. Assume (10.4) and (10.5). Then there exist constants $C=C\left(C_{1}, C_{2}\right), K_{1}$, with $K_{1}$ independent of $C_{1}$, such that, if $C\left(C_{1}, C_{2}\right) \epsilon$ is sufficiently small, then we have

$$
\|f\|_{L_{t}^{r}\left([0, T], W_{x}^{1, p}\right)} \leq K_{1} \epsilon \text { for all admissible pairs }(r, p) .
$$

Consider $Z_{1}$ of the form (9.26). Set:

$$
G_{\mu \nu}^{0}=G_{\mu \nu}\left(\|f\|_{2}^{2}\right) \text { for }\|f\|_{2}^{2}=0 ; \lambda_{j}^{0}=\lambda_{j}\left(\omega_{0}\right)
$$

Then we have (with finite sums and with the derivative in the variable $\|f\|_{2}^{2}$ performed w.r.t. the $\|f\|_{2}^{2}$ arguments explicitly emphasized in Theorem 9.1)

$$
\begin{aligned}
& \mathrm{i} \dot{f}-\mathcal{H} f-2\left(\partial_{\|f\|_{2}^{2}} H\right) P_{c}\left(\omega_{0}\right) \sigma_{3} f=\sum_{\left|\lambda^{0} \cdot(\nu-\mu)\right|>\omega_{0}} z^{\mu} \bar{z}^{\nu} G_{\mu \nu}^{0} \\
& +\sum_{\left|\lambda^{0} \cdot(\nu-\mu)\right|>\omega_{0}} z^{\mu} \bar{z}^{\nu}\left(G_{\mu \nu}-G_{\mu \nu}^{0}\right)+\sigma_{3} \sigma_{1} \nabla_{f} \mathcal{R}-2\left(\partial_{\|f\|_{2}^{2}} \mathcal{R}\right) P_{c}\left(\omega_{0}\right) \sigma_{3} f .
\end{aligned}
$$


The proof of Proposition 10.2 is standard and is an easier version of the arguments in $\S 4$ in [CM]. The dominating term in the rhs of (10.8) is the one on the first line, with contribution to $f$ bounded by $C\left(C_{2}\right) \epsilon$ by the endpoint Strichartz estimate and by (10.5) (we recall that the third term in the lhs, in part becomes a phase through an integrating factor, in part goes on the rhs: see $[\mathrm{CM}]$; this trick is due to [BP2]). Notice also, that Theorem 10.1 implies by the arguments on pp. $67-68$ in $[\mathrm{CM}]$

$$
\lim _{t \rightarrow+\infty}\left\|e^{\mathrm{i} \theta(t) \sigma_{3}} f(t)-e^{\mathrm{i} t \Delta \sigma_{3}} f_{+}\right\|_{H^{1}}=0
$$

for a $f_{+} \in H^{1}$ with $\left\|f_{+}\right\|_{H^{1}} \leq C \epsilon$ and for $\theta(t)=t \omega_{0}+2 \int_{0}^{t}\left(\partial_{\|f\|_{2}^{2}} H\right)\left(t^{\prime}\right) d t^{\prime}$. We claim that $\theta(t)=\vartheta(t)-\vartheta(0)$. This claim, Theorem 9.1, Theorem 10.1 and (10.9) imply Theorem 8.1. To prove the claim we substitute the last system of coordinates in $(3.21)$ to obtain

$$
\text { i } \dot{f}-\mathcal{H} f-\left(\dot{\vartheta}-\omega_{0}\right) P_{c}\left(\omega_{0}\right) \sigma_{3} f=G
$$

where $G$ is a functional with values in $\in C\left(\mathbb{R}, L_{x}^{1}\right)$. The two equations are equivalent. This implies $G=\operatorname{rhs}(10.8)$ and $\dot{\vartheta}-\omega_{0}=2 \partial_{\|f\|_{2}^{2}} H$. This yields the claim $\theta(t)=\vartheta(t)-\vartheta(0)$.

Step (ii) in the proof of Theorem 10.1 consists in introducing the variable

$$
g=f+\sum_{\left|\lambda^{0} \cdot(\mu-\nu)\right|>\omega_{0}} z^{\mu} \bar{z}^{\nu} R_{\mathcal{H}}^{+}\left(\lambda^{0} \cdot(\mu-\nu)\right) G_{\mu \nu}^{0} .
$$

Substituting the new variable $g$ in (10.8), the first line on the rhs of (10.8) cancels out. By an easier version of Lemma $4.3[\mathrm{CM}]$ we have:

Lemma 10.3. For $\epsilon$ sufficiently small and for $C_{0}=C_{0}(\mathcal{H})$ a fixed constant, we have

$$
\|g\|_{L_{t}^{2} L_{x}^{2,-S}} \leq C_{0} \epsilon+O\left(\epsilon^{2}\right)
$$

As in $[\mathrm{CM}]$, the part of $f$ which couples nontrivially with $z$ comes from the polynomial in $z$ contained in (10.11). $g$ and $z$ are decoupled.

\subsection{The Fermi golden rule}

We proceed as in the related parts in $[\mathrm{BC}, \mathrm{CM}]$. The only difference with $[\mathrm{CM}]$ is that the preparatory work in Theorem 9.1 makes transparent the positive semidefiniteness of the crucial coefficients.

Set $R_{\mu \nu}^{+}=R_{\mathcal{H}}^{+}\left(\lambda^{0} \cdot(\mu-\nu)\right)$. We will have $\lambda_{j}^{0}=\lambda_{j}\left(\omega_{0}\right)$ and $\lambda_{j}=\lambda_{j}\left(\|f\|_{2}^{2}\right)$ as in Section 9.2. $\left|\lambda_{j}^{0}-\lambda_{j}\right| \lesssim C_{1}^{2} \epsilon^{2}$ by (10.4), so in the sequel we can assume that $\lambda^{0}$ satisfies the same inequalities of $\lambda$. We substitute (10.11) in $\mathrm{i} \dot{z}_{j}=\frac{\partial}{\partial \bar{z}_{j}} H^{(r)}$ 
obtaining

$$
\begin{aligned}
& \mathrm{i} \dot{z}_{j}=\partial_{\bar{z}_{j}}\left(H_{2}+Z_{0}\right)+\sum_{|\lambda \cdot(\mu-\nu)|>\omega_{0}} \nu_{j} \frac{z^{\mu} \bar{z}^{\nu}}{\bar{z}_{j}}\left\langle g, \sigma_{1} \sigma_{3} G_{\mu \nu}\right\rangle+\partial_{\bar{z}_{j}} \mathcal{R} \\
& -\sum_{\substack{|\lambda \cdot(\alpha-\beta)|>\omega_{0} \\
|\lambda \cdot(\mu-\nu)|>\omega_{0}}} \nu_{j} \frac{z^{\mu+\alpha} \bar{z}^{\nu+\beta}}{\bar{z}_{j}}\left\langle R_{\alpha \beta}^{+} G_{\alpha \beta}^{0}, \sigma_{1} \sigma_{3} G_{\mu \nu}\right\rangle .
\end{aligned}
$$

We rewrite this as

$$
\begin{aligned}
& \mathrm{i} \dot{z}_{j}=\partial_{\bar{z}_{j}}\left(H_{2}+Z_{0}\right)+\mathcal{E}_{j}
\end{aligned}
$$

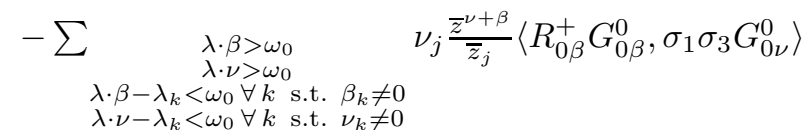

$$
\begin{aligned}
& -\sum_{\begin{array}{c}
\lambda \cdot \alpha>\omega_{0} \\
\lambda \cdot \nu>\omega_{0} \\
\lambda \cdot \alpha-\lambda_{k}<\omega_{0} \forall k \text { s.t. } \alpha_{k} \neq 0 \\
\lambda \cdot \nu-\lambda_{k}<\omega_{0} \forall k \text { s.t. } \nu_{k} \neq 0
\end{array}} \nu_{j} \frac{z^{\alpha} \bar{z}^{\nu}}{z_{j}}\left\langle R_{\alpha 0}^{+} G_{\alpha 0}^{0}, \sigma_{1} \sigma_{3} G_{0 \nu}^{0}\right\rangle .
\end{aligned}
$$

Here the elements in (10.15) will be eliminated through a new change of variables. $\mathcal{E}_{j}$ is a reminder term defined by

$$
\mathcal{E}_{j}:=\operatorname{rhs}(10.13)-(10.15)-(10.16)
$$

Set

$$
\begin{aligned}
& \zeta_{j}=z_{j}-\sum_{\substack{\lambda \cdot \beta>\omega_{0}, \lambda \cdot \nu>\omega_{0} \\
\lambda \cdot \beta-\lambda k<\omega_{0} \forall k \text { s.t. } \beta_{k} \neq 0 \\
\lambda \cdot \nu-\lambda_{k}<\omega_{0} \forall k \text { s.t. } \nu_{k} \neq 0}} \frac{\nu_{j}}{\lambda^{0} \cdot(\beta+\nu)} \frac{\bar{z}^{\nu+\beta}}{\bar{z}_{j}}\left\langle R_{0 \beta}^{+} G_{0 \beta}^{0}, \sigma_{1} \sigma_{3} G_{0 \nu}^{0}\right\rangle \\
& +\sum_{\begin{array}{c}
\lambda \cdot \alpha>\omega_{0}, \lambda \cdot \nu>\omega_{0} \\
\lambda^{0} \cdot \alpha \neq \lambda^{0} \cdot \nu \\
\lambda \cdot \alpha-\lambda_{k}<\omega_{0} \forall k \text { s.t. } \alpha_{k} \neq 0 \\
\lambda \cdot \nu-\lambda_{k}<\omega_{0} \forall k \text { s.t. } \nu_{k} \neq 0
\end{array}} \frac{\nu_{j}}{\lambda^{0} \cdot(\alpha-\nu)} \frac{z^{\alpha} \bar{z}^{\nu}}{\bar{z}_{j}}\left\langle R_{\alpha 0}^{+} G_{\alpha 0}^{0}, \sigma_{1} \sigma_{3} G_{0 \nu}^{0}\right\rangle
\end{aligned}
$$

Notice that in (10.17), by $\lambda \cdot \nu>\omega_{0}$, we have $|\nu|>1$. Then by (10.5)

$$
\begin{aligned}
& \|\zeta-z\|_{L_{t}^{2}} \leq C \epsilon \sum_{\substack{\lambda \cdot \alpha>\omega_{0} \\
\lambda \cdot \alpha-\lambda_{k}<\omega_{0} \forall k \text { s.t. } \alpha_{k} \neq 0}}\left\|z^{\alpha}\right\|_{L_{t}^{2}} \leq C C_{2} M \epsilon^{2} \\
& \|\zeta-z\|_{L_{t}^{\infty}} \leq C^{3} \epsilon^{3}
\end{aligned}
$$

with $C$ the constant in (10.3) and $M$ the number of terms in the rhs. In the new variables (10.14) is of the form

$$
\begin{aligned}
& \mathrm{i} \dot{\zeta}_{j}=\partial_{\bar{\zeta}_{j}} H_{2}(\zeta, f)+\partial_{\bar{\zeta}_{j}} Z_{0}(\zeta, f)+\mathcal{D}_{j} \\
& -\sum_{\substack{\lambda \cdot \alpha-\alpha=\lambda^{0} \cdot \nu>\omega_{0} \\
\lambda \cdot \alpha \lambda_{k}<\omega_{0} \forall k \text { s.t. } \alpha_{k} \neq 0 \\
\lambda \cdot \nu-\lambda_{k}<\omega_{0} \forall k \text { s.t. } \nu_{k} \neq 0}} \nu_{j} \frac{\zeta^{\alpha} \bar{\zeta}^{\nu}}{\bar{\zeta}_{j}}\left\langle R_{\alpha 0}^{+} G_{\alpha 0}^{0}, \sigma_{1} \sigma_{3} G_{0 \nu}^{0}\right\rangle .
\end{aligned}
$$


From these equations by $\sum_{j} \lambda_{j}^{0}\left(\bar{\zeta}_{j} \partial_{\bar{\zeta}_{j}}\left(H_{2}+Z_{0}\right)-\zeta_{j} \partial_{\zeta_{j}}\left(H_{2}+Z_{0}\right)\right)=0$ we get

$$
\begin{aligned}
& \partial_{t} \sum_{j=1}^{m} \lambda_{j}^{0}\left|\zeta_{j}\right|^{2}=2 \sum_{j=1}^{m} \lambda_{j}^{0} \operatorname{Im}\left(\mathcal{D}_{j} \bar{\zeta}_{j}\right)- \\
& -2 \sum_{\substack{\lambda^{0} \cdot \alpha=\lambda^{0} \cdot \nu>\omega_{0} \\
\lambda \cdot \alpha-\lambda_{k}<\omega_{0} \forall k \text { s.t. } \alpha_{k} \neq 0 \\
\lambda \cdot \nu-\lambda_{k}<\omega_{0} \forall k \text { s.t. } \nu_{k} \neq 0}} \lambda^{0} \cdot \nu \operatorname{Im}\left(\zeta^{\alpha} \bar{\zeta}^{\nu}\left\langle R_{\alpha 0}^{+} G_{\alpha 0}^{0}, \sigma_{1} \sigma_{3} G_{0 \nu}^{0}\right\rangle\right) .
\end{aligned}
$$

We have the following lemma, whose proof (we skip) is similar to Appendix B $[\mathrm{BC}]$.

Lemma 10.4. Assume inequalities (10.5). Then for a fixed constant $c_{0}$ we have

$$
\sum_{j}\left\|\mathcal{D}_{j} \bar{\zeta}_{j}\right\|_{L^{1}[0, T]} \leq\left(1+C_{2}\right) c_{0} \epsilon^{2}
$$

For the sum in the second line of (10.20) we get

$$
\begin{aligned}
& 2 \sum_{r>\omega_{0}} r \operatorname{Im}\left\langle R_{\mathcal{H}}^{+}(r) \sum_{\lambda^{0} \cdot \alpha=r} \zeta^{\alpha} G_{\alpha 0}^{0}, \sigma_{1} \sigma_{3} \sum_{\lambda^{0} \cdot \nu=r} \bar{\zeta}^{\nu} G_{0 \nu}^{0}\right\rangle= \\
& 2 \sum_{r>\omega_{0}} r \operatorname{Im}\left\langle R_{\mathcal{H}}^{+}(r) \sum_{\lambda^{0} \cdot \alpha=r} \zeta^{\alpha} G_{\alpha 0}^{0}, \sigma_{3} \overline{\sum_{\lambda^{0} \cdot \alpha=r} \zeta^{\alpha} G_{\alpha 0}^{0}}\right\rangle,
\end{aligned}
$$

where we have used $G_{\mu \nu}^{0}=-\sigma_{1} \bar{G}_{\nu \mu}^{0}$. Then we have the key structural result of this paper.

Lemma 10.5. We have rhs $(10.22) \geq 0$.

Proof. First of all, it is not restrictive to assume $G_{\alpha 0}^{0}=P_{c}\left(\omega_{0}\right) G_{\alpha 0}^{0}$. We have $G_{\alpha 0}^{0} \in \mathcal{S}\left(\mathbb{R}^{3}, \mathbb{C}^{2}\right)$ for all $\alpha$. For $W(\omega)=\lim _{t \rightarrow \infty} e^{-i t \mathcal{H}_{\omega}} e^{i t \sigma_{3}(-\Delta+\omega)}$, there exist $F_{\alpha} \in W^{k, p}\left(\mathbb{R}^{3}, \mathbb{C}^{2}\right)$ for all $k \in \mathbb{R}$ and $p \geq 1$ with $G_{\alpha 0}^{0}=W\left(\omega_{0}\right) F_{\alpha}$, [Cu1]. By standard theory, $R_{\mathcal{H}}^{+}(r) G_{\alpha 0}^{0} \in L^{2,-s}\left(\mathbb{R}^{3}, \mathbb{C}^{2}\right)$ for any $s>1 / 2$ and $r>\omega_{0}$. Let $\mathbf{G}=\sum_{\lambda^{0} \cdot \alpha=r} \zeta^{\alpha} G_{\alpha 0}^{0}$ and $\mathbf{F}=\sum_{\lambda^{0} \cdot \alpha=r} \zeta^{\alpha} F_{\alpha}$. Let ${ }^{t} \mathbf{F}=\left(\mathbf{F}_{1}, \mathbf{F}_{2}\right)$. Then, see Lemma $4.1[\mathrm{Cu} 2]$,

$$
\begin{aligned}
& \operatorname{Im}\left\langle R_{\mathcal{H}}^{+}(r) \mathbf{G}, \sigma_{3} \overline{\mathbf{G}}\right\rangle=\lim _{\varepsilon \searrow 0} \operatorname{Im}\left\langle R_{\mathcal{H}}(r+\mathrm{i} \varepsilon) \mathbf{G}, \sigma_{3} \overline{\mathbf{G}}\right\rangle \\
& =\lim _{\varepsilon \searrow 0} \operatorname{Im}\left\langle R_{\sigma_{3}\left(-\Delta+\omega_{0}\right)}(r+\mathrm{i} \varepsilon) \mathbf{F}, \sigma_{3} \overline{\mathbf{F}}\right\rangle \\
& =\lim _{\varepsilon \searrow 0} \operatorname{Im}\left\langle R_{-\Delta}\left(r-\omega_{0}+\mathrm{i} \varepsilon\right) \mathbf{F}_{1}, \overline{\mathbf{F}_{1}}\right\rangle \\
& =\lim _{\varepsilon \searrow 0} \int_{\mathbb{R}^{3}} \frac{\varepsilon}{\left(\xi^{2}-\left(r-\omega_{0}\right)\right)^{2}+\varepsilon^{2}}\left|\widehat{\mathbf{F}}_{1}(\xi)\right|^{2} d \xi \geq 0 .
\end{aligned}
$$

Now we will assume the following hypothesis. 
(H11) We assume that for some fixed constants for any vector $\zeta \in \mathbb{C}^{n}$ we have:

$$
\begin{aligned}
& \sum_{\substack{\lambda^{0} \cdot \alpha=\lambda^{0} \cdot \nu>\omega_{0} \\
\lambda \cdot \alpha-\lambda_{k}<\omega_{0} \forall k \text { s.t. } \alpha_{k} \neq 0 \\
\lambda \cdot \nu-\lambda_{k}<\omega_{0} \forall k \text { s.t. } \nu_{k} \neq 0}} \lambda^{0} \cdot \nu \operatorname{Im}\left(\zeta^{\alpha} \bar{\zeta}^{\nu}\left\langle R_{\alpha 0}^{+} G_{\alpha 0}^{0}, \sigma_{1} \sigma_{3} G_{0 \nu}^{0}\right\rangle\right) \\
& \approx \sum_{\substack{\lambda^{0} \cdot \alpha>\omega_{0} \\
\lambda^{0} \cdot \alpha-\lambda_{k}^{0}<\omega_{0} \forall k \text { s.t. } \alpha_{k} \neq 0}}\left|\zeta^{\alpha}\right|^{2} .
\end{aligned}
$$

By (H11) we have

$$
\begin{gathered}
2 \sum_{j} \lambda_{j}^{0} \operatorname{Im}\left(\mathcal{D}_{j} \bar{\zeta}_{j}\right) \gtrsim \partial_{t} \sum_{j} \lambda_{j}^{0}\left|\zeta_{j}\right|^{2}+ \\
\sum_{\substack{\lambda^{0} \cdot \alpha>\omega_{0} \\
\lambda^{0} \cdot \alpha-\lambda_{k}^{0}<\omega_{0} \forall k \text { s.t. } \alpha \\
k \neq 0}}\left|\zeta^{\alpha}\right|^{2}
\end{gathered}
$$

Then, for $t \in[0, T]$ and assuming Lemma 10.4 we have

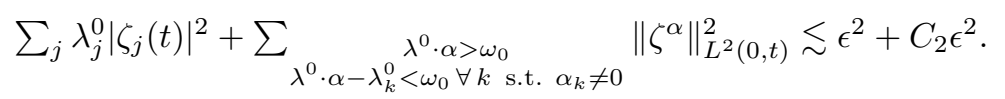

By (10.18) this implies $\left\|z^{\alpha}\right\|_{L^{2}(0, t)}^{2} \lesssim \epsilon^{2}+C_{2} \epsilon^{2}$ for all the above multi indexes. So, from $\left\|z^{\alpha}\right\|_{L^{2}(0, t)}^{2} \lesssim C_{2}^{2} \epsilon^{2}$ we conclude $\left\|z^{\alpha}\right\|_{L^{2}(0, t)}^{2} \lesssim C_{2} \epsilon^{2}$. This means that we can take $C_{2} \approx 1$. This yields Theorem 10.1.

Remark 10.6. Notice that by $r>\omega_{0},(10.24)$ appears generic. We do not try to prove this point. It should not be hard, see for example the genericity result Proposition $2.2[\mathrm{BC}]$.

Remark 10.7. In general we expect Hypothesis (H11), or higher order versions, to hold. Specifically, if at some step of the normal form argument (H11) fails because some of the inequalities as in Lemma 10.5 is an equality, one can continue the normal form procedure and obtain some steps later a new version of (H11). This will yield an analogue of Theorem 10.1, with 10.2 replaced by a similar but weaker inequality. We could have stated (H11) and proved Theorem 10.1 in this more general form, but this would have complicated further the presentation.

Remark 10.8. If instead of ground states we consider standing waves with nodes, and if $\operatorname{dim} N_{g}\left(\mathcal{H}_{\omega}\right)=2$ with (3.10), if we assume (H1)-(H11) with (2.2) in (H5) replaced by $\frac{d}{d \omega}\left\|\phi_{\omega}\right\|_{L^{2}\left(\mathbb{R}^{3}\right)}^{2} \neq 0$, if we assume $\sigma\left(\mathcal{H}_{\omega}\right) \subset \mathbb{R}$, then by [Cu3] it is possible to prove that the hamiltonian $K$ in Lemma 8.2 has quadratic part

$$
K_{2}=\sum_{j=1}^{m} \gamma_{j} \lambda_{j}(\omega)\left|z_{j}\right|^{2}+\frac{1}{2}\left\langle\sigma_{3} \mathcal{H}_{\omega} f, \sigma_{1} f\right\rangle
$$

with $\gamma_{j}$ equal to either 1 or -1 and with at least one $\gamma_{j}=-1$ (in other words the energy has a saddle at $\phi_{\omega}$ in the surface formed by the $u$ with $\left.\|u\|_{L^{2}}=\left\|\phi_{\omega}\right\|_{L^{2}}\right)$. 
Then a simple elaboration of the proof of the present paper, along the lines of sections 3 or 4 in [Cu3], can be used to strengthen Theorem 3.2 [Cu3] showing that $\phi_{\omega}$ is orbitally unstable. Furthermore, following the argument in [Cu3], it can be shown that if a solution $u(t)$ remains close to ground states as $t \nearrow+\infty$ (resp. $t \searrow-\infty$ ), it actually scatters to ground states, that is (2.3) and (2.5) with the plus (resp. minus) sign.

\section{References}

[BC] D.Bambusi, S.Cuccagna, On dispersion of small energy solutions of the nonlinear Klein Gordon equation with a potential, http://www.dismi.unimore.it/Members/scuccagna/pubblicazioni/ NLKGsmall11.pdf/view.

[BP1] V.Buslaev, G.Perelman, Scattering for the nonlinear Schrödinger equation: states close to a soliton, St. Petersburg Math.J., 4 (1993), pp. $1111-1142$.

[BP2] V.Buslaev, G.Perelman, On the stability of solitary waves for nonlinear Schrödinger equations, Nonlinear evolution equations, editor N.N. Uraltseva, Transl. Ser. 2, 164, Amer. Math. Soc., pp. 75-98, Amer. Math. Soc., Providence (1995).

[BS] V.S.Buslaev, C.Sulem, On the asymptotic stability of solitary waves of Nonlinear Schrödinger equations, Ann. Inst. H. Poincaré. An. Nonlin., 20 (2003), pp. 419-475.

[CL] T.Cazenave, P.L.Lions, Orbital stability of standing waves for nonlinear Schrödinger equations, Comm. Math. Phys. 85 (1982), 549-561.

[Cu1] S.Cuccagna, Stabilization of solutions to nonlinear Schrödinger equations, Comm. Pure App. Math. 54 (2001), pp. 1110-1145, erratum Comm. Pure Appl. Math. 58 (2005), p. 147.

[Cu2] S.Cuccagna, On asymptotic stability of ground states of NLS, Rev. Math. Phys. 15 (2003), pp. 877-903.

[Cu3] S.Cuccagna, On instability of excited states of the nonlinear Schrödinger equation,Physica D, 238 (2009), pp. 38-54.

[CM] S.Cuccagna, T.Mizumachi, On asymptotic stability in energy space of ground states for Nonlinear Schrödinger equations, Comm. Math. Phys., 284 (2008), pp. 51-87.

[CPV] S.Cuccagna, D.Pelinovsky, V.Vougalter, Spectra of positive and negative energies in the linearization of the NLS problem, Comm. Pure Appl. Math. 58 (2005), pp. 1-29. 
[CT] S.Cuccagna, M.Tarulli, On asymptotic stability of standing waves of discrete Schrödinger equation in Z , SIAM J. Math. Anal. 41, (2009), pp. $861-885$

[Gz] Zhou Gang, Perturbation Expansion and N-th Order Fermi Golden Rule of the Nonlinear Schrödinger Equations, J. Math. Phys., 48( 2007), p. 053509

[GS] Zhou Gang, I.M.Sigal, Relaxation of Solitons in Nonlinear Schrödinger Equations with Potential, Advances in Math., 216 (2007), pp. 443-490.

[GW1] Zhou Gang, M.I.Weinstein, Dynamics of Nonlinear Schrödinger/GrossPitaeskii Equations; Mass transfer in Systems with Solitons and Degenerate Neutral Modes, Anal. PDE 1 (2008), pp. 267-322.

[GW2] Zhou Gang, M.I.Weinstein, Equipartition of Energy in Nonlinear Schrödinger/Gross-Pitaeskii Equations, in preparation.

[GSS1] M.Grillakis, J.Shatah, W.Strauss, Stability of solitary waves in the presence of symmetries, I, Jour. Funct. An. 74 (1987), pp.160-197.

[GSS2] M.Grillakis, J.Shatah, W.Strauss, Stability of solitary waves in the presence of symmetries, II, Jour. Funct. An. 94 (1990), pp. 308-348.

[GNT] S.Gustafson, K.Nakanishi, T.P.Tsai, Asymptotic Stability and Completeness in the Energy Space for Nonlinear Schrödinger Equations with Small Solitary Waves, Int. Math. Res. Notices 66 (2004), pp. 35593584 .

[JSS] J.L.Journe, A.Soffer, C.D.Sogge, Decay estimates for Schrodinger operators , Comm.P. Appl. Mat. 44 (1991), pp. 573-604.

[K] T.Kato, Wave operators and similarity for some non-selfadjoint operators, Math. Annalen, 162 (1966), pp. 258-269.

[KS] J.Krieger, W.Schlag, Stable manifolds for all monic supercritical focusing nonlinear Schrödinger equations in one dimension, J. Amer. Math. Soc., 19 (2006), pp. 815-920.

[HW] A.Hoffman, C. E. Wayne, Asymptotic two-soliton solutions in the FermiPasta-Ulam model, J. Dynam. Differential Equations 21 (2009), pp. 343-351.

[MM1] Y.Martel, F.Merle, Asymptotic stability of solitons of the gKdV equations with general nonlinearity, Math. Ann. 341 (2008), pp. 391-427.

[MM2] Y.Martel, F.Merle, Stability of two soliton collision for nonintegrable gKdV equations, Comm. Math. Phys. 286 (2009), pp. 39-79. 
[MMT] Y.Martel, F.Merle, T.P.Tsai, Stability in $H^{1}$ of the sum of $K$ solitary waves for some nonlinear Schrdinger equations, Duke Math. J. 133 (2006), pp. 405-466.

[MR] F.Merle, P.Raphael, On a sharp lower bound on the blow-up rate for the $L^{2}$ critical nonlinear Schrödinger equation, J. Amer. Math. Soc. 19 (2006), pp. 37-90.

[M1] T.Mizumachi, Asymptotic stability of small solitons to $1 D$ NLS with potential, Jour. of Math. Kyoto University, 48 (2008), pp. 471-497.

[M2] T.Mizumachi, Asymptotic stability of small solitons for $2 D$ Nonlinear Schrödinger equations with potential, Jour. of Math. Kyoto University, 43 (2007), pp. 599-620.

[M3] T.Mizumachi, Asymptotic stability of $N$-solitons of the FPU lattices, arXiv:0906.1320v1.

[PW] R.L.Pego, M.I.Weinstein, Asymptotic stability of solitary waves Comm. Math. Phys. 164 (1994), pp. 305-349.

[PiW] C.A.Pillet, C.E.Wayne, Invariant manifolds for a class of dispersive, Hamiltonian partial differential equations J. Diff. Eq. 141 (1997), pp. 310-326.

[S] W.Schlag, Stable manifolds for an orbitally unstable NLS, Ann. of Math. 169 (2009), pp. 139-227

[Si] I.M.Sigal, Nonlinear wave and Schrödinger equations. I. Instability of periodic and quasi- periodic solutions, Comm. Math. Phys. 153 (1993), pp. 297-320.

[ShS] J.Shatah, W.Strauss Instability of nonlinear bound states, Comm. Math. Phys. 100 (1985), pp. 173-190

[SW1] A.Soffer, M.I.Weinstein, Multichannel nonlinear scattering for nonintegrable equations, Comm. Math. Phys., 133 (1990), pp. 116-146

[SW2] A.Soffer, M.I.Weinstein, Multichannel nonlinear scattering II. The case of anisotropic potentials and data, J. Diff. Eq., 98 (1992), pp. 376-390.

[SW3] A.Soffer, M.I.Weinstein, Resonances, radiation damping and instability in Hamiltonian nonlinear wave equations , Invent. Math., 136 (1999), pp. 9-74.

[SW4] A.Soffer, M.I.Weinstein, Selection of the ground state for nonlinear Schrödinger equations, Rev. Math. Phys. 16 (2004), pp. 977-1071.

[St] W.Strauss, Nonlinear wave equations, CBMS Regional Conf. Ser. Mat. AMS 76 (1989). 
[T] T.P.Tsai, Asymptotic dynamics of nonlinear Schrödinger equations with many bound states, J. Diff. Eq. 192 (2003), pp. 225-282.

[TY1] T.P.Tsai, H.T.Yau, Asymptotic dynamics of nonlinear Schrödinger equations: resonance dominated and radiation dominated solutions, Comm. Pure Appl. Math. 55 (2002), pp. 153-216.

[TY2] T.P.Tsai, H.T.Yau, Relaxation of excited states in nonlinear Schrödinger equations, Int. Math. Res. Not. 31 (2002), pp. 1629-1673.

[TY3] T.P.Tsai, H.T.Yau, Classification of asymptotic profiles for nonlinear Schrödinger equations with small initial data, Adv. Theor. Math. Phys. 6 (2002), pp. 107-139.

[W1] M.I.Weinstein, Lyapunov stability of ground states of nonlinear dispersive equations, Comm. Pure Appl. Math. 39 (1986), pp. 51-68.

[W2] M.I.Weinstein, Modulation stability of ground states of nonlinear Schrödinger equations, Siam J. Math. Anal. 16 (1985), pp. 472-491.

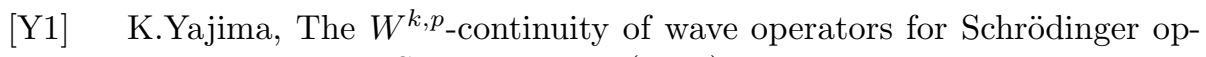
erators, J. Math. Soc. Japan, 47 (1995), pp. 551-581.

[Y2] K.Yajima, The $W^{k, p}$-continuity of wave operators for Schrödinger operators III., J. Math. Sci. Univ. Tokyo, 2 (1995), pp. 311-346.

DISMI University of Modena and Reggio Emilia, Via Amendola 2, Pad. Morselli, Reggio Emilia 42122, Italy.

E-mail Address: cuccagna.scipio@unimore.it 